\title{
HectD1 Controls Hematopoietic Stem Cell Regeneration by Coordinating Ribosome Assembly and Protein Synthesis
}

Kaosheng Lv, ${ }^{1,2}$ Chujie Gong, ${ }^{1,2}$ Charles Antony, ${ }^{3}$ Xu Han, ${ }^{1,2}$ Jian-Gang Ren, ${ }^{1,2}$ Ryan Donaghy, ${ }^{1,2}$ Ying Cheng, ${ }^{1,2,4}$ Simone Pellegrino, ${ }^{5,6,7}$ Alan J. Warren, ${ }^{5,6,7}$ Vikram R. Paralkar, ${ }^{3}$ and Wei Tong $1,2,8, *$

1 Division of Hematology, Children's Hospital of Philadelphia, Philadelphia, PA 19104

2 Division of Pediatrics, Perelman School of Medicine at the University of Pennsylvania, Philadelphia, PA 19104

3 Division of Hematology-Oncology, Department of Medicine, Perelman School of Medicine at the University of Pennsylvania, Philadelphia, PA 19104

4 Present address: Center for Mitochondrial Biology and Medicine, The Key Laboratory of Biomedical Information Engineering of Ministry of Education, School of Life Science and Technology, Xi'an Jiaotong University, Xi'an, China. 710049

5 Cambridge Institute for Medical Research, Cambridge, United Kingdom

6 Department of Haematology, University of Cambridge, Cambridge, United Kingdom

7 Wellcome Trust-Medical Research Council Stem Cell Institute, University of Cambridge, Cambridge, United Kingdom

8 Lead Contact

*Correspondence author: $\quad$ tongw@chop.edu

\section{Keywords}

Hematopoietic stem cells (HSCs), ubiquitin, signaling, ribosome biogenesis, protein synthesis, ribosome assembly.

Running title: HectD1 controls ribosome assembly in HSCs 


\section{Summary}

Impaired ribosome function is the underlying etiology in a group of bone marrow failure syndromes called ribosomopathies. However, how ribosomes are regulated remains poorly understood, as are approaches to restore hematopoietic stem cell (HSC) function attributable to defective ribosome biogenesis. Here we reveal a role for the E3 ubiquitin ligase HectD1 in regulating HSC function via ribosome assembly and protein translation. Hectdl-deficient HSCs exhibit a striking defect in transplantation ability and ex vivo maintenance, concomitant with a reduced protein synthesis and growth rate under stress conditions. Mechanistically, HectD1 ubiquitinates and degrades ZNF622, an assembly factor for the ribosomal 60S subunit. HectD1 loss led to an accumulation of ZNF622 and the anti-association factor eIF6 on the 60S, resulting in 60S/40S joining defects. Importantly, Znf622 depletion in Hectdl-deficient HSCs restored ribosomal subunit joining, protein synthesis, and HSC reconstitution capacity. These findings highlight the importance of ubiquitin-coordinated ribosome assembly in HSC regeneration. 


\section{Introduction}

At the steady-state, protein synthesis rate is low in adult hematopoietic stem cells (HSCs) as compared with committed progenitors or differentiated cells (Signer et al., 2014). Tightlyregulated protein synthesis rate is critical for HSC maintenance and function (Signer et al., 2014; Signer et al., 2016). HSC expansion under regenerative or stress conditions demands increased ribosome biogenesis and protein synthesis. Appropriate ribosome biogenesis and assembly ensures the translation efficiency and fidelity of proteins, which are important for normal development as well as prevention of cancer. Mutations in ribosomal proteins and gene products affecting ribosome biogenesis and protein synthesis are associated with human diseases marked by hematopoietic dysfunction (Sulima et al., 2017). However, how ribosome assembly is regulated in HSCs remains poorly understood, as is its contribution to hematopoietic diseases.

The biogenesis of the two ribosomal subunits $40 \mathrm{~S}$ and $60 \mathrm{~S}$ occurs largely in the nucleus; upon nuclear export, they separately undergo final stages of maturation in the cytoplasm that are regulated by distinct assembly factors and incorporation of the last few cytoplasmic ribosome proteins (de la Cruz et al., 2015). These assembly factors proofread and protect key functional sites on the ribosome and prevent premature joining of $60 \mathrm{~S}$ and $40 \mathrm{~S}$ subunits, to ensure regulated formation of functional 80S monosomes and appropriate translation (Klinge and Woolford, 2019). "Ribosomopathies" are characterized by a group of inherited bone marrow failure (BMF) syndromes with impaired ribosome function. Individuals with "ribosomopathies" are deficient in HSCs or specific lineages of blood formation, and yet are predisposed to elevated leukemia and cancer risks (Ruggero and Shimamura, 2014). One such example is Shwachman-Diamond syndrome (SDS), that is etiologically linked to ribosome dysfunction arising from mutations of ribosome assembly factors (Warren, 2018; Woloszynek et al., 2004). Germline mutations in three different genes (SBDS, DNAJC21, and EFL1) involved in the 60S maturation and assembly all 
cause SDS (Boocock et al., 2003; Dhanraj et al., 2017; Tan et al., 2019; Tummala et al., 2016; Woloszynek et al., 2004), implying that HSCs are especially sensitive to perturbations in ribosome assembly. These mutations result in ribosomal subunit joining defects and decreased protein synthesis rate (Finch et al., 2011; Menne et al., 2007; Tan et al., 2019; Wong et al., 2011). The question of how ribosomal abnormalities cause marrow failure and cancer predisposition is therefore of fundamental biological and clinical interest. However, how assembly factors themselves are regulated and their impact on hematopoietic regeneration remain poorly understood.

HectD1, a member of HECT domain E3 ligases, plays an indispensable role in early embryogenesis. The HECT domain of HectD1 catalyzes the ubiquitination of its substrates to modulate protein stability, protein-protein interaction, and cellular localization. HectD1 has been reported to regulate various biological processes, including signaling transduction, gene transcription, development, and lipid homeostasis (Aleidi et al., 2018; Li et al., 2015; Sarkar and Zohn, 2012; Sugrue et al., 2019; Tran et al., 2013). Here, we identify HectD1 as a critical determinant of HSC function via its direct ubiquitination of ribosomal assembly factor. This discovery provides an important new facet to our understanding of ubiquitin-coordinated ribosomal assembly in stem cell biology.

\section{Results}

\section{Generation of a conditional Hectdl knockout mice in hematopoietic cells.}

Hectdl germline knockout $(\mathrm{KO})$ leads to mouse embryonic lethality due to defects in neural tube closure and impaired placenta development (D'Alonzo et al., 2019; Sarkar et al., 2014; Zohn et al., 2007). To explore a potential role for HectD1 in hematopoiesis, we studied a conditional Hectdl knockout (cKO) mouse model (Figure S1A). The floxed alleles of Hectdl that target exon 3 $\left(H e c t d l^{f / f}\right)$ were crossed with Vav-cre transgenic mice, in which the Cre recombinase is under the 
control of the Vav promoter to allow pan-hematopoietic excision of Hectdl in all hematopoietic cells $\left(H e c t d l^{f l ;: V a v}\right)$. Deletion of exon 3 is predicted to generate an early stop codon in exon 4, thus producing a non-functional 50 amino acid truncated protein. To evaluate deletion efficiency, we plated bone marrow (BM) cells from Hectdl ${ }^{f l f}$ and Hectd ${ }^{f l f ; V a v}$ mice into semisolid methylcellulose culture and confirmed a nearly 100\% deletion efficiency by genotyping individual colonies (Figure S1B). Quantitative PCR analysis (qRT-PCR) demonstrated the specific targeting of exon 3 of the Hectdl gene (Figures S1C and S1D). Moreover, HectD1 protein was undetectable in Hectdl flf; Vav BM cells (Figure S1E). Together, these data suggest that Hectdl was efficiently and specifically deleted in all hematopoietic cells in our cKO mouse model.

\section{Hectdl deficiency impairs HSC repopulation capacity and decreases functional HSC}

\section{frequency}

To elucidate the impact of Hectdl loss on homeostatic hematopoiesis, we first investigated the hematological parameters in young adult mice. Complete blood count $(\mathrm{CBC})$ analysis and flow cytometry of different lineages of blood cells from Hectd $f^{f f f: V a v}$ mice showed largely normal blood counts compared with their Hectdl flf littermates (Figures S2A and S2B). Total BM cellularity and spleen weight were also indistinguishable from controls (Figures S2C and S2D). We then characterized the hematopoietic stem and progenitor cells (HSPCs) in the BM and spleen. Hectdl $I^{f f ; \text { Vav }}$ mice showed comparable HSPC frequencies and numbers to those of Hectdl ${ }^{f f f}$ controls (Figures S2E-S2H), ie, long-term stem cells (LT-HSCs, Lin $^{-S c a 1}{ }^{+} \mathrm{c}-\mathrm{Kit}^{+} \mathrm{Flk} 2^{-} \mathrm{CD} 150^{+} \mathrm{CD}^{-} 8^{-}$), short-term stem cells (ST-HSCs, Flk2-CD150CD48- ${ }^{-} \mathrm{LSK}$ ), various multipotent progenitors (MPPs) (Lv et al., 2017). Furthermore, we observed no difference in the frequency and number of committed progenitor cells in the BM by flow cytometry (Figures S2I and S2J) or functional progenitors by colony-forming-cell (CFC) assay (Figure S2K). Interestingly, the spleen of Hectd $I^{f f f \text { Vav }}$ mice displayed a significant increase in CMP and MEP progenitors (Figures S2L and 
$\mathrm{S} 2 \mathrm{M}$ ), and accordingly, more CFU-E and CFU-GM colonies as compared to their Hectdl ${ }^{f / f}$ littermates (Figure S2N). Moreover, the cell cycle status or apoptosis in HSCs and MPPs remained unchanged (Figures S2O-2Q). These observations indicate relatively normal hematopoiesis in young adult Hectdl $I^{f f ;: V a v}$ mice under homeostatic conditions.

To assess the consequences of Hectdl loss in HSC function in vivo, we performed competitive BM transplantation (BMT) assay by injecting $1 \times 10^{6}$ total BM cells from Hectdl ${ }^{f / f}$ or Hectdl $1^{f f ;}$; Vav donor mice (CD45.2) with an equal number of BM cells from competitor mice (CD45.1) into lethally irradiated recipients (CD45.1/CD45.2) (Figure 1A). Donor chimerism in the peripheral blood (PB) of the recipients was determined by flow cytometry every 4 weeks post-BMT. Of note, donor-derived cell percentages from Hectdl ${ }^{f l f \text { :Vav }}$ mice were considerably decreased compared with control mice as early as 4 weeks, and exhibited a progressive decline over time (Figures 1B and 1C), indicating a defect in LT-repopulating HSCs. Hectdl loss did not affect lineage distribution in the transplanted recipients (Figures 1D and S3A-S3D). Importantly, the percentages of donor-derived HSPC populations from Hectdl fl;:Vav mice were substantially decreased in comparison to Hectdl $\mathrm{I}^{\mathrm{flf}}$ controls (Figure1E), indicating a reduction in HSC reconstitution. To further examine the impact of Hectdl loss on HSC self-renewal, we performed secondary BMT by injecting $1 \times 10^{6}$ total $\mathrm{BM}$ cells from primary transplanted mice into secondary recipients. Defective reconstitution from Hectdl $I^{f f ;: V a v}$ mice was further exacerbated in secondary transplantation (Figure 1F). Thus, these data indicate that Hectdl flf;Vav mice have inferior longterm BM repopulating HSCs with reduced self-renewal.

To functionally quantify HSC frequency, we next employed limiting dilution BMT (Figure 1G). A graded dose of BM cells from Hectdl ${ }^{f f f}$ and Hectd1 ${ }^{\text {fl; } \text { Vav }}$ mice mixed with a fixed number of competitor cells were transplanted into lethally irradiated recipient mice. 16 weeks after BMT, 
mice with donor percentage higher than $1 \%$ were defined as "positive reconstitution" (Bersenev et al., 2008). We found that Hectdl ${ }^{f f ; \text { Vav }}$ mice harbored a 4-fold reduction in functional HSC frequencies in the BM by ELDA analysis (extreme limiting dilution analysis) (Figure $1 \mathrm{H})(\mathrm{Hu}$ and Smyth, 2009). Hectdl ${ }^{f f ;: V a v}$ BM cells exhibited consistently reduced donor chimerisms in the recipients transplanted at all doses of donor cells (Figures 1I and S3E-S3G). Since HectD1 was reported to be involved in protein secretion and cell migration (Duhamel et al., 2018), we asked if Hectdl loss could affect BM niche or in a cell extrinsic manner. Therefore, we performed reciprocal BMT by injecting BM cells from wildtype mice (CD45.1) into Hectdl ${ }^{f / f}$ or Hectdlff;:Vav mice as recipients (CD45.2) (Figure $\mathrm{S} 3 \mathrm{H})$. Donor chimerism as well as lineage distribution in the PB and HSPCs in the BM were comparable among recipients of both genotypes (Figures S3I-S3K). Taken together, our data provide strong evidence that Hectdl insufficiency reduces frequencies of functional HSCs in the BM.

\section{Hectd1 insufficiency diminishes HSC reconstituting activity in vivo and HSC growth ex vivo}

To further address if the decreased repopulation observed in total BM transplantation is due to intrinsic HSC properties, we purified LT-HSCs (LSK CD150 ${ }^{+} \mathrm{CD} 48^{-}$) from Hectdlfff and Hectdl flf:Vav mice by flow cytometric sorting and transplanted 100 HSCs into each lethally-irradiated recipient along with Sca1-depleted BM competitors (Figure 2A) (Balcerek et al., 2018). Our results demonstrated that Hectdl-deficient HSCs exhibited inferior reconstitution ability to that of control HSCs in vivo (Figure 2B). Importantly, donor-derived HSPCs in the BM of recipient mice transplanted with Hectdl-deficient HSCs were significantly reduced when compared with those from control HSCs (Figure 2C), indicating Hectdl insufficiency decreases intrinsic HSC activity.

We next analyzed the influence of HectD1 on the maintenance of HSC function upon ex vivo culture. Purified HSCs were cultured in media containing a combination of cytokines SCF, TPO, 
FLT3L and IL6 for 12 days. The resultant cultured cells from 100 HSCs were transplanted (Figure 2A). Our data revealed that Hectdl insufficiency failed to maintain HSCs ex vivo, which was accompanied by a marked reduction in reconstituting capacity $(9.0 \%$ cultured HSCs vs $57.8 \%$ fresh HSCs reconstitution at 16 weeks), while control HSCs showed comparable reconstitution after culture (92.0\% cultured HSCs vs $82.0 \%$ fresh HSCs) (Figure 2D). Moreover, examination of donor-derived HSPCs in the BM of recipient mice revealed that Hectdl-deficient HSCs completely lost their stem cell identity in culture (Figure 2E). Importantly, we found that Hectdldeficient HSCs had a visibly slower growth rate than control HSCs ex vivo as determined by enumeration of cell numbers, and this phenotype persisted irrespective of cytokines used in culture (Figures 2F-2I). Taken together, our data suggest that HectD1 is critical for HSC function in vivo and HSC growth ex vivo.

Since HectD1 is widely expressed in a range of hematopoietic cells, we asked if HectD1 is preferentially required for HSC function. To test this, we injected 500 purified HSCs (LSK $\mathrm{CD} 150^{+} \mathrm{CD}^{4} 8^{-}$) or $5000 \mathrm{MPPs} /$ hematopoietic progenitors (HPCs) (LSK CD150-CD48 ${ }^{+}$) (Oguro et al., 2013; Pietras et al., 2015; Wilson et al., 2008) from Hectd ${ }^{f l f}$ and Hectdl ${ }^{\text {fl;:Vav }}$ mice into each sub-lethally irradiated recipient and analyzed donor chimerism every week post-BMT (Figure 2J). Of note, our results showed that Hectdl deficiency markedly decreased HSC reconstitution ability, but not that of MPP/HPCs (Figures 2K, 2L and S3L, S3M), indicating that HectD1 plays a critical role in regulating HSC function.

\section{HectD1 interacts with, ubiquitinates and regulates the stability of ZNF622}

To study the mechanisms underlying the reduced growth of Hectdl-deficient HSPCs, we first evaluated known signaling pathways important for HSC function, ie, JAK-STAT, PI3K-AKT, MAPK-ERK, mTOR, GSK3 and $\beta$-catenin pathways (Figure S4A). However, none of these 
signaling molecules were altered, except for a significant reduction of phospho-RPS6 (pRPS6) in both freshly isolated and cultured Hectdl-deficient LSK cells (Figures 3A and S4B).

To facilitate downstream biochemical studies, we resorted to cytokine-dependent human progenitor cell line, TF-1 cells. Using two different shRNAs to stably deplete HECTD1 in human TF-1 cells, in comparison to Luciferase (Luc) control (Figure 3B), we observed a significant growth retardation in the presence of TPO or GM-CSF upon HECTD1 knockdown (Figures 3C and 3D), consistent with findings from HSPCs (Figures 2F-2I). TF-1 cells also recapitulated the signaling defects observed in primary LSK cells, which is a reduction in RPS6 phosphorylation but not any other pathways we examined (Figures S4C-S4E). Thus, TF-1 cells appear to be a reliable and robust cell system for us to further dissect HectD1 functions.

Intriguingly, the reduction of pRPS6 seemed to be independent of mTOR pathway since p-mTOR, p4E-BP1, pS6K1 and a S6K1 substrate pZRF (Barilari et al., 2017) were not changed (Figures 3A and S4B). This led us to hypothesize that HectD1 might directly ubiquitinate RPS6 or regulate S6K1-RPS6 interaction as K63-ubiquitination is known to affect protein complex formation (Shembade and Harhaj, 2015). However, neither RPS6 ubiquitination nor the S6K1-RPS6 interaction was impacted by HectD1 E3 dead mutant C2579G (Mut) (Sarkar and Zohn, 2012) or HECTD1 knockdown (Figures S4F-S4H), implying that HectD1 indirectly affects RPS6 phosphorylation. The phospho-RPS6 has been shown to control the RiBi- (ribosome biogenesis) gene transcriptional program (Chauvin et al., 2014). We found that Hectdl-deficient HSCs did not exhibit changes in either total RNA level that is predominantly rRNAs or the mRNA level of the RiBi genes relatively to that of control cells (Figures S4I and S4J). 
In an attempt to explore the molecular mechanism underlying the important role for Hectdl in HSCs in a comprehensive and unbiased manner, we set out to identify HectD1 substrates by affinity purification of HectD1 interacting protein complex using mass spectrometry (MS). We transfected HA-tagged HectD1 or vector alone into HEK293T cells. HectdD1-interacting proteins were immunoprecipitated (IP) using anti-HA agarose beads, followed by HA peptide affinity elution. Glycine elution after HA elution detected very few bound proteins, and the non-specific proteins remained associated with the agarose beads upon boiling in SDS loading buffer, indicating the specificity and robustness of HA-affinity purification (Figure 3E). We thus subjected the HA eluates to MS analysis along with vector control. Triplicates of IP-MS were performed and results were evaluated using the CRAPome (Contaminant Repository for Affinity Purification) analysis (Mellacheruvu et al., 2013). We ranked and selected the potential interacting proteins with the cutoff of SAINT (Significance Analysis of INTeractome) score $\geq 0.5$ and fold change (FC) $\geq 3$ (Figure 3F). Gene ontology (GO) analysis revealed a high enrichment of ribosome/translationrelated proteins and proteasome proteins (Figure S5A and Supplementary Table S1), which is in agreement with the role of HectD1 as an E3 ubiquitin ligase and regulator of cell growth. Additionally, it suggested a potential role for HectD1 in ribosomes and protein synthesis.

Among the top hits, we focused on ZNF622 protein, given the critical role of its yeast homolog Reil in ribosome biogenesis (Greber et al., 2016; Meyer et al., 2010). The interaction between HectD1 and ZNF622 was first confirmed in Flag-ZNF622 reconstituted TF-1 cells by co-IP (Figure 3G). Next, we generated a series of deletion mutants of HectD1 and ZNF622, and transfected them into $293 \mathrm{~T}$ cells, followed by IP/WB analysis to map the responsible domain(s) for their interaction. Our data showed that Hectd1 interacted with ZNF622 regardless of its E3 activity as both HA-Hectd1 WT, and E3 dead mutant C2579G (Mut), but not vector control could pull down endogenous ZNF622. We also found that the Sad1/UNC domain of HectD1 is 
responsible for its interaction with ZNF622 (Figures S5B and S5C). Furthermore, deletion of either ZnF2 domain or the first linker region (LR1) located in the N-terminus of ZNF622 completely abolished its interaction with HectD1 (Figures S5D and S5E). Interestingly, cryo-EM analysis has revealed that that these two regions of Reil are located outside of the polypeptide tunnel (Greber et al., 2016; Kargas et al., 2019), rendering it accessible to HectD1 interaction.

Next, we asked if HectD1 regulates ZNF622 ubiquitination and protein stability. We found that Hectdl deficiency increased ZNF622 protein, but not mRNA levels in LSK cells (Figures 3H-3J). Consistently, ZNF622 protein but not mRNA level was increased in HECTD1-depleted TF-1 cells (Figures 3K, 3L and S5F). Importantly, the half-life of ZNF622 proteins was significantly prolonged in HECTD1-depleted TF-1 cells in the presence of cycloheximide (CHX) that blocks nascent protein synthesis (Figures $3 \mathrm{~K}$ and 3L). Next, we analyzed ZNF622 ubiquitination status impacted by HectD1 using Nickel-beads (Ni-NTA) pulldown of His-tagged Ub under denatured conditions to capture direct Ub-conjugation in ZNF622 proteins while reducing the detection of its associated proteins. Our data demonstrated that ZNF622 was robustly ubiquitinated by WT HectD1, but not the C2579G mutant. Moreover, consistent with the notion of HectD1 being an E3 ligase for lysine ${ }^{63}$-polyubiquitination (K63-Ub) (Sarkar and Zohn, 2012), K63R, but not K48R Ub

mutant, abolished ZNF622 ubiquitination (Figure 3M), further demonstrating that ZNF622 is a direct substrate of HectD1. Taken together, our data suggest that HectD1 interacts with, ubiquitinates and regulates the stability of ZNF622.

\section{Hectd1 insufficiency decreases HSPC proliferation and global translation rates upon stress}

The HectD1 IP-MS data and the interaction between HectD1-ZNF622 promoted us to investigate a potential role for HectD1 in protein synthesis and ribosome biology. To test this, we performed in vivo OP-puro (O-propargyl Puromycin) assay in primary adult Hectdl ${ }^{f l f}$ and Hectdl ${ }^{\text {flf:Vav }}$ mice 
to evaluate protein synthesis rate (Liu et al., 2012). We found that HSCs exhibited a lower protein synthesis rate than restricted progenitors and mature hematopoietic cells (Figures S5G and S5H), consistent with the previous report (Signer et al., 2014). However, Hectdl insufficiency did not affect protein synthesis rates in any of the hematopoietic cell subsets at the steady state (Figure $\mathrm{S} 5 \mathrm{H})$.

Our data showed that Hectdl deficiency does not affect phenotypic HSC number in the steady state (Figure S2G), but dramatically decreases functional HSCs in the transplantation assay or ex vivo culture, both of which conditions force HSCs to proliferate (Figures 1 and 2). Thus, we investigated if HectD1 is critical for HSC proliferation in vivo under stress conditions. We subjected the mice to two different types of stress. We first challenged Hectdl ${ }^{f l f}$ and Hectdl $I^{f l f ; V a v}$ mice with cytoablative drug 5-fluorouracil (5-FU), which depletes cycling hematopoietic cells and drives primitive $\mathrm{HSCs}$ to regenerate. At day 10 after 5-FU administration, Hectdl ${ }^{\text {flf:Vav }}$ mice exhibited a pronounced reduction in BM HSC and MPP numbers in comparison to the control mice (Figure 4A). Notably, both Hectdl deficient HSCs and MPPs showed reduced protein synthesis rate using the OP-Puro assay (Figures 4B and 4C), which correlated with their slower cycling status than the controls as determined by BrdU incorporation (Figure 4D). We did not observe elevated cell death under 5-FU stress (Figure S5I). To consolidate the conclusion of HectD1 function under stress conditions, we subjected mice to cyclophosphamide treatment followed by two daily doses of GCSF to induce HSPC proliferation (Morrison et al., 1997). In agreement, our data revealed lower HSC and MPP numbers (Figure 4E), slower cell cycle kinetics, as well as decreased protein synthesis rate in Hectdl ${ }^{\text {flf;Vav }}$ mice in comparison to the controls (Figures 4F-4H). 
Next, we examined OP-Puro incorporation in ex vivo cultured LSK cells, which were also undergoing proliferative stress. Hectdl-deficient cells displayed a decrease in global translation rate by $>20 \%$ when compared to control LSKs (Figures $4 \mathrm{I}$ and $4 \mathrm{~J}$ ). Consistently, Hectdl-deficient $\mathrm{BM}$ progenitors were hypersensitive to puromycin, an inhibitor of translation elongation (Figure 4K). Of note, this observed translation defect in both primary LSK cells and TF-1 cells was independent of eIF2 $\alpha$ or $4 \mathrm{E}-\mathrm{BP} 1$ phosphorylation, two key regulators of translation initiation (Figures S4B-S4E) (Holcik and Sonenberg, 2005). Taken together, we demonstrate that HectD1 plays a critical role in HSPC proliferation and protein synthesis under hematopoietic stress.

\section{Hectd1 deficiency disrupts ZNF622-mediated 60S ribosome maturation and 60S/40S subunit joining}

To dissect molecular mechanisms by which HectD1 regulates ribosome assembly and protein translation, we examined whether HectD1 affects ribosome composition by polysome profiling assay. Cell lysates of equal RNA content were fractionated in a sucrose gradient, followed by recording of the ultraviolet (UV) absorbance of each fraction. Hectd1-null LSK cells displayed an increased $60 \mathrm{~S}$ content but decreased $80 \mathrm{~S}$ monosome and polysome, while $40 \mathrm{~S}$ remained unchanged (Figure 5A). This phenotype was also observed in HECTD1-depleted TF-1 cells (Figure 5B). These data indicated a previously-unrecognized role for HectD1 in regulating ribosome assembly and proper global translation.

During ribosome biogenesis, subunits are exported to the cytoplasm as pre-60S and pre-40S complexes that must undergo final maturation involving sequential addition and release of a series of proteins to form mature 60S and 40S subunits (de la Cruz et al., 2015; Kargas et al., 2019). In yeast, ZNF622 homolog Rei1, stabilizes Arx1 (human PA2G4) association with pre-60S (Greber et al., 2012). Jjj1 (human DNAJC21) is required for the release of Rei1-Arx1 complex and the 
completion of polypeptide exit tunnel (PET) maturation (Lo et al., 2010; Meyer et al., 2010). Failure of Rei1/Arx1 dissociation from pre-60S particles precludes downstream assembly events, including the anti-association factor eIF6 release from pre-60S, which is a crucial step for $40 \mathrm{~S}$ joining to form a functional ribosome. Thus, we sought to test if the elevated ZNF622 level in HECTD1-depleted cells would affect 60S maturation. Fractions of shLuc or shHECTD1 TF-1 cells from the sucrose gradient were collected and examined by WB. The results showed that HECTD1 was predominantly located in the cytosol, with a small fraction detected in free ribosome subunits (Figure 5C). Notably, ZNF622 was predominantly associated with the 60S, and HECTD1 depletion resulted in a marked accumulation of ZNF622 in the 60S (Figures 5C and 5D). In contrast, the 40S protein Nob1 remained unchanged. Moreover, the ZNF622 binding partner PA2G4 and downstream eIF6 were markedly increased in the 60S, indicating an abnormal 60S ribosome formation (Figures 5C and 5D). Interestingly, the ribosome biogenesis factor for the maturation of the polypeptidyl transferase center (PTC), NMD3, was unchanged (Figures 5C and S6), indicating that the regulation of PET and PTC may be uncoupled. Besides the association and release of assembly factors, the cytoplasmic maturation of pre-60S subunit involves the concomitant incorporation of cytoplasmic ribosome proteins (de la Cruz et al., 2015; Kargas et al., 2019). Consistent with this notion, we found impaired incorporation of cytoplasmic RPL24 in the polysomes of HECTD1-depleted cells (Figures 5C and 5D), whereas other core RPL proteins that were pre-assembled in the nucleus, such as RPL11 and RPL23A, were unaffected (Figures 5C and S6). Of note, we observed a reduced pRPS6 level but not total RPS6 in HECTD1 deficient cells when compared to the controls (Figures 5C and S6). Together, these data suggest that increased ZNF622 proteins upon HECTD1 loss leads to a defective 60S maturation or activation of a translational quality control pathway arising from a block in the release of PA2G4 and eIF6, reduced joining of $60 \mathrm{~S}$ and $40 \mathrm{~S}$, thereby resulting in reduced $80 \mathrm{~S}$ and polysomes. 
To gain further insight into the role for HectD1 in the regulation of ZNF622 in 60S formation and $60 \mathrm{~S} / 40 \mathrm{~S}$ subunit joining, we assessed if depletion of ZNF622 could rescue the ribosome defects observed upon HECTD1 loss. We compared the polysome profiling and eIF6 retention in the 60S of shLuc control, HECTD1 single and HECTD1; ZNF622 double knockdown (DKD) TF-1 cells (Figure 5E). Our data showed that knockdown of ZNF622 in HECTD1-deficient cells restored ribosome composition as well as eIF6 release to normal levels (Figures 5F-5H). Furthermore, we performed ribosome dissociation/reassociation assay with these cell lines to directly interrogate 60S/40S subunit joining (Burwick et al., 2012). 80S monosomes and polysomes were first dissociated into 40S and 60S subunits under low $\mathrm{Mg}^{2+}$ conditions, and the total amount of $40 \mathrm{~S}$ and 60S was comparable among these three cell lines (Figures 5I and 5J). We subsequently added back $\mathrm{Mg}^{2+}$ allow 40S and 60S subunits to reassociate. HECTD1 depletion reduced ribosomal reassociation. Importantly, this disruption in ribosomal reassociation was reversed by ZNF622 knockdown (Figures 5I and 5J). Taken together, these results provide direct evidence that HectD1 plays an essential role in controlling 60S/40S subunit joining and translational control by regulating ZNF622.

\section{ZNF622 depletion restores protein translation and HSC transplantation activity induced by}

\section{Hectd1 loss}

We reasoned that if the compromised translation rate observed in Hectdl-deficient cells was owing to the elevated ZNF622 level and ZNF622-mediated ribosome defects, attenuation of ZNF622 expression would be able to rescue this phenotype. To test this hypothesis, we knocked down ZNF622 with shRNA in Luc- or HECTD1-depleted TF-1 cells (Figure 6A). Strikingly, ZNF622depletion fully restored protein synthesis rate to normal levels in HECTD1-depleted cells, while it did not significantly affect the translation rate in control cells as examined by the OP-Puro assay 
(Figure 6B). Of note, ZNF622 downregulation restored pRPS6 level in HECTD1-depleted cells that was independent of mTOR (Figures 6A and S6G).

We next asked if the aberrant accumulation of ZNF622 accounts for the perturbed HSC activity owing to Hectdl deficiency. To functionally test this, we depleted Znf622 in Hectdl-null HSCs and examined their reconstitution ability. We first generated two efficient shRNAs against mouse Znf622 (shZnf622\#1 and \#2) with \#1 shRNA being the most robust (Figure 6C). Subsequently, LSK cells were purified from Hectdl ${ }^{f f ; \text { Vav }}$ mice and infected with lentivirus expressing shLuc or two shRNAs to Znf622 with mCherry as a fluorescent marker. We injected 250k lentivirallyinfected LSKs into each recipient with 500k Sca1-depleted competitor BM cells to ensure high donor reconstitution and recipient survival (Figure 6D) (Jiang et al., 2012). Both Znf622 shRNAs increased the reconstitution of Hectdl-deficient HSCs in the peripheral blood (PB) of the recipient mice (Figure 6E). Importantly, mice transplanted with LSKs depleted of Znf622 had a significantly higher proportion of $\mathrm{mCherry}^{+}$cells in the BM HSCs and MPPs than those with shLuc (Figure 6F). Therefore, the restored donor chimerism observed in the PB was resulted from HSC restoration.

We next examined if the rescue effect of Znf622 downregulation was specific to Hectdl-null background. LSKs from both Hectdlfff and Hectdlff; Vav mice were sorted and infected with lentivirus expressing shLuc or shZnf622\#1 followed by BMT. While control HSC reconstitution in the PB was not significantly affected by Znf622 knockdown, Hectdl-deficient HSC reconstitution ability was significantly rescued (Figure 6G), and importantly, that was attributed to restored HSPC populations (Figure $6 \mathrm{H}$ ). Strikingly, mCherry ${ }^{+}$donor chimerism in the PB of secondary recipients further validated the HSC promoting effect of Znf622 knockdown as the reconstitution ability of Znf622-depleted Hectdl-null cells was significantly elevated in comparison to control cells (Figure 6I). To summarize, these data support a working model that 
ZNF622 is an important functional mediator of HectD1 function in regulating ribosome assembly and protein translation efficiency, as well as HSC regeneration.

\section{Discussion}

Tightly-regulated protein synthesis rate is critical for HSC maintenance and function, as only a $30 \%$ decrease (using $R p l 24^{B s t /+}$ mice, where ribosome protein Rpl24 is partially depleted) or increase (cKO of Pten or $4 E-B P 1 / 2$ mice) in protein synthesis is sufficient to impair HSC proliferation and self-renewal (Signer et al., 2014; Signer et al., 2016). Here we identified a critical role for ubiquitin-dependent regulation of ribosome assembly by HectD1 to meet the increased protein demands during $\mathrm{HSC}$ regeneration in vivo and ex vivo.

We demonstrate that HectD1 is required for HSC but not progenitor cell expansion in vivo, pointing to that balanced protein synthesis is essential for HSC function. Interestingly, HectD1 is dispensable for $\mathrm{HSC}$ development during homeostasis, but is critical for $\mathrm{HSC}$ regeneration under proliferative stress. Of note, HectD1 is found indispensable in all hematopoietic stress conditions we tested, such as in vivo transplant settings, ex vivo expansion under cytokines, as well as genotoxic stress 5-FU or cyclophosphamide/GCSF-induced HSPC proliferation. The extraordinary demands for HSPC growth and proliferation in these conditions require increased global protein production, thereby coordinated ribosome production. The data in this report suggest that HectD1 controls ribosome assembly and protein synthesis rate during HSC regeneration by regulating $60 \mathrm{~S}$ assembly factor ZNF622. Of note, we cannot exclude the possibility that HectD1 also regulates protein synthesis and cell cycle in some, and potentially many, hematopoietic progenitors after injury. 
Impaired ribosome function arising from genetic aberrations of RP proteins or assembly factors causes a group of human disorders known as "ribosomopathies", such as SDS. 90\% of SDS patients harbor mutations in the SBDS gene, which functions with the GTPase EFL1 to facilitate the removal of anti-association factor eIF6 from pre-60S ribosomal subunits to allow the assembly of 40S and 60S into functional monosomes (Finch et al., 2011; Menne et al., 2007; Warren, 2018; Weis et al., 2015). In addition, biallelic mutations in DNAJC21, a maturation factor for the PET, cause abnormal accumulation of PA2G4 and eIF6 in pre-60S ribosomal subunits and reduce 60S and 40S joining, eliciting an SDS-like phenotype (Dhanraj et al., 2017; Tummala et al., 2016). It is noteworthy that DNAJC21 assists the release of ZNF622-PA2G4 from pre-60S, allowing for the progression of downstream maturation steps. Thus, our data suggest that Hectdl deficiency recapitulates both the molecular ribosomal abnormalities and the phenotypic perturbations in HSCs, reminiscent of SDS. We found that depletion of ZNF622 in HECTD1-deficient cells rescues ribosome composition as well as eIF6 release to normal levels, thereby restoring 60S/40S subunit joining and protein translation. Notably, it also supports the idea that ZNF622 influences the affinity of eIF6 for the ribosome, thereby serving as a quality control step to ensure proper ribosome assembly. More importantly, we demonstrate that downregulation of Znf622 rescues HSC reconstitution capacity in Hectdl-null mice, implicating ZNF622 inhibition as a potential therapeutic strategy for the treatment of BMF disease with defective ribosomes.

It is important to point out that structure-function studies of ribosome biogenesis and assembly factors have been most examined in yeast and prokaryotes. Our work provides significant biochemical and functional insight into the critical biogenesis factors of human and mouse ribosomes. Structural analysis of the yeast homolog of ZNF622, Rei1, revealed that the Rei1 Cterminus is deeply inserted into the 60S PET, which is essential for the proofreading of PET maturation and subsequent Arx1 (yeast PA2G4) liberation and eIF6 eviction steps (Greber et al., 
2016). Our data in human hematopoietic cells demonstrate that accumulated ZNF622 in pre-60S blocks PA2G4 removal and efficient ribosome maturation, in accordance with the function of Rei1 in Arx1 release in yeast cells (Meyer et al., 2010). An alternative but not mutually-exclusive explanation points to a subunit joining and translation defect rather than a biogenesis defect upon HECTD1 loss, since the amount of total 40S and 60S was unaffected. In the absence of HECTD1, ZNF622 abnormally accumulates in the pre-60S and/or rebinds to mature $60 \mathrm{~S}$ to block $60 \mathrm{~S} / 40 \mathrm{~S}$ joining via ZNF622-associated eIF6. A role for ZNF622 and eIF6 in ribosomal stress and translational quality control has been suggested in a whole-genome CRISPRi screen in human chronic myeloid leukemia (CML) cell line. This study revealed that depletion of ZNF622 or eIF6 restores cell fitness and cell growth in the presence of PF8503, a translational inhibitor that binds to the PET and inhibits the translation of selective proteins and suppresses cell proliferation (Liaud et al., 2019). Hence, our work may uncover a ribosome quality control pathway that is critical for HSPCs during regenerative or proliferative stress. Under this circumstance, high demand for protein synthesis increases the need for ribosome quality control, where HECTD1 activity controls ZNF622 and eIF6-mediated ribosome assembly and protein translation efficiency.

In yeast, Rei1-Arx 1 departure from $60 \mathrm{~S}$ coincides with the exchange for the Rei1 family member Reh1 in the PET that persists in the later stages of cytoplasmic maturation process (Kargas et al., 2019; Ma et al., 2017). This finding is in striking contrast to those reported in yeast, in which dual knockout of Rei1 and Reh1 severely constrains yeast cell growth (Greber et al., 2016). Therefore, it is possible that ZNF622 exerts a distinct function from its yeast homologs. The N-terminus of Rei1 contacts RPL24 on the surface of the 60S ribosome (Greber et al., 2016) and directly interacts with eIF6 (Kargas et al., 2019). ZNF622 may regulate eIF6 release through its direct interaction with eIF6, or indirectly through RPL24. In agreement, we demonstrate that increased ZNF622 protein level coincides with an accumulation of eIF6 in the 60S subunit of HECTD1-depleted cells 
and depletion of ZNF622 reduces eIF6 association in the 60S. Of note, RPL24 is essential for the formation of $60 \mathrm{~S}-40 \mathrm{~S}$ inter-subunit bridges, one of which depends on the direct interaction between RPL24 and RPS6 (Kisly et al., 2019). Our data raise the possibility that RPS6 phosphorylation serves as a feedback regulation in the $40 \mathrm{~S}$ subunit when the $60 \mathrm{~S}$ large subunit encounters stress, as we observed a correlation between pRPS6 and 60S maturation and protein synthesis in this context. Nonetheless, the intricate regulation between different ribosome subunits and monosomes/polysomes mandates future investigations.

Taken together, we reveal a ubiquitin-dependent control of ribosome assembly and protein synthesis that is essential to HSC activity and regeneration, highlighting the importance of ribosome assembly factors in HSC function. Importantly, we demonstrate that Znf622 depletion restores $60 \mathrm{~S}$ ribosome maturation, ribosome assembly, protein synthesis and HSC activity in the context of Hectdl deficiency. This finding establishes an in vivo example of genetic suppression of HSC defects associated with dysfunctional ribosomes. Hence, it will likely enhance our understanding of the pathogenesis and therapeutic strategies for ribosomopathies.

\section{Limitations of the Study}

Our work demonstrates that the E3 ubiquitin ligase HectD1 plays an important role in HSC regeneration by ubiquitin-dependent regulation of ribosome assembly via ZNF622. While our study provides strong evidence for HectD1/ZNF622-mediated protein translation in HSPCs under stress conditions, it is unclear if they play a role in $60 \mathrm{~S}$ ribosome biogenesis or quality control of stressed ribosomes. We also found that the level of phospho-RPS6 was dramatically decreased in Hectdl-deficient cells and this correlates with reduced protein synthesis rate and HSPC function. However, we do not understand the underlying mechanism or its relevance to ZNF622. Another limitation is that we immunoblotted and quantified the distribution of ribosomal proteins and 
association factors in ribosomal fractions of a hematopoietic cell line TF-1 cells, but not in HSPCs due to their scarcity.

\section{Acknowledgements}

WT is supported by NIH grants R01DK127738 and R01HL095675, awards from Department of

Defense, St. Baldrick's Foundation, Alex's Lemonade Research Foundation, and Basser Center for BRCA Research. VRP is supported by an NIH grant R35 GM138035. AJW is supported by a Specialist Programme from Blood Cancer UK (12048, to AJW), the UK Medical Research Council (MR/T012412/1), the Kay Kendall Leukaemia Fund, a Wellcome Trust strategic award to the Cambridge Institute for Medical Research (100140), a core support grant from the Wellcome Trust and MRC to the Wellcome Trust-Medical Research Council Cambridge Stem Cell Institute, the Connor Wright Project, the Cambridge National Institute for Health Research Biomedical Research Centre and the European Cooperation in Science and Technology (COST) Action CA18233 "European Network for Innovative Diagnosis and treatment of Chronic Neutropenias, EuNet INNOCHRON". YC is supported by the Scientific and Technical Foundation of Shanxi Province (grant no. 2020JM-015). We thank Dr. Irene E. Zohn for kindly providing the HectD1 WT and C2579G constructs. We are grateful to Drs. John Woolford and Sebastian Klinge for valuable advice and helpful discussions of this work.

\section{Author contributions}

W. T. conceived the project and supervised the studies. W. T., K. L. designed the experiments and wrote the manuscript with input from all authors. K. L. performed all the animal experiments with assistance of C. G. and H. X., who did animal dissection and flow cytometry. R.D. performed initial biochemistry and colony assays. Y. C. helped perform transplantation assay. K. L., C. G. and J. R. contributed to immunoblotting experiments. K. L., V. R. P, C. A. and C. G. designed and 
performed polysome profiling assay. S.P. and A. J. W. contributed to ribosome assays and manuscript editing.

\section{Declaration of interests}

The authors declare no competing financial interests.

\section{Inclusion and diversity}

We worked to ensure sex balance in the selection of non-human subjects. The author list of this paper includes contributors from the location where the research was conducted who participated in the data collection, design, analysis, and/or interpretation of the work. 


\section{Main figure titles and legends}

Figure 1. Hectd1-deficient BMs display a defective reconstituting ability and reduced functional HSC frequency.

(A) Experimental scheme of serial BM transplantation assay.

(B) Representative flow plots of donor/competitor/host chimerism in the peripheral blood (PB)

of recipient mice after transplantation.

(C) Donor chimerisms in the PB of recipient mice were measured every 4 weeks and the results are graphed. Hectdl ${ }^{f f f}(\mathrm{n}=11)$ and Hectdl ${ }^{f l f ; \operatorname{Vav}}(\mathrm{n}=11)$.

(D) Lineage reconstitutions of donor-derived cells in primary recipients at 16 weeks posttransplantation are shown. Hectdl $I^{f / f}(\mathrm{n}=11)$ and Hectdl ${ }^{\text {flf; Vav }}(\mathrm{n}=11)$.

(E) Percentages of donor-derived HSPC subpopulation in the BM of primary transplanted mice at 16 weeks are shown. Hectdl ${ }^{f l f}(\mathrm{n}=6)$ and Hectdl ${ }^{f f f ; V a v}(\mathrm{n}=6)$.

(F) Donor percentages in the PB of secondary BMT recipients were analyzed every four weeks and the results are graphed. Hectdl ${ }^{f l f}(\mathrm{n}=10)$ and Hectdl ${ }^{\text {ff; } \text { Vav }}(\mathrm{n}=11)$.

(G) Experimental scheme of limiting dilution BMT to assess functional HSC frequency of Hectdl ${ }^{f l f}$ and Hectdl ${ }^{f f ; \text { Vav }}$ BMs. (H) The results are presented as number of positively engrafted mice versus total number of mice analyzed for the indicated doses. Positive engraftment was defined as $>1 \%$ donor-derived cells in the PB. CRU: competitive repopulating unit. 1SE: one standard deviation.

(I) Donor chimerisms in the PB of recipient mice transplanted with different doses of BM cells at 16 weeks are shown. 100k: Hectdl ${ }^{f / f}(\mathrm{n}=5)$ and Hectdl ${ }^{f f ;} ; \operatorname{Vav}(\mathrm{n}=6) ; 30 \mathrm{k}$ : Hectdl ${ }^{f / f}(\mathrm{n}=10)$ and $\operatorname{Hectdl}^{f f f ; \operatorname{Vav}}(\mathrm{n}=13) ; 10 \mathrm{k}$ : Hectd ${ }^{f f f}(\mathrm{n}=9)$ and Hectdl $I^{f f f \operatorname{Vav}}(\mathrm{n}=7)$. In all relevant panels, each symbol represents an individual mouse; bars indicate mean frequencies; error bars indicate SE. *: $\mathrm{p}<0.05 ; * *: \mathrm{p}<0.01 ; * * *: \mathrm{p}<0.001$; ns: not significant, as determined unpaired by two-tailed Student's $t$-test.

See also Figure S1, S2 and S3A-S3K.

Figure2. HectD1 is required for HSC self-renewal in vivo and maintenance ex vivo.

(A) Experimental scheme of HSC transplantation assay. LT-HSCs (LSK CD $150^{+} \mathrm{CD}^{-} 8^{-}$) from Hectd $l^{f f f}$ and Hectd ${ }^{f f ; \text { Vav }}$ mice were purified by flow cytometric sorting and 100 HSCs were either injected with 500K Sca1-depleted competitor BMs into lethally irradiated recipient mice (Day0BMT) or the resultant culture after 12 days was injected with $300 \mathrm{~K}$ BMs into recipient mice (Day12-BMT).

(B) Donor chimerisms in the PB of recipient mice transplanted with fresh HSCs (day0-BMT) were measured every 4 weeks and the results are shown in the graph. Hectdl $I^{f / f}(\mathrm{n}=8)$ and Hectdl $I^{f l f ; V a v}$ $(n=8)$.

(C) Percentages of donor-derived HSPC subpopulations in the BM of day0-BMT recipient mice 16 weeks post-transplant are shown. Hectdl ${ }^{\text {flf }}(\mathrm{n}=6)$ and Hectdl $1^{f f f: V a v}(\mathrm{n}=5)$.

(D) Donor chimerisms of day12 cultured HSC transplants (Day12-BMT) in the PB of recipient mice were measured every 4 weeks and the results are shown in the graph. Hectdl $I^{f / f}(\mathrm{n}=7)$ and Hectdl $1^{f f f: \operatorname{Vav}}(\mathrm{n}=5)$.

(E) Percentages of donor-derived HSPC subpopulations in the BM of day12-BMT recipient mice 16 weeks post-transplant are shown. Hectdl ${ }^{f l f}(\mathrm{n}=6)$ and Hectdl ${ }^{f l f}$;Vav $(\mathrm{n}=4)$.

(F) Representative images of ex vivo cultured HSCs at day 8.

(G-I) Cell numbers of ex vivo cultured HSCs at different time points in different combinations of cytokines are shown. $\mathrm{n}=3$ in each group.

(J) Experimental scheme of HSC versus MPP/HPC transplantation assay. HSCs (LSK $\mathrm{CD} 150^{+} \mathrm{CD}_{4} 8^{-}$) or MPP/HPCs (LSK CD150-CD48 ${ }^{+}$) were sorted from Hectdl ${ }^{f l f}$ and Hectdl ${ }^{\text {flf; Vav }}$ mice. $500 \mathrm{HSCs}$ or $5000 \mathrm{MPP} / \mathrm{HPCs}$ were transplanted into each sub-lethally irradiated recipient 
mice.

(K-L) Donor chimerisms in the $\mathrm{PB}$ of recipient mice were measured by flow cytometry every week post-BMT. Donor chimerisms of HSC (K) and MPP/HPC (L) transplants are shown. Hectdl flf $(\mathrm{n}=7-9)$ and Hectdl ${ }^{\text {flf; } \operatorname{Vav}}(\mathrm{n}=6-7)$.

Data in (G-I) are represented by mean \pm SD. In all relevant panels, each symbol represents an individual mouse; bars indicate mean frequencies; error bars indicate $\mathrm{SE}$. $*: \mathrm{p}<0.05 ; * *: \mathrm{p}<0.01$; $* * *: \mathrm{p}<0.001$, as determined by unpaired two-tailed Student's $t$-test.

See also Figure S3L-S3M.

Figure 3. HectD1 interacts with, ubiquitinates, and degrades ZNF622.

(A) Freshly purified LSKs from Hectdl flf and Hectdl fff; Vav mice were used to examine various signaling molecules by WB using the indicated antibodies.

(B) TF-1/hMPL cells stably depleted of HECTD1 using two different shRNAs were generated along with shRNA to Luciferase (Luc). Cell lysates were subjected to WB analysis using indicated antibodies.

(C, D) TF-1/hMPL shLuc or shHECTD1 cells were cultured in triplicates in different concentrations of GM-CSF (D) or TPO (D). Cell growth after 3 days' culture were determined by MTT absorbance.

(E) Silver staining gel image of a representative large-scale protein purification result to evaluate the efficiency and specificity of affinity purification of HA-HectD1 interacting proteins. * indicates the HA-HectD1 bait. IgG-H: indicates the Immunoglobin heavy chain.

(F) CRAPome analysis of Hectd1-intearacting proteins from three independent IP-MS results revealed the SAINT probability over fold changes. ZNF622 was identified as an Hectd1 interactor and highlighted in red.

(G) co-IP/WB analysis confirmed the interaction between Flag-ZNF622 and endogenous HectD1 in Flag-ZNF622 reconstituted TF-1 cells.

(H) ZNF622 protein levels were increased in Hectd1 ${ }^{\text {ff;:Vav }}$ LSKs compared to that of Hectd1 ${ }^{\text {flf }}$ LSKs.

(I) Quantification of ZNF622 protein levels from three independent experiments as in (H) is plotted.

(J) ZNF622 mRNA levels were not affected in Hectdl-deficient LSKs as shown by qRT-PCR analysis. $\mathrm{n}=3$ in each group.

(K) TF-1 cells stably depleted of HECTD1 using two different shRNAs were treated with cycloheximide (CHX) for indicated times. ZNF622 half-lives were determined by WB. Representative blots of 3 independent experiments are shown. S.E., short exposure; L.E., long exposure.

(L) Relative ZNF622 levels normalized to Luc time 0 (left panel) and that normalized to respective time 0 (right panel) as shown in (J).

(M) $293 \mathrm{~T}$ cells were transfected with HA-HectD1 or E3-dead mutant HectD1, along with FlagZNF622 and His-Ub or Ub mutant constructs as indicated. Cells were subjected to lysis in denatured condition followed by $\mathrm{Ni}^{2+}$ beads-pulldown. Ubiquitinated proteins were detected by WB using indicated antibodies.

In all relevant panels, data are represented by mean \pm SD. $p$-values are determined by unpaired two-tailed Students' t-test. *: $\mathrm{p}<0.05 ; * *: \mathrm{p}<0.01 ; * * *: \mathrm{p}<0.001$

See also Figure S4.

Figure 4. Hectdl deficiency reduces HSC frequency and protein translational rate upon proliferative stress

(A-D) Hectd $1^{f / f}$ and Hectdl ${ }^{\text {ff; } V a v}$ mice were injected with $150 \mathrm{mg} / \mathrm{kg}$ 5-FU, and euthanized at 10 days later for subsequent analysis. 
(A) HSC and MPP numbers in the BM of 5-FU challenged Hectdl ${ }^{f l f}(\mathrm{n}=8)$ and Hectdl ${ }^{\text {flf:Vav }}$ (n=8) mice are shown.

(B) Representative histogram plot of protein synthesis rate in BM HSCs of 5-FU challenged mice as determined by in vivo OP-Puro assay.

(C) Quantification of protein synthesis rate in HSCs and MPPs of 5-FU challenged Hectdl ${ }^{\mathrm{flf}}(\mathrm{n}=6)$ and Hectdl ${ }^{\text {flf:Vav }}(\mathrm{n}=5)$ mice as shown in (B).

(D) Percentages of BM HSCs and MPPs in the S phase of the cell cycle as determined by in vivo BrdU assay. Hectdl ${ }^{f / f}(\mathrm{n}=3)$ and Hectdl ${ }^{\text {flf; } \operatorname{Vav}}(\mathrm{n}=3)$.

(E-H) Hectdl ${ }^{f / f}$ and Hectdl ${ }^{\text {ff;:Vav }}$ mice were injected with cyclophosphamide (Cy) followed by two consecutive daily injections of G-CSF. Mice were euthanized one day after the last injection for subsequent analysis.

(E) HSC and MPP numbers in the BM of Cy+2GCSF challenged Hectd1 ${ }^{\text {flf }}(\mathrm{n}=7)$ and Hectd $1^{\text {flf; Vav }}$ $(n=7)$ mice. Data are pooled from 4 independent experiments and unique symbols indicate mice from different experiments.

(F) Representative histogram plot of protein synthesis rate in BM HSCs of Hectdl ${ }^{\text {flf }}$ and Hectdl flf; Vav mice as determined by in vivo OP-Puro assay.

(G) Quantification of protein synthesis rate in HSCs and MPPs of Hectdl ${ }^{f / f}(\mathrm{n}=4)$ and Hectdl flf; Vav $(\mathrm{n}=4)$ mice as shown in $(\mathrm{F})$.

(H) Percentages of BM HSCs and MPPs in the S phase of the cell cycle as determined by in vivo BrdU assay. Hectdl ${ }^{f l f}(\mathrm{n}=3)$ and Hectdl ${ }^{\text {flf; Vav }}(\mathrm{n}=4)$.

(I) Protein synthesis rates of 2-day cultured LSKs from Hectdl ${ }^{f / f}$ and Hectdl flf;Vav mice were determined by OP-puro incorporation of newly synthesized protein after $1 \mathrm{hr}$ labelling. Representative histogram plot is shown.

(J) Quantification of relative protein synthesis rates of 2-day cultured LSKs from three independent experiments using OP-Puro assays as shown in (I).

(K) Relative CFU-GM progenitors from_Hectdl ${ }^{f l f}(\mathrm{n}=3)$ and Hectdl flf;Vav $(\mathrm{n}=3)$ BMs in the presence of various concentrations of the translation elongation inhibitor puromycin is shown.

Data in (A, C, D, E, G and H) are represented by mean \pm SE. Data in (J, and K) are represented by mean \pm SD. $p$-value in $(E)$ is determined by paired two-tailed Students' t-test; $p$-values in other panels are determined by unpaired two-tailed Students' t-test. *: $p<0.05 ; * *: p<0.01 ; * * *: p<0.001$; ns, not significant.

See also Figure S5.

Figure 5. Hectd1 deficiency results in an accumulation of ZNF622 and eIF6 in the 60S and a reduction in ribosomal subunit joining, which is restored by ZNF622 depletion.

(A) Polysome profiling analysis of 2 day-cultured LSKs from Hectdl ${ }^{f l f}$ and Hectdl ${ }^{\text {fl; } \text { Vav }}$ mice.

(B) Quantifications of 60S:40S ratio (left panel) and 60S:80S ratio (right panel) from polysome profiling assay of TF-1 cells expressing shLuc or shHECTD1. Three independent experiments were performed.

(C) Fractions from sucrose gradients (7\%-45\%) of TF-1 cell lysates stably expressing shLuc or shHECTD1 were collected and subjected to WB analysis. Representative result of three independent experiments is shown. Fractions 1-3 are cytoplasmic soluble proteins. 40S, 60S, $80 \mathrm{~S}$ monosome and polysome fractions are indicated by colored lines, arrows, and fonts. Whole cell lysate (WCL). AF: assembly factor; RPL: ribosome protein large unit; RPS: ribosome protein small unit. WCL and sucrose fractions (shLuc and shHECTD1) were resolved in three SDS-PAGE gels in parallel. Sucrose fraction immunoblots were processed and developed in parallel, and images presented side-by-side. 
(D) Quantification of relative protein distribution in different polysome fractions as shown in (C). Relative protein levels in each fraction was normalized to the peak fraction of the indicated protein from the shLuc cells and plotted. $n=3-4$.

(E-J) Knockdown of ZNF622 in HECTD1-deficient cells rescues ribosome composition, eIF6 release, as well as $60 \mathrm{~S} / 40 \mathrm{~S}$ joining.

(E) WB examination of knockdown efficiency in shLuc, HECTD1 single and HECTD1;ZNF622 double knockdown (DKD) cells.

(F) Representative polysome profiles of TF-1 shLuc, HECTD1 and DKD cells.

(G) Quantifications of 60S:40S ratio (left panel) and 60S:80S ratio (right panel) of polysome profiles as shown in $(\mathrm{F}) . \mathrm{n}=3$.

(H) Representative result of WB analysis with protein fractions from sucrose gradients $(7 \%-45 \%)$ of TF-1 shLuc, HECTD1 and DKD cells (top panel). Quantification of eIF6 distribution in polysome fractions (bottom panel). $\mathrm{N}=3$.

(I) Ribosome dissociation/reassociation assay. Indicated TF-1 cell lines were lysed in $0.25 \mathrm{mM}$ low $\mathrm{Mg}^{2+}$ buffer to dissociate ribosomal subunits (Top graph). $\mathrm{MgCl}_{2}$ was subsequently added to a final concentration of $10 \mathrm{mM}$ for ribosomal subunit reassociation (Bottom graph). Resultant cell lysates were loaded on a $7-45 \%$ sucrose gradient profiled. Representative graphs from three independent experiments are shown.

(J) Quantification of 60S:40S ratios in the dissociated profiles (Top panel) and 80S:40S ratios in the reassociated profiles (Bottom panel). $\mathrm{N}=3$. Note that the black line (shLuc) and the blue line (DKD) in (F, H and I) superimpose.

All data are represented by mean \pm SD. p-values are determined by unpaired two-tailed Students' t-test. $*: \mathrm{p}<0.05 ; * *: \mathrm{p}<0.01 ; * * *: \mathrm{p}<0.001 ; \mathrm{ns}$, not significant.

See also Figure S6A-S6F.

Figure 6. Knockdown of Znf622 in Hectd1-deficient cells restores protein synthesis rate and HSC reconstitution ability.

(A) TF-1 cells stably expressing control shLuc, single or double knockdown of HECTD1 and ZNF622 were generated by lentiviral infection and sorting. WB analysis with indicated antibodies is shown.

(B) Global protein synthesis rates of various TF-1 cells as in (A) were measured using OP-Puro assay.

(C) Knockdown efficiency of 3 different shRNAs to mouse Znf622 in BaF3 cells is shown. shRNA \#1 and \#2 are chosen for subsequent BMT.

(D) Schematic illustration of HSC lentiviral transduction/BMT strategy.

(E) mCherry $^{+}$donor fractions in the PB were analyzed every 4 weeks post-BMT. Quantifications

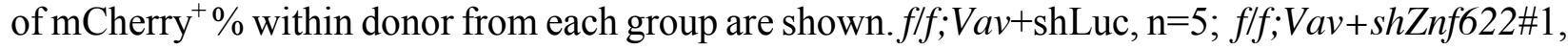

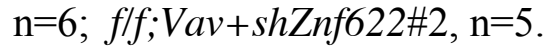

(F) Quantifications of mCherry $^{+}$donor\% in the HSC and MPP fractions 16-weeks post BMT are

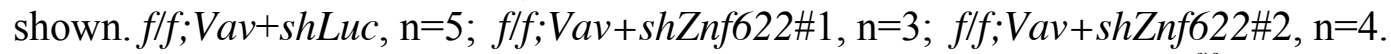

(G) In a separate experiment, LSK cells were purified from Hectdl ${ }^{f / f}$ and Hectdl ${ }^{\text {flf;Vav }}$ mice, infected with lentivirus expressing shLuc or shZnf622\#1, and subsequently transplanted. Quantifications of $\mathrm{mCherry}^{+}$donor\% in the PB from each group are shown. $f / f+s h L u c, \mathrm{n}=8$;

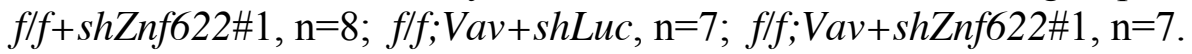

(H) Quantifications of $\mathrm{mCherry}^{+}$donor percentages in the HSC and MPP fractions at the end of

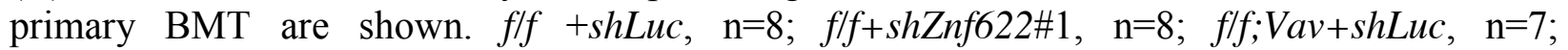

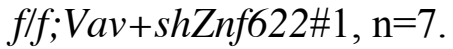

(I) Two million BM cells from primary transplanted mice were harvested and transplanted into each secondary recipient. Quantifications of mCherry $\%$ within donor from each group in the 
secondary transplants are shown. $f / f+\operatorname{sh} L u c, \mathrm{n}=16 ; f / f+\operatorname{shZnf622} \# 1, \mathrm{n}=13 ; f / f ; \operatorname{Vav}+\operatorname{sh} L u c, \mathrm{n}=14$;

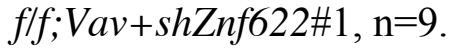

In all relevant experiments, each symbol represents an individual mouse; horizontal lines indicate mean frequencies; error bars indicate SE. *: $\mathrm{p}<0.05$; **: $\mathrm{p}<0.01$; ***: $\mathrm{p}<0.001$; ns, not significant, as determined by unpaired two-tailed Student's $t$-test.

See also Figure S6G.

\section{Supplemental Excel Table titles and legends}

Table S1. Fold enrichment changes and SAINT score for HectD1 interacting proteins by IP/MS. Related to Figure 3.

Table S2. Genotyping and qPCR primers used. Related to STAR Methods. 


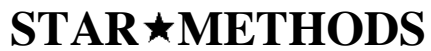

\section{KEY RESOURCES TABLE}

\begin{tabular}{|c|c|c|}
\hline REAGENT or RESOURCE & SOURCE & IDENTIFIER \\
\hline \multicolumn{3}{|l|}{ Antibodies } \\
\hline Rabbit polyclonal anti-HectD1 & Bethyl lab & Cat\# A302-908A, RRID:AB_10664800 \\
\hline Rabbit polyclonal anti-ZNF622 & Bethyl lab & Cat\# A304-075A, RRID:AB_2621324 \\
\hline Rabbit polyclonal anti-NOB1 & Bethyl lab & Cat\# A304-680A, RRID:AB_2620875 \\
\hline Rabbit polyclonal anti-RPS3 & Bethyllab & Cat\# A303-840A, RRID:AB_2620191 \\
\hline Rabbit polyclonal anti-RPL23A & Proteintech & Cat\# 16386-1-AP, RRID:AB_2269755 \\
\hline Rabbit polyclonal anti-RPL24 & Proteintech & Cat\# 17082-1-AP, RRID:AB_2181728 \\
\hline Rabbit polyclonal anti-eIF6 & Proteintech & Cat\# 10291-1-AP, RRID:AB_2096515 \\
\hline Rabbit polyclonal anti-NMD3 & Proteintech & Cat\# 16060-1-AP, RRID:AB_2282830 \\
\hline Rabbit polyclonal anti-DNAJC21 & Proteintech & Cat\# 23411-1-AP, RRID:AB_2879274 \\
\hline Mouse monoclonal anti-PA2G4 & Proteintech & Cat\# 66055-1-Ig, RRID:AB_11042597 \\
\hline Rabbit polyclonal anti-RPL11 & Abcam & Cat\# ab79352, RRID:AB_2042832 \\
\hline Rabbit monoclonal anti-RPL11 & Abcam & Cat\# ab32157, RRID:AB_732117 \\
\hline Mouse monoclonal anti- GSK $3 \alpha / \beta$ & Millipore & Cat\# 368662-200UG, RRID:AB_2043310 \\
\hline Mouse monoclonal anti-HSP90 & StressMarq & Cat\# SMC-107, RRID:AB_854214 \\
\hline Mouse monoclonal anti- $\beta$-Catenin & BD Biosciences & Cat\# 610153, RRID:AB_397554 \\
\hline Goat monoclonal anti-Actin & Santa Cruz & Cat\# sc-1616, RRID:AB 630836 \\
\hline Rabbit polyclonal anti-STAT5 & Santa Cruz & Cat\# sc-835, RRID:AB_632446 \\
\hline Rabbit monoclonal anti-pY1007/1008-JAK2 & Cell Signaling & Cat\# 3776, RRID:AB_2617123 \\
\hline Rabbit monoclonal anti-JAK2 & Cell Signaling & Cat\# 3230, RRID:AB_2128522 \\
\hline Rabbit polyclonal pY694-STAT5 & Cell Signaling & Cat\# 9351, RRID:AB_2315225 \\
\hline Rabbit polyclonal anti-pS473-AKT & Cell Signaling & Cat\# 9271, RRID:AB_32982 \\
\hline Rabbit polyclonal anti-AKT & Cell Signaling & Cat\# 9272, RRID:AB_329827 \\
\hline Mouse monoclonal anti-pT202/204-ERK1/2 & Cell Signaling & Cat\# 9106, RRID:AB_331768 \\
\hline Rabbit polyclonal anti-ERK1/2 & Cell Signaling & Cat\# 9102, RRID:AB_330744 \\
\hline Rabbit monoclonal anti-pS2448-mTOR & Cell Signaling & Cat\# 5536, RRID:AB_10691552 \\
\hline Mouse monoclonal anti-mTOR & Cell Signaling & Cat\# 4517, RRID:AB_1904056 \\
\hline Mouse monoclonal pT389-S6K1 & Cell Signaling & Cat\# 9206, RRID:AB_2285392 \\
\hline Rabbit polyclonal anti-S6K1 & Cell Signaling & Cat\# 9202, RRID:AB_331676 \\
\hline Rabbit monoclonal anti-pS235/236-RPS6 & Cell Signaling & Cat\# 4858, RRID:AB_916156 \\
\hline Rabbit polyclonal anti-pS240/244-RPS6 & Cell Signaling & Cat\# 2215, RRID:AB_331682 \\
\hline Rabbit monoclonal anti-RPS6 & Cell Signaling & Cat\# 2217, RRID:AB_331355 \\
\hline Rabbit monoclonal anti-pT37/46-4E-BP1 & Cell Signaling & Cat\# 2855, RRID:AB_560835 \\
\hline Rabbit monoclonal anti-4E-BP1 & Cell Signaling & Cat\# 9644, RRID:AB_2097841 \\
\hline Rabbit polyclonal anti-pS21/9-GSK3 $\alpha / \beta$ & Cell Signaling & Cat\# 9331, RRID:AB_329830 \\
\hline Rabbit polyclonal anti-eIF $2 \alpha$ & Cell Signaling & Cat\# 9722, RRID:AB_2230924 \\
\hline Rabbit monoclonal anti- $\beta$-Tubulin & Cell Signaling & Cat\# 2128, RRID:AB_823664 \\
\hline Mouse monoclonal anti-GAPDH & Cell Signaling & Cat\# 97166, RRID:AB_2756824 \\
\hline Rabbit monoclonal anti-HA & Cell Signaling & Cat\# 3724, RRID:AB_1549585 \\
\hline Mouse monoclonal anti-FLAG M2-HRP & Sigma-Aldrich & Cat\# A8592, RRID:AB_439702 \\
\hline Digital anti-mouse HRP & Kindle Biosciences & Cat\# R1005, RRID:AB_2800463 \\
\hline Digital anti-Rabbit HRP & Kindle Biosciences & Cat\# R1006, RRID:AB_2800464 \\
\hline CD45.1-FITC & BD Biosciences & Cat\# 553775, RRID:AB_395043 \\
\hline CD45.1-PE-Cy7 & Thermo Fisher & Cat\# 25-0453-82, RRID:AB_469629 \\
\hline CD45.1-eF450 & Thermo Fisher & Cat\# 48-0453-82, RRID:AB_1272189 \\
\hline CD45.2-APC-Cy7 & Thermo Fisher & Cat\# 47-0454-82, RRID:AB_1272175 \\
\hline CD45.2-Buv395 & BD Biosciences & Cat\# 564616, RRID:AB_2738867 \\
\hline Gr1-PE & Thermo Fisher & Cat\# 12-5931-83, RRID:AB_466046 \\
\hline Mac1-APC & Thermo Fisher & Cat\# 17-0112-83, RRID:AB_469344 \\
\hline B220-PE & BD Biosciences & Cat\# 553090, RRID:AB_394620 \\
\hline B220-APC & Thermo Fisher & Cat\# 17-0452-83, RRID:AB_469396 \\
\hline
\end{tabular}




\begin{tabular}{|c|c|c|}
\hline CD4-PE & BD Biosciences & Cat\# 553049, RRID:AB_394585 \\
\hline CD8a-PE & BioLegend & Cat\# 100707, RRID:AB_312746 \\
\hline CD3e-FITC & Thermo Fisher & Cat\# 11-0031-82, RRID:AB 464882 \\
\hline CD3e-PE & Thermo Fisher & Cat\# 12-0031-85, RRID:AB_465498 \\
\hline Ter119-biotin labeled & Thermo Fisher & Cat\# 13-5921-85, RRID:AB_466798 \\
\hline Gr1-biotin labeled & Thermo Fisher & Cat\# 13-5931-86, RRID:AB_466802 \\
\hline Mac1-biotin labeled & Thermo Fisher & 13-0112-86, RRID:AB_466361 \\
\hline B220-biotin labeled & Thermo Fisher & Cat\# 13-0452-86, RRID:AB_466451 \\
\hline CD19-biotin labeled & Thermo Fisher & Cat\# 13-0193-86, RRID:AB_657655 \\
\hline CD4-biotin labeled & Thermo Fisher & Cat\# 13-0041-86, RRID:AB_466327 \\
\hline CD5-biotin labeled & Thermo Fisher & Cat\# 13-0051-85, RRID:AB_466340 \\
\hline CD8a-biotin labeled & Thermo Fisher & Cat\# 13-0081-86, RRID:AB_466348 \\
\hline Streptavidin-PE-TexasRed & Thermo Fisher & Cat\# SA1017, RRID:N/A \\
\hline Streptavidin-APC-Cy7 & Thermo Fisher & Cat\# 47-4317-82, RRID:AB_10366688 \\
\hline c-Kit-PE & BD Biosciences & Cat\# 553355, RRID:AB_394806 \\
\hline c-Kit-APC & Thermo Fisher & Cat\# 17-1171-83, RRID:AB 469431 \\
\hline c-Kit-APC-Cy7 & Thermo Fisher & Cat\# 47-1171-82, RRID:AB_1272177 \\
\hline ScaI-PE & BD Biosciences & Cat\# 553336, RRID:AB_394792 \\
\hline ScaI-PerCp-Cy5.5 & Thermo Fisher & Cat\# 45-5981-82, RRID:AB_914372 \\
\hline CD150-PE-Cy7 & BioLegend & Cat\# 115914, RRID:AB_439797 \\
\hline CD48-FITC & BioLegend & Cat\# 103403, RRID:AB_313018 \\
\hline CD48-APC-Cy7 & BioLegend & Cat\# 103432, RRID:AB_2561463 \\
\hline CD48-AF700 & BioLegend & Cat\# 103426, RRID:AB_10612755 \\
\hline CD48-APC & Thermo Fisher & Cat\# 17-0481-82, RRID:AB 469408 \\
\hline Flk2-PE & BD Biosciences & Cat\# 553842, RRID:AB 395079 \\
\hline CD34-APC & Thermo Fisher & Cat\# 50-0341-82, RRID:AB_10596826 \\
\hline CD16/32-PE & BD Biosciences & Cat\# 553145, RRID:AB_394660 \\
\hline \multicolumn{3}{|l|}{ Bacterial and Virus Strains } \\
\hline pCL20.MSCV.mir30.PGK.mCherry & Holmfeldt et al., 2016 & $\mathrm{~N} / \mathrm{A}$ \\
\hline pCL20.MSCV.mir30.PGK.GFP & $\begin{array}{l}\text { This paper (Replace mCherry } \\
\text { with GFP) }\end{array}$ & N/A \\
\hline \multicolumn{3}{|l|}{ Chemicals, Peptides, and Recombinant Proteins } \\
\hline 5-Fluorouracil (5-FU) & Sigma-Aldrich & Cat\# F6627 \\
\hline Cyclophosphamide & Baxer & Cat\# NDC 10019-955-01 \\
\hline O-Propargyl-Puromycin (OP-Puro) & MedChemExpress & Cat\# HY-15680/CS-6850 \\
\hline BrdU & BD Pharmingen & Cat\# 5133284 \\
\hline Puromycin dihyrdochloride & Sigma-Aldrich & Cat\# P7255 \\
\hline Insulin Solution Human & Sigma-Aldrich & Cat\# I9278 \\
\hline $10 \%$ BSA in IMDM & StemCell Technologies & Cat\# 09300 \\
\hline L-Glutamine & Gibco & Cat\# 25030-081 \\
\hline Holo-Transferrin human & Sigma-Aldrich & Cat\# T0665 \\
\hline$\beta$-Mercaptoethanol & Sigma-Aldrich & Cat\# M3148 \\
\hline Propidium iodide $(\mathrm{PI})$ & Sigma-Aldrich & Cat\# P4170 \\
\hline DAPI & Sigma-Aldrich & Cat\# D9542 \\
\hline $3 \%$ Acetic Acid with Methylene Blue & StemCell Technologies & Cat\# 07060 \\
\hline Retronectin & Takara & Cat\# T100B \\
\hline Cycloheximide & Sigma-Aldrich & Cat\# C7698 \\
\hline Imidazole & Acros Organics & Cat\# 288-32-4 \\
\hline N-Ethylmaleimide (NEM) & Sigma-Aldrich & Cat\# E1271 \\
\hline PR619 & LifeSensors & Cat\# SI9619 \\
\hline 1,10-phenanthroline & Mallinckrodt Chemicals & Cat\# 2631-55 \\
\hline Sodium Fluoride & Sigma-Aldrich & Cat\# S7920 \\
\hline Sodium orthovanadate & Sigma-Aldrich & Cat\# 450243 \\
\hline PMSF & Sigma-Aldrich & Cat\# P7626 \\
\hline Protease Inhibitor Cocktail Tablets; EDTA-free & Roche & Cat\# 11836170001 \\
\hline Protease Inhibitor Cocktail Tablets & Roche & Cat\# 11697498001 \\
\hline Bovine Serum Albumin (BSA) & Fisher Bioreagents & Cat\# BP1600 \\
\hline
\end{tabular}




\begin{tabular}{|c|c|c|}
\hline Nonfat milk & Santa Cruz & Cat\# sc2325 \\
\hline StemSpan SFEM & StemCell Technologies & Cat\# 09600 \\
\hline Fetal Bovine Serum & SAFC Biosciences & Cat\# 12103C \\
\hline Bovine Calf Serum & HyClone & Cat\# SH30072.03 \\
\hline HA peptide & Thermo Fisher & Cat\# 26184 \\
\hline Anti-HA Affinity Gel & Sigma-Aldrich & Cat\# E6779 \\
\hline Anti-FLAG M2 Affinity Gel & Sigma-Aldrich & Cat\# F2426 \\
\hline Protein A Sepharose CL-4B & GE Health & Cat\# 17-0780-01 \\
\hline Protein G Sepharose 4 Fast Flow & GE Health & Cat\# 17-0618-01 \\
\hline HisPur Ni-NTA Resin & Thermo Fisher & Cat\# 88221 \\
\hline Murine SCF & Peprotech & Cat\# 250-03 \\
\hline Murine Tpo & Peprotech & Cat\# 315-14 \\
\hline Murine IL3 & Peprotech & Cat\# 213-13 \\
\hline Murine FLT3L & Peprotech & Cat\# 250-31L \\
\hline Murine IL6 & Peprotech & Cat\# 216-16 \\
\hline rh EPO & Espogen & Cat\# NDC 55513-144-10 \\
\hline rG-CSF (Neupogen) & Amgen & Cat\# NDC 55513-209-01 \\
\hline Human GM-CSF & Peprotech & Cat\# 300-03 \\
\hline Human TPO & Peprotech & Cat\# 300-18 \\
\hline \multicolumn{3}{|l|}{ Critical Commercial Assays } \\
\hline Lineage Cell Depletion Kit mouse & Miltenyi Biotec & Cat\# 130-090-858 \\
\hline Anti-Sca-1 Microbead Kit (FITC) & Miltenyi Biotec & Cat\# 130-092-529 \\
\hline Click-iT Plus OP-Puro Protein Synthesis Kit & Thermo Fisher & Cat\# C10458 \\
\hline FITC Annexin V Apoptosis Detection Kit & BD Pharmingen & Cat\# 556547 \\
\hline BrdU Flow Kits & BD Pharmingen & Cat\# 557891 \\
\hline BD Cytofix/Cytoperm & BD Biosciences & Cat\# 554714 \\
\hline SYBR Green Master Mix & Applied Biosystems & Cat\# 43-091-55 \\
\hline RNeasy Plus Mini Kit & QIAGEN & Cat\# 74136 \\
\hline qScript cDNA Supermix & Quanta Biosciences & Cat\# 98047 \\
\hline \multicolumn{3}{|l|}{ Experimental Models: Cell Lines } \\
\hline TF-1 & ATCC & Cat\# CRL-2003, RRID:CVCL_0559 \\
\hline HEK293T & ATCC & Cat\# CRL-3216, RRID:CVCL_0063 \\
\hline WEHI-3B & Dr. Harvey Lodish lab & RRID:CVCL_2239 \\
\hline BaF3 & DSMZ & Cat\# ACC-300, RRID:CVCL_0161 \\
\hline \multicolumn{3}{|l|}{ Experimental Models: Mouse Strains } \\
\hline C57B6/NGpt-Hectdl $I^{\mathrm{tmlb}} / \mathrm{Gpt}$ & EuComm and MARC, China & Cat\# 5757216, RRID:MGI:5757216 \\
\hline FLP B6;SJL-Tg(ACTFLPe)9205Dym/J & Jackson laboratories & Cat\# JAX:003800, RRID:IMSR_JAX:003800 \\
\hline Vav-Cre mice & $\begin{array}{l}\text { Stadtfeld and Graf, 2005; Chen } \\
\text { et al., } 2009\end{array}$ & N/A \\
\hline C57BL/6J (CD45.2) & Jackson laboratories & Cat\# 000664 \\
\hline SJL (CD45.1) & Jackson laboratories & Cat\# 000686 \\
\hline \multicolumn{3}{|l|}{ Oligonucleotides } \\
\hline sh human HECTDI\#1: tatgaaacaagattgtagtcaa & This paper & $\mathrm{N} / \mathrm{A}$ \\
\hline sh human HECTD1\#2: taccactggttgttcaactcta & This paper & $\mathrm{N} / \mathrm{A}$ \\
\hline sh human ZNF622\#1: atcggaaagtggagatgatgaa & This paper & $\mathrm{N} / \mathrm{A}$ \\
\hline sh human ZNF622\#2: tggagacgattgggaagatatt & This paper & $\mathrm{N} / \mathrm{A}$ \\
\hline sh mouse Znf622\#1: agagaaagttggtgttggcaaa & This paper & $\mathrm{N} / \mathrm{A}$ \\
\hline sh mouse Znf622\#2: tgtgacagttgctaggaatcaa & This paper & $\mathrm{N} / \mathrm{A}$ \\
\hline $\begin{array}{l}\text { Oligonucleotides for mouse genotyping and } \\
\text { real-time qPCR see Table S2 }\end{array}$ & This paper & N/A \\
\hline \multicolumn{3}{|l|}{ Recombinant DNA } \\
\hline pCMV-HA-HectD1 WT & Sarkar and Zohn, 2012 & $\mathrm{~N} / \mathrm{A}$ \\
\hline pCMV-HA-HectD1 C2579G & Sarkar and Zohn, 2012 & $\mathrm{~N} / \mathrm{A}$ \\
\hline pCMV-HA-HectD1 truncates/deletion mutants & This paper & $\mathrm{N} / \mathrm{A}$ \\
\hline pOZ-FH-ZNF622 full length & This paper & $\mathrm{N} / \mathrm{A}$ \\
\hline
\end{tabular}




\begin{tabular}{|l|l|l|}
\hline pOZ-FH-ZNF622 truncates/deletion mutants & This paper & N/A \\
\hline pOZ-Flag-ZNF622 & This paper & N/A \\
\hline Softwares and Algorithms & \multicolumn{2}{|l|}{} \\
\hline Flowjo & $\begin{array}{l}\text { https://www.flowjo.com/soluti } \\
\text { ons/flowjo }\end{array}$ & RRID:SCR_008520 \\
\hline GraphPad Prism & http://www.graphpad.com/ & RRID:SCR_002798 \\
\hline Fiji & http://fiji.sc & RRID:SCR_002285 \\
\hline QuantStudio Real-Time PCR software & Thermo Fisher & $\begin{array}{l}\text { https://www.thermofisher.com/us/en/home/life- } \\
\text { science/pcr/real-time-pcr/ }\end{array}$ \\
\hline SAINT & Choi et al., 2012 & $\begin{array}{l}\text { http://saint-apms.sourceforge.net/ } \\
\text { Main.html }\end{array}$ \\
\hline ELDA & Hu and Smyth, 2009 & http://bioinf.wehi.edu.au/software/elda// \\
\hline Sequest & Eng et al., 1994 & $\begin{array}{l}\text { http://tools.thermofisher.com/content/sfs/manu } \\
\text { als/Man-XCALI-97160-SEQUEST-331-User- } \\
\text { ManXCALI97160-B-EN.pdf }\end{array}$ \\
\hline
\end{tabular}

\section{RESOURCE AVAILABILITY}

\section{Lead Contact}

Further information and requests for resources and reagents should be directed to and will be fulfilled by the Lead Contact, Wei Tong (tongw@,chop.edu).

\section{Materials Availability}

This study did not generate new unique reagents.

\section{Data and Code Availability}

This study did not generate new datasets.

\section{EXPERIMENTAL MODEL AND SUBJECT DETAILS}

\section{Mouse models}

Hectdl "knockout-first, conditional-ready" mouse line (C57B6/NGpt-Hectd1 1 ${ }^{\text {tmla }} / \mathrm{Gpt}$, \#T000502) was obtained from EuComm (the ES lines were generated by European Consortium) and Model Animal Resource Information Platform of Nanjing University, China. FLP1 recombinase transgenic mice (B6;SJL-Tg(ACTFLPe)9205Dym/J, \#003800) and SJL (CD45.1) recipient mice were purchased from the Jackson Laboratory (Rodriguez et al., 2000). Vav1-Cre mice were originally generated by Dr. Thomas Graf (Stadtfeld and Graf, 2005) and generously provided by Dr. Nancy Speck (Chen et al., 2009). Hectdl transgenic mice were first crossed with RosaFlippase mice to eliminate FRT-flanked LacZ and Neo mini-gene, and then with wild type mice to get rid of the Rosa-Flippase gene to minimize the possible effects of these elements in hematopoiesis. The resultant mice with loxP-flanked Hectdl alleles (Hectdl lff) targeting exon 3 were crossed with Vav-cre transgenic mice to obtain the control Hectdl fff and Hectdl $I^{\text {ff; }}$ Vav conditional knockout mice (cKO). All mice were bred and grown in house in pathogen-free animal facilities. Both male and female mice (8 12 weeks old) were used and randomly assigned for all experiments. All the animal studies were performed under an approved protocol by the Institutional Animal Care and Use committee of the Children's Hospital of Philadelphia.

For 5-FU challenge, sex and age-matched (8 12 weeks old) adult Hectdl $I^{f f f}$ and Hectd $I^{f f ;}$;Vav mice were injected intraperitoneally with 5-Fluorouracil (5-FU, Cat\#F6627, Sigma, 150mg/kg body 
weight, pH8.5 in PBS) (Rozenova et al., 2015). Total BM cell count, HSC frequency, cell cycle and apoptosis assay were determined after 10 days of 5-FU administration. For Cy/GCSF induced stress, we intraperitoneally injected mice with 1 dose of cyclophosphamide (4mg/mouse, Baxer) followed by two daily subcutaneous injections of 5ug GCSF (Neupogen, Amgen). 1 day after the last GCSF injection, mice were euthanized for subsequent analysis (Morrison et al., 1997; Signer et al., 2014).

\section{Cell lines}

TF-1 cell lines were purchased from American Type Culture Collection (ATCC) and grown in RPMI-1640 media supplemented with 10\% bovine calf serum (Cat\# SH30072.03, HyClone), 2mM L-glutamine (Cat\# 25030-081, Gibco) and penicillin/Streptomycin (Gibco) and 2ng/mL GM-CSF (PeproTech) at $37^{\circ} \mathrm{C}$ and $95 \%$ humidity in an atmosphere of $5 \% \mathrm{CO}_{2} .293 \mathrm{~T}$ cells were from ATCC and grown in DMEM media supplemented with $10 \%$ bovine calf serum, $2 \mathrm{mM}$ L-glutamine and penicillin/Streptomycin at $37^{\circ} \mathrm{C}$ and $95 \%$ humidity in an atmosphere of $5 \% \mathrm{CO}_{2}$. BaF3 cells were maintained in RPMI-1640 media supplemented with $10 \%$ bovine calf serum and 10\% WEHI supernatant at $37^{\circ} \mathrm{C}$ and $95 \%$ humidity in an atmosphere of $5 \% \mathrm{CO}_{2}$.

\section{METHOD DETAILS}

\section{Genotyping and qPCR}

Mouse tail genomic DNAs were isolated with the standard proteinase K lysis protocol. Genotyping was performed by PCR. To evaluated Vav-cre excision efficiency in hematopoietic stem and progenitor cells, we plated total BM cells into M3434 methylcellulose semisolid media (StemCell Technologies Inc) for colony formation. Single colonies were picked, resuspended in 90uL buffer (50mM NaOH, 0.2mM EDTA), and boiled for 20min, followed by mixing with $10 \mathrm{uL} 1 \mathrm{M}$ Tris, pH8.0 for neutralization. The lysates were subsequently used for genotyping. Sequences of genotyping primers are listed in Table S2.

For quantitative real time PCR assay, total RNA was extracted using RNeasy Mini Kit (Qiagen). Reverse-transcription reaction was performed with random primers using qScript cDNA Supermix (Quanta Biosciences), and quantitative PCR was done with SYBR Green Master Mix (Applied Biosystems). Sequences of qPCR primers are listed in Table S2.

\section{Constructs and virus packaging}

pCMV-HA-HectD1 WT or C2579G mutant constructs were kindly gifted by Dr. Irene E. Zohn (Children's Research Institute, United States, Washington, DC, United States) (Sarkar and Zohn, 2012). The truncation mutants were amplified by PCR and subcloned into pCMV-HA vector. Human ZNF622 cDNA was amplified by PCR from a homemade cDNA library from TF-1 cells and constructed into a retroviral pOZ-FH vector that contains a Flag and a HA tag. All the deletion or truncation mutants were subcloned into the pOZ-FH vector. HectD1 or ZNF622 miR30-based shRNA constructs were subcloned into Lentiviral vector (pCL20.MSCV.mir30.PGK.mCherry) generously provided by Dr. Shannon McKinney-Freeman

\section{Complete blood count (CBC), flow cytometry of HSPCs and lineage cells}

Peripheral blood was collected from 8 12 week-old Hectdl ${ }^{f / f}$ and Hectdl ${ }^{f f ; \text {; Vav }}$ mice into EDTAcoated tubes. CBC analysis was performed with a Hemavet 950 (Drew Scientific, Inc). For lineage staining, cells from peripheral blood were lysed with RBC lysis buffer $\left(0.8 \% \mathrm{NH}_{4} \mathrm{Cl}, 10 \mathrm{uM}\right.$ EDTA, $\mathrm{pH} 7.4 \sim 7.6)$ for $10 \mathrm{~min}$ at $4^{\circ} \mathrm{C}$ to remove red blood cells, followed by staining with different fluorochrome-conjugated anti-Gr-1 (RB6-8C5) (granulocytes), -Mac1 (M1/70) (macrophages), - 
B220 (RA3-6B2) (mature B cells), -CD4 (GK1.5) and -CD8 (53-6.7) (T cells) antibodies, for $30 \mathrm{~min}$ at $4^{\circ} \mathrm{C}$. After washing with flow buffer (PBS containing $0.5 \% \mathrm{BSA}$ ), cells were suspended in flow buffer containing $1 \mathrm{ug} / \mathrm{mL}$ propidium iodide $(\mathrm{PI})$ or $2.5 \mathrm{ug} / \mathrm{mL}$ DAPI for flow cytometry analysis.

HSPCs staining was conducted as described previously (Lv et al., 2017). Cells from BM (2 femurs +2 tibias +2 hips for one mouse) or spleen were harvested in PBS containing $0.5 \%$ BSA, and quickly lysed with RBC lysis buffer for $1 \mathrm{~min}$ at $4^{\circ} \mathrm{C}$. Cells were then stained with biotinconjugated anti-Gr-1 (RB6-8C5), -Mac1 (M1/70), -B220 (RA3-6B2), -CD19 (eBio1D3), -Ter119 (TER-119), -CD5 (53-7.3), -CD4 (GK1.5), -CD8 (53-6.7), in combination with APC-Cy7-c-Kit (2B8), PerCP-Cy5.5-Sca1 (E13-161.7 or D7), FITC-CD48 (HM48-1), PE-Cy7-CD150 (TC1512F12.2), APC-CD34 (RAM34), and PE-Flk2 (A2F10.1) antibodies for 30min on ice, followed by secondary staining with streptavidin-PE-TexasRed (Invitrogen SA1017, 1:50) for 30min on ice. Different HSPC subpopulations were defined as long-term stem cells (LT-HSCs, in $^{-} \mathrm{Scal}^{+} \mathrm{c}-$ $\mathrm{Kit}^{+} \mathrm{Flk} 2^{-} \mathrm{CD} 150^{+} \mathrm{CD} 48^{-}$), short-term stem cells (ST-HSCs, Flk2-CD150-CD48- LSK), multiple potent progenitors (megakaryocyte/erythroid-biased MPP2, Flk2-CD150 ${ }^{+} \mathrm{CD} 48^{+}$LSK; myeloidbiased MPP3, Flk2-CD150-CD48 ${ }^{+}$LSK; lymphoid-biased MPP4, Flk2 ${ }^{+}$CD150-LSK) (Pietras et al., 2015).

For committed progenitor cell staining, namely granulocyte-monocyte progenitor (GMP, $\mathrm{CD}^{+} 4^{+} \mathrm{CD} 16 / 32^{+} \mathrm{Lin}^{-} \mathrm{c}-\mathrm{Kit}^{+} \mathrm{Sca}{ }^{-}$) cells, common myeloid progenitor (CMP, CD34 ${ }^{+} \mathrm{CD} 16 / 32^{-}$ $\mathrm{LKS}^{-}$) cells and megakaryocyte-erythrocyte progenitor (MEP, CD34 ${ }^{+} \mathrm{CD} 16 / 32^{-} \mathrm{LKS}^{-}$), cells from the BM or spleen were stained with PE-FrRIII/II (CD16/CD32) for 30min on ice after a quick RBC lysis, followed by blocking with rat serum, then stained with biotin-conjugated lineage panel as described above, along with APC-Cy7-c-Kit (2B8), PerCP-cy5.5-Scal (E13-161.7 or D7), APC-CD34 for $1 \mathrm{~h}$ on ice.

Lineage cell FACS samples were analyzed on a BD FACS Canto flow cytometer, while HSPC and progenitor samples were analyzed on a BD FACS Fortessa flow cytometer. Data were analyzed on FlowJo (FlowJo, LLC).

\section{Competitive BMT and limiting dilution BMT}

For competitive BMT, 1 million total BM cells from 8 12-week-old Hectdl ${ }^{\text {flf }}$ or Hectdl Iff; Vav $^{\text {B }}$ (CD45.2) mice were mixed with the same number of competitor total BM cells (CD45.1), and transplanted into lethally irradiated (a split dose of 10Gy) recipient mice (CD45.1/2) by retroorbital injection. Every four weeks after BMT, donor cell reconstitution in periphery blood (PB) was evaluated by flow cytometry. 16 weeks after BMT, reconstituted donor stem and progenitor cells (HSPCs) from BM or spleen were analyzed by flow cytometry.

For limiting dilution BMT, an increasing number $(10 \mathrm{k}, 30 \mathrm{k}, 100 \mathrm{k})$ of total BM cells from Hectdl fff or Hectdl $1^{f l f ; V a v}(C D 45.2)$ mice were mixed with a fixed number $(300 \mathrm{k})$ of competitor BM cells and transplanted into lethally irradiated recipient mice (Bersenev et al., 2008). 16 weeks after BMT, donor cell percentage in the PB was evaluated by flow cytometry. Mice with more than $1 \%$ of donor-derived cells were defined as "positive". Data were analyzed by ELDA (Hu and Smyth, 2009).

\section{HSC sorting and transplantation}

HSC purification and BMT were performed as described previously (Balcerek et al., 2018). Lineage positive cells were first depleted using a lineage cell depletion kit (Cat\# 130-090-858, Miltenyi Biotec). Lineage negative (Lin-) cells were then stained with APC-Cy7-c-Kit (2B8), 
PerCP-Cy5.5-Sca1 (E13-161.7 or D7), FITC-CD48 (HM48-1), PE-Cy7-CD150 (TC15-12F12.2). LT-HSCs were purified with MoFlo Astrios Sorter and 100 LT-HSCs were seeded in a roundbottom 96-well plate. These LT-HSCs were either transplanted on the day (D0 BMT) or cultured in SFEM media supplemented with $100 \mathrm{ng} / \mathrm{mL} \mathrm{SCF}$ and $20 \mathrm{ng} / \mathrm{mL}$ TPO for 12 days, and all the resultant cells were then transplanted (Day12 BMT). Specifically, 100 LT-HSCs (D0 BMT) or 100 LT-HSCs-derived cells (CD45.2) at day12 (D12 BMT) were mixed with 500k Sca1-depleted competitor BM cells (CD45.1 or CD45.1/2) and injected retro-orbically into lethally irradiated (10Gy) recipient mice (CD45.1/2 or CD45.1). Every four weeks after BMT, donor cell reconstitution in the PB was evaluated by flow cytometry. 16 weeks after BMT, reconstituted donor stem and progenitor cells (HSPCs) from BM or spleen were analyzed by flow cytometry.

\section{OP-Puro (O-propargyl-puromycin) Click-iT protein synthesis assay}

To detect protein synthesis rate in vivo, primary or stressed mice were injected intraperitoneally with OP-Puro (Cat\# HY-15680, MCE; 50mg/kg body weight, pH6.4-6.6 in PBS) for 1 hour before euthanasia (Signer et al., 2014). Total BM cells were harvested and live stained with cell surface markers for HSCs/MPPs after a quick RBC lysis. Cells were then fixed with BD Cytofix solution for 20min on ice. After washing with BD Perm/Wash buffer, cells were permeabilized with BD Cytoperm Plus solution for 10min on ice, followed by refixing in Cytofix solution for $5 \mathrm{~min}$. The azide-alkyne reaction was performed using Click-iT plus OPP Alexa Fluor 647 or 488 kit (Cat\# $\mathrm{C} 10458$, Invitrogen) for 30min at room temperature. Cells were then washed and resuspended in flow buffer, and analyzed by on a BD FACS Fortessa flow cytometer.

For ex vivo analysis, OP-Puro was added to cell culture at the final concentration of $20 \mathrm{uM}$ for 1 hour at a $37^{\circ} \mathrm{C}$ incubator. The azide-alkyne reaction was performed as described above.

\section{Cell cycle and cell apoptosis assay}

For BrdU cell cycle analysis, mice were injected with 200uL BrdU (10mg/mL, Cat\# 550891, BD Pharmingen) for 2 hours. Total BM cells were stained with cell surface markers for HSCs/MPPs, and then fixed and permeabilized with BD Cytofix/Cytoperm kit, followed by treatment with $300 \mathrm{ug} / \mathrm{mL}$ DNaseI for 1 hours at a $37^{\circ} \mathrm{C}$ water bath. After washing with BD Perm/Wash buffer, cells were stained with FITC-anti-BrdU (Cat\# 5133284, BD Pharmingen) for 20min at room temperature. After washing, cells were resuspended in flow buffer with DAPI $(5 \mathrm{ug} / \mathrm{mL})$, and analyzed by flow cytometry.

For apoptosis assay, total BM cells were stained with cell surface markers for HSCs/MPPs. After washing with flow buffer, cells were resuspended in 200uL Annexin V binding buffer. 10uL FITCAnnexin V (Cat\# 55647, BD Pharmingen) and DAPI were added for 15min at room temperature in the dark, followed by adding $800 \mathrm{uL}$ binding buffer. Samples were analyzed on a BD Fortessa cytometer within 1 hour.

\section{Viral transduction of LSK cells and transplantation}

For LSK lentiviral infection and rescue BMT, sorted LSK cells from either Hectdl ${ }^{\text {flf }}$ or Hectdl $1^{f f ;}$; Vav (CD45.2) mice were cultured in SFEM media (StemCell Technologies Inc) supplemented with 10\% FBS (SAFC Biosciences) and cytokines (100 ng/mL mSCF, $20 \mathrm{ng} / \mathrm{mL} \mathrm{mTpo}, 20 \mathrm{ng} / \mathrm{mL}$ FLT3L, $20 \mathrm{ng} / \mathrm{mL}$ IL6) for 2 days. Lentivirus carrying mCherry/shLuc or mCherry/shmZNF622 were preloaded twice into a RetroNectin (T100B, Takara)-coated 12-well plate (Modlich et al., 2009). Cultured LSKs were transferred to the lentivirus-preload plates and incubated for one more day. At day3, 250k cultured LSKs were mixed with 500k Scal-depleted competitor BM cells and injected into lethally-irradiated recipient mice. A small fraction of infected cells was spared for flow cytometry to evaluate the viral infection efficiency (Jiang et al., 2012). 


\section{Immunoprecipitation (IP)}

For each anti-HA immunoprecipitation, ten $\sim 80 \%$ confluent $10 \mathrm{~cm}$ dishes of $293 \mathrm{~T}$ cells were transiently transfected with pCMV-HA empty vector (control) or pCMV-HA-HectD1. 48 hours after transfection, cells were lysed with IP buffer (10mM Tris, $\mathrm{pH} 7.4,150 \mathrm{mM} \mathrm{NaCl}, 0.5 \%$ NP-40, $1 \mathrm{mM} \mathrm{NaF}, 1 \mathrm{mM} \mathrm{Na}_{3} \mathrm{VO}_{4}$, PMSF, protease inhibitor cocktail, $10 \mu \mathrm{M}$ PR-619 (LifeSensors), $4 \mathrm{mM}$ 1,10-phenanthroline (o-PA; Mallinckrodt Chemicals), 4mM N ethylmaleimide (NEM; SigmaAldrich)) for $30 \mathrm{~min}$ at $4^{\circ} \mathrm{C}$. Cell lysates were clarified by centrifugation at 13, $000 \mathrm{rpm}$ for $10 \mathrm{~min}$ at $4^{\circ} \mathrm{C}$, and then pre-cleared with protein $\mathrm{A} / \mathrm{G}$ beads for $30 \mathrm{~min}$.

HA-EZ Agarose beads (E6779, Sigma) were prepared by being sequentially washed with $0.1 \mathrm{M}$ pH2.5 Glycine, twice in 1M pH8.0 Tris buffer, and twice in IP buffer. Precleared supernatants were incubated with $100 \mathrm{uL}$ washed HA-EZ Agarose beads for $4 \mathrm{hrs}$ with gentle agitation. We transferred the IPs to BioRad Micro Bio-Spin Chromatography Columns (Cat\# 732-6204), and washed columns with $1 \mathrm{~mL}$ IP buffer for four times, followed by a quick spin down to drain the leftover IP buffer. We then seal the bottom of the columns with parafilm, and added $50 \mathrm{uL} 1 \mathrm{mg} / \mathrm{mL}$ HA peptides (\#26184, Thermo Fisher Scientific) for $15 \mathrm{~min}$ at $30^{\circ} \mathrm{C}$ with occasional mixing, and collected the elute as "HA elute1". This step was repeated to get another 50uL HA peptide eluate as "HA elute2". Then the beads were incubated with $50 \mathrm{uL}$ of $0.1 \mathrm{M} \mathrm{pH} 2.5$ Glycine for 5 min at room temperature twice to get "Glycine elute 1 and 2", which was neutralized with $5 \mathrm{uL}$ pH8.0 Tris. Lastly, the beads were boiled in $75 \mathrm{uL} 1 * \mathrm{LDS}$ loading buffer. All elutes from above were added with $25 \mathrm{uL} 3 *$ LDS loading buffer and boiled for $5 \mathrm{~min}$. A small aliquot of eluted samples was resolved with SDS-PAGE, and evaluated by silver staining. Once determined the purification was successful, we loaded majority of the first HA eluates on an SDS-PAGE, stained with colloidal blue. Gel slices were excised and subsequently subjected to mass spectrometry analysis at the Harvard Taplin Mass Spectrometry facility.

\section{Mass spectrometry and Significance analysis of INTeractome (SAINT)}

Excised gel bands were cut into approximately $1 \mathrm{~mm}^{3}$ pieces. Gel pieces were then subjected to a modified in-gel trypsin digestion procedure (Shevchenko et al., 1996). Gel pieces were washed and dehydrated with acetonitrile for $10 \mathrm{~min}$, followed by removal of acetonitrile. Pieces were then completely dried in a speed-vac. Rehydration of the gel pieces was with $50 \mathrm{mM}$ ammonium bicarbonate solution containing $12.5 \mathrm{ng} / \mu 1$ modified sequencing-grade trypsin (Promega, Madison, WI) at $4^{\circ} \mathrm{C}$. After $45 \mathrm{~min}$, the excess trypsin solution was removed and replaced with $50 \mathrm{mM}$ ammonium bicarbonate solution to just cover the gel pieces. Samples were then placed in a $37^{\circ} \mathrm{C}$ room overnight. Peptides were later extracted by removing the ammonium bicarbonate solution, followed by one wash with a solution containing $50 \%$ acetonitrile and $1 \%$ formic acid. The extracts were then dried in a speed-vac $(\sim 1 \mathrm{hr})$. The samples were then stored at $4^{\circ} \mathrm{C}$ until analysis.

On the day of analysis, the samples were reconstituted in 5-10 $\mu 1$ of HPLC solvent A $(2.5 \%$ acetonitrile, $0.1 \%$ formic acid). A nano-scale reverse-phase HPLC capillary column was created by packing $2.6 \mu \mathrm{m} \mathrm{C} 18$ spherical silica beads into a fused silica capillary (100 $\mu \mathrm{m}$ inner diameter $\mathrm{x} \sim 30 \mathrm{~cm}$ length) with a flame-drawn tip (Peng and Gygi, 2001). After equilibrating the column each sample was loaded via a Famos auto sampler (LC Packings, San Francisco CA) onto the column. A gradient was formed and peptides were eluted with increasing concentrations of solvent B (97.5\% acetonitrile, $0.1 \%$ formic acid).

Eluted peptides were subjected to electrospray ionization and then entered into an LTQ Orbitrap Velos Pro ion-trap mass spectrometer (Thermo Fisher Scientific, Waltham, MA). Peptides were detected, isolated, and fragmented to produce a tandem mass spectrum of specific fragment ions 
for each peptide. Peptide sequences (and hence protein identity) were determined by matching protein databases with the acquired fragmentation pattern by the software program, Sequest (Thermo Fisher Scientific, Waltham, MA) (Eng et al., 1994). All databases include a reversed version of all the sequences and the data was filtered to between a one and two percent peptide false discovery rate.

We performed 3 biological replicates of IP-MS. Three replicates were evaluated by CRAPome (v1.1) (Contaminant Repository for Affinity Purification) analysis (http://www.crapome.org/) (Mellacheruvu et al., 2013). We used five HA controls with four (CC51, CC52, CC53, CC54) from CRAPome repository and one from our own HA control. To calculate SAINT scores (Choi et al., 2012), spectral counts were analyzed using SAINT parameters "Lowmode $=0$, MinFold $=0$, Normalize $=1$ ". The presented SAINT score was the average probability of SAINT results from all three biological replicates. Fold change (FC) is the ratio of the normalized spectral counts of a potential HectD1-interactor to the average of three highest normalized spectral counts of that protein across the negative controls. We chose the cutoff of SAINT score $\geq 0.5$ and fold change (FC) $\geq 3$ based on the established HectD1-interactors (RIOK2, (Varjosalo et al., 2013); ZRANB1, (Tran et al., 2013); SMC2, (Li et al., 2015)). ZNF622 was among the top hits of all 3 replicates (Supplemental Excel). Gene Ontology (GO) analysis of HectD1-interacting proteins was performed using PANTHER Classification System (http://pantherdb.org) (Mi et al., 2019).

\section{Polysome profiling}

20 30 million TF-1 cells with shLuc or shHECTD1 were pre-treated with $100 \mathrm{ug} / \mathrm{mL}$ cycloheximide $(\mathrm{CHX})$ for $5 \mathrm{~min}$ and washed with ice-cold $\mathrm{CHX}$-containing PBS. After centrifugation, cell pellets were lysed in polysome lysis buffer $(20 \mathrm{mM}$ Tris, $\mathrm{pH} 7.5,1.5 \mathrm{mM} \mathrm{MgCl}$,

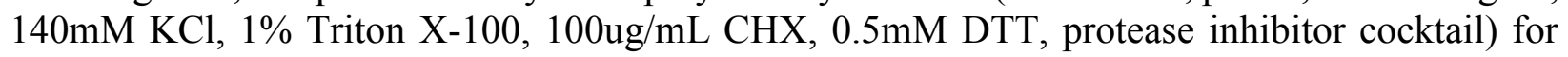
$10 \mathrm{~min}$ in ice with gentle rocking. The cell lysate was clarified by centrifugation at $17,000 \mathrm{~g}$ for $10 \mathrm{~min}$ at $4{ }^{\circ} \mathrm{C}$. $\mathrm{OD}_{260}$ value was measured in Nanodrop. Linear sucrose gradient $(7 \%-45 \%)$ was generated using a Gradient Maker (BioComp Instruments, Canada). 15-20 OD 260 of total cell extract was loaded on the sucrose gradient, followed by ultracentrifugation at 35,000rpm for $3 \mathrm{hrs}$ $20 \mathrm{~min}$ at $4^{\circ} \mathrm{C}$ in SW40 rotor. Polysome profiling was analyzed with a BioComp fractionator.

For detection of protein distribution, a total of 13 fractions $(830 \mathrm{uL} /$ fraction) from polysome profiling were collected by a fraction collector (Cat\# 4422151, FC-203B, Gilson). To extract proteins for Western Blot (WB) analysis, 150uL of each fraction was pelleted by methanolcholoform $-\mathrm{H}_{2} \mathrm{O}$ precipitation with sequential addition of $600 \mathrm{uL}$ ice-cold methanol, $225 \mathrm{uL}$ chloroform and $450 \mathrm{uL} \mathrm{H}_{2} \mathrm{O}$. The reaction was thoroughly mixed by inversion and centrifuged at $20,000 \mathrm{~g}$ for $4 \mathrm{~min}$ at $4^{\circ} \mathrm{C}$. After carefully removing the aqueous layer, $1 \mathrm{~mL}$ prechilled methanol was added and mixed by inversion, followed by centrifugation at $20,000 \mathrm{~g}$ for $4 \mathrm{~min}$ at $4{ }^{\circ} \mathrm{C}$. The supernatant was then decanted, and the protein pellet was dried at room temperature. 50uL $1 *$ LDS loading solution was directly added to dissolve protein pellets by frequent pipetting up and down. Due to the extra abundance of proteins in cytoplasmic fractions 1-3, 2uL of fractions 1-3 along with $20 \mathrm{uL}$ of fractions 4-13 (different ribosomal fractions) were loaded onto an SDS-PAGE for WB analysis.

\section{Ribosomal subunit dissociation and re-association assay}

This assay was adapted from a previously published work (Burwick et al., 2012). For ribosomal subunit dissociation, 20 30 TF-1 cells were harvested without cycloheximide treatment and lysed with low $\mathrm{Mg}^{2+}$ buffer (20mM Tris, pH7.4, $140 \mathrm{mM} \mathrm{KCl,} 0.25 \mathrm{mM} \mathrm{MgCl}_{2}, 0.5 \mathrm{mM}$ DTT, $1 \%$ Triton X-100, EDTA-free protease inhibitor cocktail (Roche)) for 10min on ice. After clarification by centrifugation at $17,000 \mathrm{~g}$ for $5 \mathrm{~min}$ at $4^{\circ} \mathrm{C}$, the cell lysate was divided into two aliquots. One aliquot 
was loaded on a $7-45 \%$ low $\mathrm{Mg}^{2+}$ sucrose gradient $(20 \mathrm{mM}$ Tris, $\mathrm{pH} 7.4,140 \mathrm{mM} \mathrm{KCl}, 0.25 \mathrm{mM}$ $\mathrm{MgCl}_{2}, 0.5 \mathrm{mM}$ DTT, EDTA-free protease inhibitor cocktail (Roche)) and analyzed with a BioComp fractionator to detect total $40 \mathrm{~S}$ and $60 \mathrm{~S}$.

For ribosomal subunit re-association, $2.5 \mathrm{M} \mathrm{MgCl} 2$ was added to the other aliquot for a final concentration of $10 \mathrm{mM} \mathrm{Mg}^{2+}$ and incubated for $5 \mathrm{~min}$ at $37^{\circ} \mathrm{C}$. The resultant cell lysate was loaded on a 7-45\% high $\mathrm{Mg}^{2+}$ sucrose gradient (20mM Tris, $\mathrm{pH} 7.4,140 \mathrm{mM} \mathrm{KCl}, 10 \mathrm{mM} \mathrm{MgCl} 2,0.5 \mathrm{mM}$ DTT, EDTA-free protease inhibitor cocktail (Roche)) and analyzed with a BioComp fractionator.

\section{Denatured His-ubiquitination assay}

293T cells were transfected with indicated HectD1 and ZNF622 constructs, as well as His-tagged $\mathrm{Ub}$ WT, K48R or K63R mutants. 2 days later, cells were harvested and washed with cold PBS twice, and then lysed with denatured Urea Lysis buffer $\left(100 \mathrm{mM} \mathrm{Na}_{2} \mathrm{HPO}_{4}, 100 \mathrm{mM} \mathrm{NaH}_{2} \mathrm{PO}_{4}\right.$, $10 \mathrm{mM}$ Tris, $\mathrm{pH} 8.0,0.2 \%$ Triton X-100, $5 \mathrm{mM} \beta$-Mercaptoethanol, $10 \mathrm{mM}$ Imidazole, $8 \mathrm{M}$ urea), followed by immediate vortex and rocking at RT for $20 \mathrm{~min}$. After centrifuging for $10 \mathrm{~min}$ at $15,000 \mathrm{~g}$, supernatant was obtained and incubated with HisPur Ni-NTA resin $(88221$ Thermo Scientific) for $2 \mathrm{hrs}$ at RT. Resin was then washed 3 times with wash buffer $\left(100 \mathrm{mM} \mathrm{Na}_{2} \mathrm{HPO}_{4}\right.$, $100 \mathrm{mM} \mathrm{NaH} 2 \mathrm{PO}_{4}, 10 \mathrm{mM}$ Tris, pH6.3, 0.2\% Triton X-100, 5mM $\beta$-Mercaptoethanol, $20 \mathrm{mM}$ Imidazole, $8 \mathrm{M}$ urea). Bound ubiquitinated proteins were eluted with SDS elution buffer $(200 \mathrm{mM}$ Imidazole, 5\% SDS, 150mM Tris, pH6.8, 30\% glycerol, $720 \mathrm{mM} \beta$-Mercaptoethanol) and subjected to western blot analysis.

\section{Cytokine signaling, protein half-life assay and Western blot (WB)}

For cytokine signaling, TF-1/MPL cells were stared in RPMI-1640 media plus $0.5 \%$ BSA for 2$4 \mathrm{hrs}$, and then stimulated with TPO for indicated time points and snap-frozen in dry ice. For mTOR signaling, we also stimulated starved cells with a graded concentration of calf serum. Cell pellets were lysed in LDS loading buffer and sonicated for homogenization. For measuring protein halflife, cycloheximide (CHX) was employed to block de novo protein synthesis for different time points prior to cell harvest.

Protein lysates were subjected to standard WB protocols. Briefly, samples were resolved by SDSPAGE, and transferred to NC membrane. For all primary phosphor-antibody blots, membranes were blocked with 5\% BSA (BP1600-100, Fisher Bioreagents) in TBS-T, while other primary antibody blots were blocked with 5\% non-fat milk (sc2325, Santa Cruz). Membranes were incubated with primary antibodies for $2 \mathrm{hrs}$ at room temperature or overnight in cold room. Following primary antibody blots, membranes were washed with TBS-T extensively, and then incubated with HRP-conjugated secondary antibody for $1 \mathrm{hr}$ at room temperature. After extensive washing, membranes were developed with ECL (\#34095, Thermo Scientific). To compare immunoblots with a large number of samples that require multiple gels, samples were resolved in SDS-PAGE gels in parallel. Immunoblots were processed and developed side by side, then images were placed side-by-side for presentation.

\section{HSC cell growth and MTT proliferation assay}

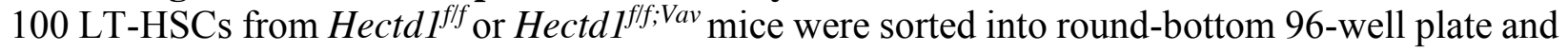
cultured in SFEM (StemCell Technologies Inc.) supplemented with 10\% FBS and various combinations or concentrations of cytokines as indicated in the main text. At different days, cell numbers were enumerated in the presence of trypan blue using a hemacytometer slide.

TF-1/MPL cells with shLuc or shHECTD1 were cultured in an increasing dose of GM-CSF or TPO in a 96-well plate (10k cells/100uL per well) for 3 days. 3-(4,5-dimethylthiazole-2-yl)-2,5- 
diphenyl tetrazolium bromide (MTT; M6494, Invitrogen) was added to media at a final concentration of $0.5 \mathrm{mg} / \mathrm{mL}$ for $3-4 \mathrm{hrs}$ at $37^{\circ} \mathrm{C}$. Stopping buffer (15\% SDS, $2.5 \%$ Acetic acid, $50 \%$ dimethylformamide) was then added to terminate the reaction. The absorbance was read by a spectrophotometer at $570 \mathrm{~nm}$.

\section{QUANTIFICATION AND STATISTICAL ANALYSIS}

Statistics for MTT, HSC cell growth and CFC assays were performed using unpaired two-tailed Student's $t$ test and error bars indicate mean \pm SD. Signal intensities of Western blot bands were quantified by Fiji software. Statistics was analyzed using unpaired two-tailed Student's $t$ test. All the relevant quantifications performed in primary mice or transplanted mice were analyzed by GraphPad Prism and error bars indicate mean \pm SEM. Paired $t$ test was used for pairwise comparison of HSC/MPP numbers in Cyclophosphamide+2GCSF challenged mice. Details of " $n$ " values describing the number of experiment repeats or mice are shown in the figure legends. Statistics significance was determined by Student's $t$ test. ns, not significant; *: $p<0.05 ; * *: \mathrm{p}<0.01$; $* * *: \mathrm{p}<0.001$. 


\section{REFERENCE}

Aleidi, S.M., Yang, A., Sharpe, L.J., Rao, G., Cochran, B.J., Rye, K.A., Kockx, M., Brown, A.J., and Gelissen, I.C. (2018). The E3 ubiquitin ligase, HECTD1, is involved in ABCA1-mediated cholesterol export from macrophages. Biochim Biophys Acta Mol Cell Biol Lipids 1863, 359368.

Balcerek, J., Jiang, J., Li, Y., Jiang, Q., Holdreith, N., Singh, B., Chandra, V., Lv, K., Ren, J.G., Rozenova, K., et al. (2018). Lnk/Sh2b3 deficiency restores hematopoietic stem cell function and genome integrity in Fancd2 deficient Fanconi anemia. Nat Commun 9, 3915.

Barilari, M., Bonfils, G., Treins, C., Koka, V., De Villeneuve, D., Fabrega, S., and Pende, M. (2017). ZRF1 is a novel S6 kinase substrate that drives the senescence programme. EMBO J 36, 736-750.

Bersenev, A., Wu, C., Balcerek, J., and Tong, W. (2008). Lnk controls mouse hematopoietic stem cell self-renewal and quiescence through direct interactions with JAK2. J Clin Invest 118, 2832-2844.

Boocock, G.R., Morrison, J.A., Popovic, M., Richards, N., Ellis, L., Durie, P.R., and Rommens, J.M. (2003). Mutations in SBDS are associated with Shwachman-Diamond syndrome. Nat Genet 33, 97-101.

Burwick, N., Coats, S.A., Nakamura, T., and Shimamura, A. (2012). Impaired ribosomal subunit association in Shwachman-Diamond syndrome. Blood 120, 5143-5152.

Chauvin, C., Koka, V., Nouschi, A., Mieulet, V., Hoareau-Aveilla, C., Dreazen, A., Cagnard, N., Carpentier, W., Kiss, T., Meyuhas, O., et al. (2014). Ribosomal protein S6 kinase activity controls the ribosome biogenesis transcriptional program. Oncogene 33, 474-483.

Chen, M.J., Yokomizo, T., Zeigler, B.M., Dzierzak, E., and Speck, N.A. (2009). Runx1 is required for the endothelial to haematopoietic cell transition but not thereafter. Nature 457, 887891.

Choi, H., Liu, G., Mellacheruvu, D., Tyers, M., Gingras, A.C., and Nesvizhskii, A.I. (2012). Analyzing protein-protein interactions from affinity purification-mass spectrometry data with SAINT. Curr Protoc Bioinformatics Chapter 8, Unit8 15.

D'Alonzo, D., Emch, F.H., Shen, X., Bruder, E., De Geyter, C., and Zhang, H. (2019). Hectd1 is essential for embryogenesis in mice. Gene Expr Patterns 34, 119064.

de la Cruz, J., Karbstein, K., and Woolford, J.L., Jr. (2015). Functions of ribosomal proteins in assembly of eukaryotic ribosomes in vivo. Annu Rev Biochem 84, 93-129.

Dhanraj, S., Matveev, A., Li, H., Lauhasurayotin, S., Jardine, L., Cada, M., Zlateska, B., Tailor, C.S., Zhou, J., Mendoza-Londono, R., et al. (2017). Biallelic mutations in DNAJC21 cause Shwachman-Diamond syndrome. Blood 129, 1557-1562.

Duhamel, S., Goyette, M.A., Thibault, M.P., Filion, D., Gaboury, L., and Cote, J.F. (2018). The E3 Ubiquitin Ligase HectD1 Suppresses EMT and Metastasis by Targeting the +TIP ACF7 for Degradation. Cell Rep 22, 1016-1030.

Eng, J.K., McCormack, A.L., and Yates, J.R. (1994). An approach to correlate tandem mass spectral data of peptides with amino acid sequences in a protein database. J Am Soc Mass Spectrom 5, 976-989.

Finch, A.J., Hilcenko, C., Basse, N., Drynan, L.F., Goyenechea, B., Menne, T.F., Gonzalez Fernandez, A., Simpson, P., D'Santos, C.S., Arends, M.J., et al. (2011). Uncoupling of GTP hydrolysis from eIF6 release on the ribosome causes Shwachman-Diamond syndrome. Genes Dev 25, 917-929.

Greber, B.J., Boehringer, D., Montellese, C., and Ban, N. (2012). Cryo-EM structures of Arx1 and maturation factors Reil and Jjj1 bound to the 60S ribosomal subunit. Nat Struct Mol Biol 19, 1228-1233. 
Greber, B.J., Gerhardy, S., Leitner, A., Leibundgut, M., Salem, M., Boehringer, D., Leulliot, N., Aebersold, R., Panse, V.G., and Ban, N. (2016). Insertion of the Biogenesis Factor Reil Probes the Ribosomal Tunnel during 60S Maturation. Cell 164, 91-102.

Holcik, M., and Sonenberg, N. (2005). Translational control in stress and apoptosis. Nat Rev Mol Cell Biol 6, 318-327.

Hu, Y., and Smyth, G.K. (2009). ELDA: extreme limiting dilution analysis for comparing depleted and enriched populations in stem cell and other assays. J Immunol Methods 347, 70-78. Jiang, J., Balcerek, J., Rozenova, K., Cheng, Y., Bersenev, A., Wu, C., Song, Y., and Tong, W. (2012). 14-3-3 regulates the LNK/JAK2 pathway in mouse hematopoietic stem and progenitor cells. J Clin Invest 122, 2079-2091.

Kargas, V., Castro-Hartmann, P., Escudero-Urquijo, N., Dent, K., Hilcenko, C., Sailer, C., Zisser, G., Marques-Carvalho, M.J., Pellegrino, S., Wawiorka, L., et al. (2019). Mechanism of completion of peptidyltransferase centre assembly in eukaryotes. Elife 8.

Kisly, I., Remme, J., and Tamm, T. (2019). Ribosomal protein eL24, involved in two intersubunit bridges, stimulates translation initiation and elongation. Nucleic Acids Res 47, 406420.

Klinge, S., and Woolford, J.L., Jr. (2019). Ribosome assembly coming into focus. Nat Rev Mol Cell Biol 20, 116-131.

Li, W., Hu, Y., Oh, S., Ma, Q., Merkurjev, D., Song, X., Zhou, X., Liu, Z., Tanasa, B., He, X., et al. (2015). Condensin I and II Complexes License Full Estrogen Receptor alpha-Dependent Enhancer Activation. Mol Cell 59, 188-202.

Liaud, N., Horlbeck, M.A., Gilbert, L.A., Gjoni, K., Weissman, J.S., and Cate, J.H.D. (2019). Cellular response to small molecules that selectively stall protein synthesis by the ribosome. PLoS Genet 15, e1008057.

Liu, J., Xu, Y., Stoleru, D., and Salic, A. (2012). Imaging protein synthesis in cells and tissues with an alkyne analog of puromycin. Proc Natl Acad Sci U S A 109, 413-418.

Lo, K.Y., Li, Z., Bussiere, C., Bresson, S., Marcotte, E.M., and Johnson, A.W. (2010). Defining the pathway of cytoplasmic maturation of the 60S ribosomal subunit. Mol Cell 39, 196-208. Lv, K., Jiang, J., Donaghy, R., Riling, C.R., Cheng, Y., Chandra, V., Rozenova, K., An, W., Mohapatra, B.C., Goetz, B.T., et al. (2017). CBL family E3 ubiquitin ligases control JAK2 ubiquitination and stability in hematopoietic stem cells and myeloid malignancies. Genes Dev 31, 1007-1023.

Ma, C., Wu, S., Li, N., Chen, Y., Yan, K., Li, Z., Zheng, L., Lei, J., Woolford, J.L., Jr., and Gao, N. (2017). Structural snapshot of cytoplasmic pre-60S ribosomal particles bound by Nmd3, Lsg1, Tif6 and Reh1. Nat Struct Mol Biol 24, 214-220.

Mellacheruvu, D., Wright, Z., Couzens, A.L., Lambert, J.P., St-Denis, N.A., Li, T., Miteva, Y.V., Hauri, S., Sardiu, M.E., Low, T.Y., et al. (2013). The CRAPome: a contaminant repository for affinity purification-mass spectrometry data. Nat Methods 10, 730-736.

Menne, T.F., Goyenechea, B., Sanchez-Puig, N., Wong, C.C., Tonkin, L.M., Ancliff, P.J., Brost, R.L., Costanzo, M., Boone, C., and Warren, A.J. (2007). The Shwachman-Bodian-Diamond syndrome protein mediates translational activation of ribosomes in yeast. Nat Genet 39, 486-495. Meyer, A.E., Hoover, L.A., and Craig, E.A. (2010). The cytosolic J-protein, Jjj1, and Rei1 function in the removal of the pre-60 S subunit factor Arx1. J Biol Chem 285, 961-968.

Mi, H., Muruganujan, A., Ebert, D., Huang, X., and Thomas, P.D. (2019). PANTHER version 14: more genomes, a new PANTHER GO-slim and improvements in enrichment analysis tools. Nucleic Acids Res 47, D419-D426.

Modlich, U., Schambach, A., Li, Z., and Schiedlmeier, B. (2009). Murine hematopoietic stem cell transduction using retroviral vectors. Methods Mol Biol 506, 23-31. 
Morrison, S.J., Wright, D.E., and Weissman, I.L. (1997). Cyclophosphamide/granulocyte colony-stimulating factor induces hematopoietic stem cells to proliferate prior to mobilization. Proc Natl Acad Sci U S A 94, 1908-1913.

Oguro, H., Ding, L., and Morrison, S.J. (2013). SLAM family markers resolve functionally distinct subpopulations of hematopoietic stem cells and multipotent progenitors. Cell Stem Cell 13, 102-116.

Peng, J., and Gygi, S.P. (2001). Proteomics: the move to mixtures. J Mass Spectrom 36, 1083 1091.

Pietras, E.M., Reynaud, D., Kang, Y.A., Carlin, D., Calero-Nieto, F.J., Leavitt, A.D., Stuart, J.M., Gottgens, B., and Passegue, E. (2015). Functionally Distinct Subsets of Lineage-Biased Multipotent Progenitors Control Blood Production in Normal and Regenerative Conditions. Cell Stem Cell 17, 35-46.

Rodriguez, C.I., Buchholz, F., Galloway, J., Sequerra, R., Kasper, J., Ayala, R., Stewart, A.F., and Dymecki, S.M. (2000). High-efficiency deleter mice show that FLPe is an alternative to CreloxP. Nat Genet 25, 139-140.

Rozenova, K., Jiang, J., Donaghy, R., Aressy, B., Greenberg, R.A., and Tong, W. (2015). MERIT40 deficiency expands hematopoietic stem cell pools by regulating thrombopoietin receptor signaling. Blood 125, 1730-1738.

Ruggero, D., and Shimamura, A. (2014). Marrow failure: a window into ribosome biology. Blood 124, 2784-2792.

Sarkar, A.A., Nuwayhid, S.J., Maynard, T., Ghandchi, F., Hill, J.T., Lamantia, A.S., and Zohn, I.E. (2014). Hectd1 is required for development of the junctional zone of the placenta. Dev Biol 392, 368-380.

Sarkar, A.A., and Zohn, I.E. (2012). Hectd1 regulates intracellular localization and secretion of Hsp90 to control cellular behavior of the cranial mesenchyme. J Cell Biol 196, 789-800.

Shembade, N., and Harhaj, E.W. (2015). Elucidating dynamic protein-protein interactions and ubiquitination in NF-kappaB signaling pathways. Methods Mol Biol 1280, 283-295.

Shevchenko, A., Wilm, M., Vorm, O., and Mann, M. (1996). Mass spectrometric sequencing of proteins silver-stained polyacrylamide gels. Anal Chem 68, 850-858.

Signer, R.A., Magee, J.A., Salic, A., and Morrison, S.J. (2014). Haematopoietic stem cells require a highly regulated protein synthesis rate. Nature 509, 49-54.

Signer, R.A., Qi, L., Zhao, Z., Thompson, D., Sigova, A.A., Fan, Z.P., DeMartino, G.N., Young, R.A., Sonenberg, N., and Morrison, S.J. (2016). The rate of protein synthesis in hematopoietic stem cells is limited partly by 4E-BPs. Genes Dev 30, 1698-1703.

Stadtfeld, M., and Graf, T. (2005). Assessing the role of hematopoietic plasticity for endothelial and hepatocyte development by non-invasive lineage tracing. Development 132, 203-213.

Sugrue, K.F., Sarkar, A.A., Leatherbury, L., and Zohn, I.E. (2019). The ubiquitin ligase HECTD1 promotes retinoic acid signaling required for development of the aortic arch. Dis Model Mech 12.

Sulima, S.O., Hofman, I.J.F., De Keersmaecker, K., and Dinman, J.D. (2017). How Ribosomes Translate Cancer. Cancer Discov 7, 1069-1087.

Tan, S., Kermasson, L., Hoslin, A., Jaako, P., Faille, A., Acevedo-Arozena, A., Lengline, E., Ranta, D., Poiree, M., Fenneteau, O., et al. (2019). EFL1 mutations impair eIF6 release to cause Shwachman-Diamond syndrome. Blood 134, 277-290.

Tran, H., Bustos, D., Yeh, R., Rubinfeld, B., Lam, C., Shriver, S., Zilberleyb, I., Lee, M.W., Phu, L., Sarkar, A.A., et al. (2013). HectD1 E3 ligase modifies adenomatous polyposis coli (APC) with polyubiquitin to promote the APC-axin interaction. J Biol Chem 288, 3753-3767.

Tummala, H., Walne, A.J., Williams, M., Bockett, N., Collopy, L., Cardoso, S., Ellison, A., Wynn, R., Leblanc, T., Fitzgibbon, J., et al. (2016). DNAJC21 Mutations Link a Cancer-Prone 
Bone Marrow Failure Syndrome to Corruption in 60S Ribosome Subunit Maturation. Am J Hum Genet 99, 115-124.

Warren, A.J. (2018). Molecular basis of the human ribosomopathy Shwachman-Diamond syndrome. Advances in biological regulation 67, 109-127.

Weis, F., Giudice, E., Churcher, M., Jin, L., Hilcenko, C., Wong, C.C., Traynor, D., Kay, R.R., and Warren, A.J. (2015). Mechanism of eIF6 release from the nascent 60 S ribosomal subunit. Nat Struct Mol Biol 22, 914-919.

Wilson, A., Laurenti, E., Oser, G., van der Wath, R.C., Blanco-Bose, W., Jaworski, M., Offner, S., Dunant, C.F., Eshkind, L., Bockamp, E., et al. (2008). Hematopoietic stem cells reversibly switch from dormancy to self-renewal during homeostasis and repair. Cell 135, 1118-1129.

Woloszynek, J.R., Rothbaum, R.J., Rawls, A.S., Minx, P.J., Wilson, R.K., Mason, P.J., Bessler, M., and Link, D.C. (2004). Mutations of the SBDS gene are present in most patients with Shwachman-Diamond syndrome. Blood 104, 3588-3590.

Wong, C.C., Traynor, D., Basse, N., Kay, R.R., and Warren, A.J. (2011). Defective ribosome assembly in Shwachman-Diamond syndrome. Blood 118, 4305-4312.

Zohn, I.E., Anderson, K.V., and Niswander, L. (2007). The Hectd1 ubiquitin ligase is required for development of the head mesenchyme and neural tube closure. Dev Biol 306, 208-221. 
Figure 1. Lv, et al
A

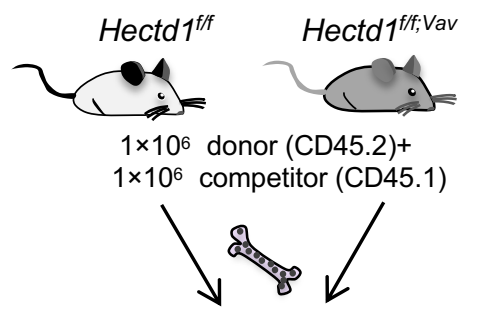

Primary BMT

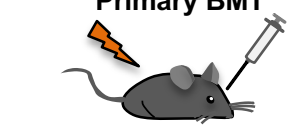

CD45.1/2

$1 \times 10^{6}$ BMs Secondary BMT

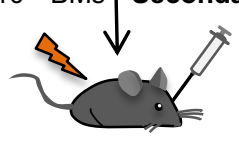

CD45.1/2

E

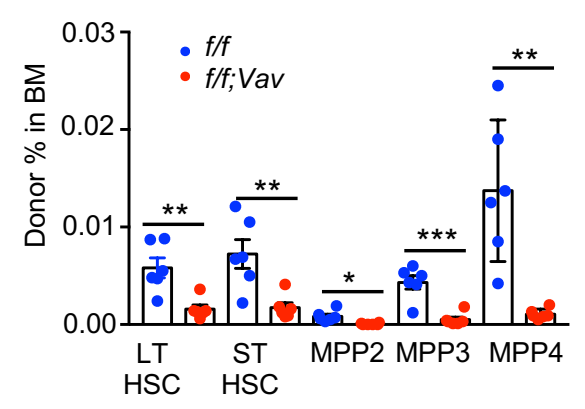

G

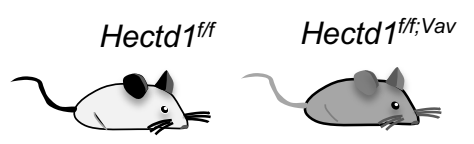

Various donor doses (CD45.2)+ $3 \times 10^{5}$ competitor (CD45.1)

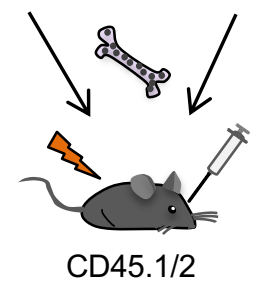

H
B
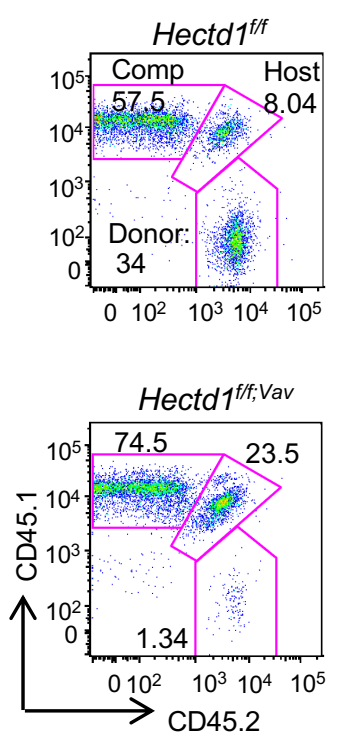

C

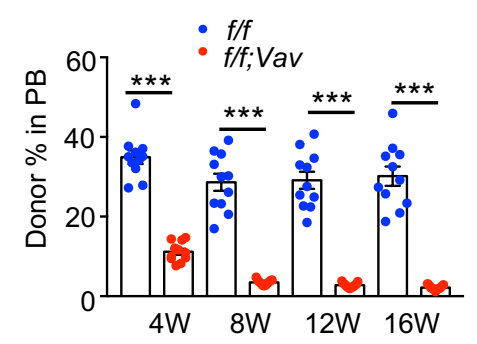

D

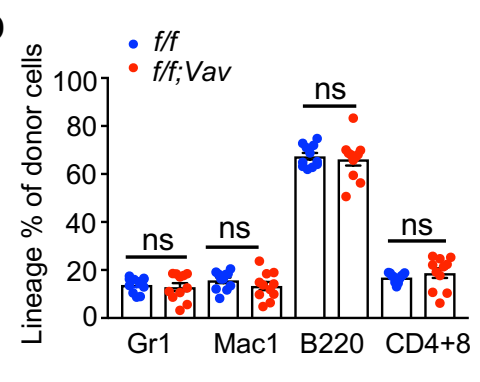

F Secondary BMT

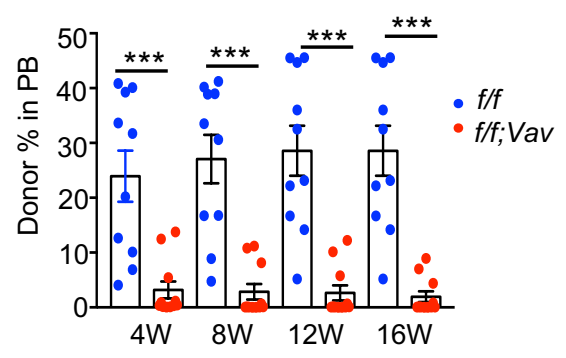

\begin{tabular}{|l|l|l|}
\hline Dose & Hectd 1ff & Hectd 1ff; Vav \\
\hline 10,000 & $5 / 9$ & $1 / 7$ \\
\hline 30,000 & $9 / 10$ & $4 / 13$ \\
\hline 100,000 & $5 / 5$ & $6 / 6$ \\
\hline CRU & 1 in $\mathbf{1 3 , 2 0 0}$ & $\mathbf{1}$ in $\mathbf{5 5 , 3 0 0}$ \\
\hline $\begin{array}{l}\text { Range } \\
\pm 1 S E\end{array}$ & $\begin{array}{l}1 \text { in } 23,700 \text { to } \\
1 \text { in } 7,460\end{array}$ & $\begin{array}{l}1 \text { in } 101,300 \text { to } \\
1 \text { in } 30,310\end{array}$ \\
\hline $\begin{array}{l}\text { 2-tailed } \\
\text { t-test }\end{array}$ & \multicolumn{2}{|c|}{ p<0.001 } \\
\hline
\end{tabular}

I

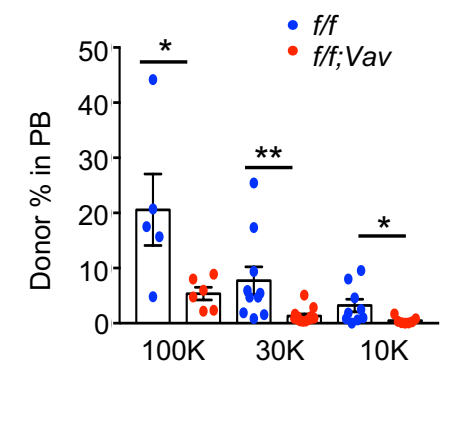


Figure 2. Lv, et al (smaller size)

A

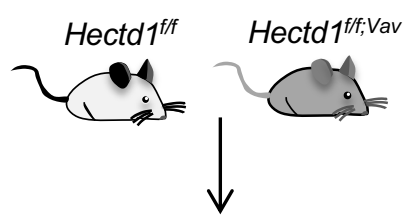

LT-HSC (LSK CD150+CD48-)

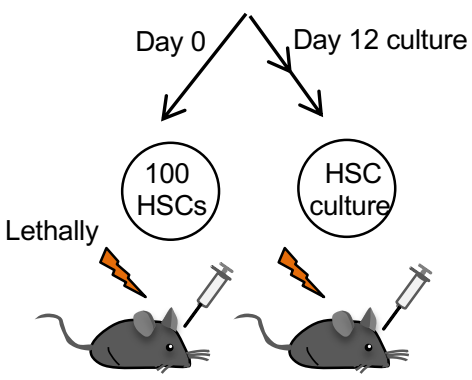

$\mathbf{F}$

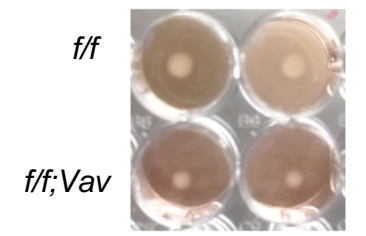

J

Hectd $1^{\text {fff }} \quad$ Hectd $1^{\text {fff; }}$ Vav

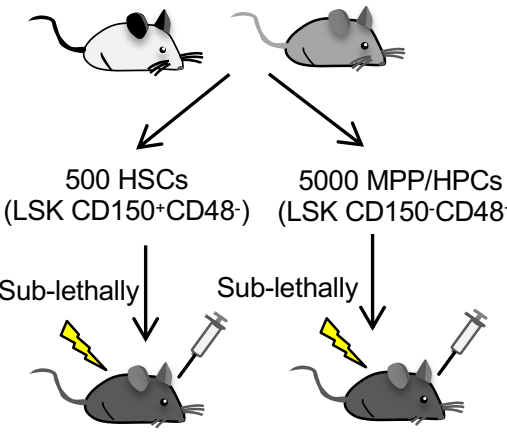

Check donor chimerism every week post-BMT
B

Fresh HSC (Day 0)

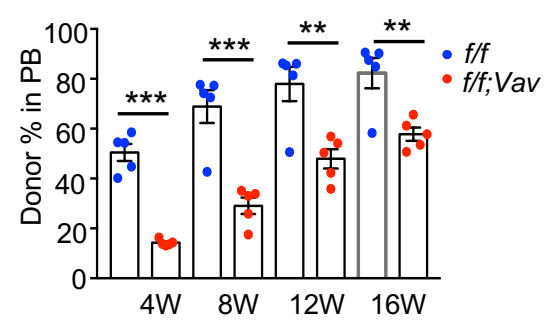

D

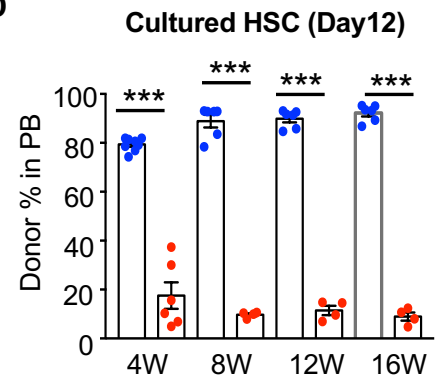

C

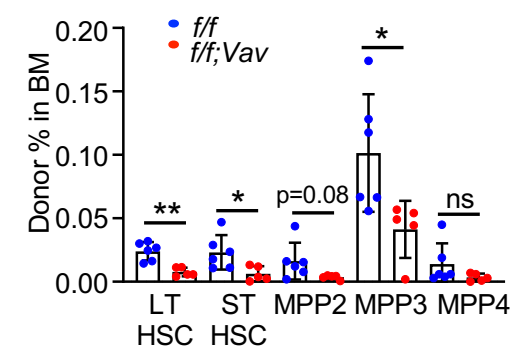

E

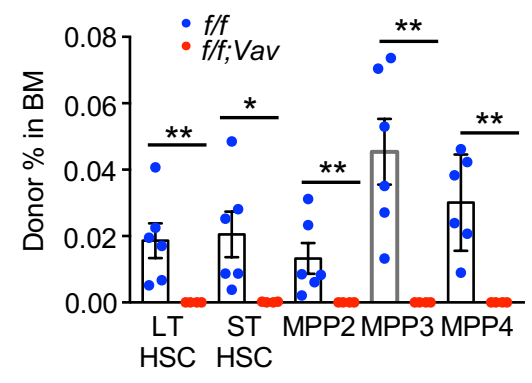

G

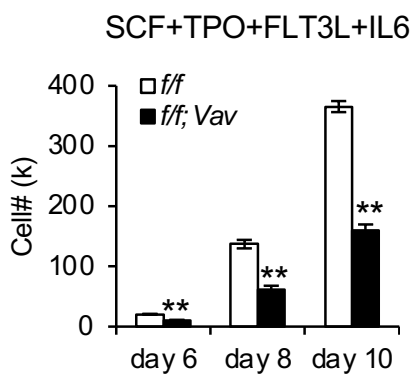

H

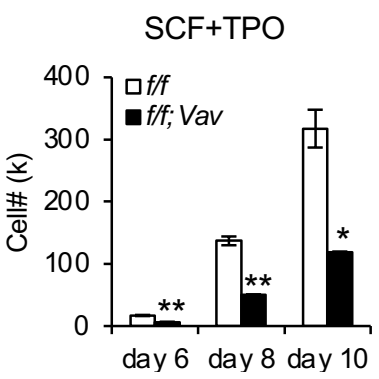

K

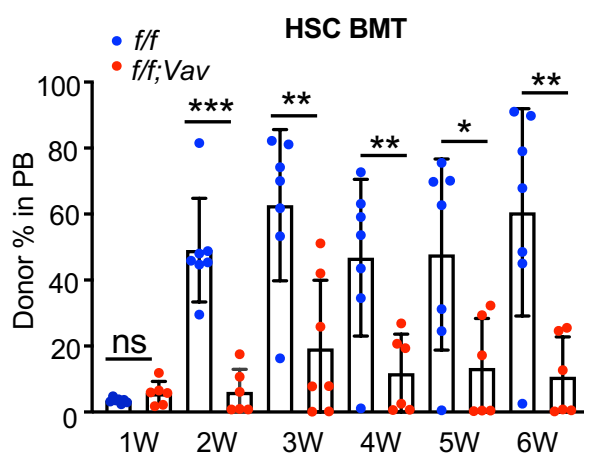

$\mathbf{L}$

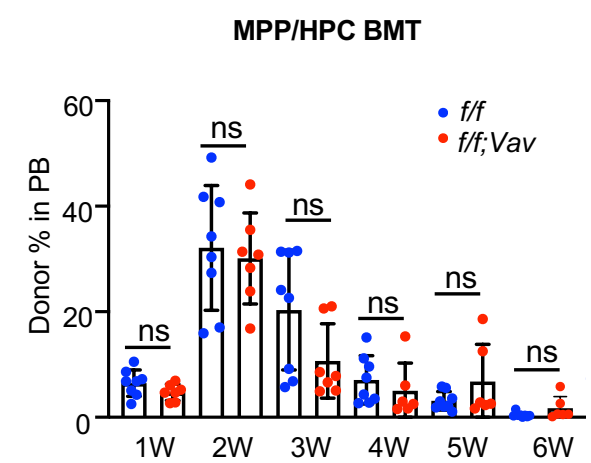


Figure 3. Lv, et al (smaller size)

A

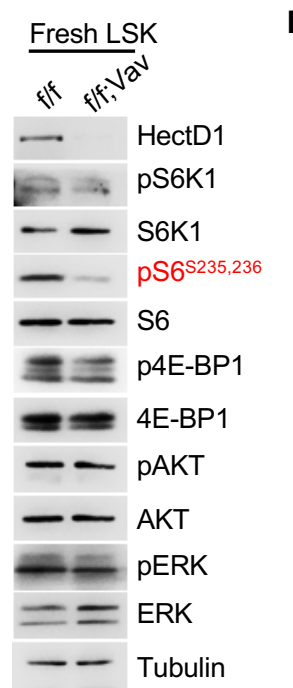

B

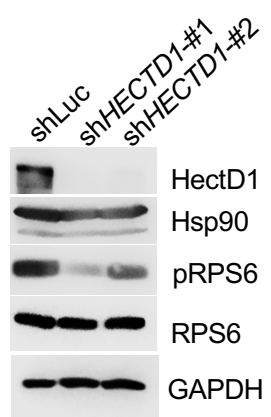

E

HA HA Glycine Glycine Beads Elute1 Elute2 Elute1 Elute2 boil

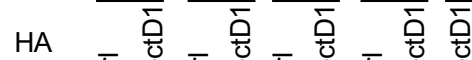

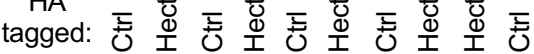

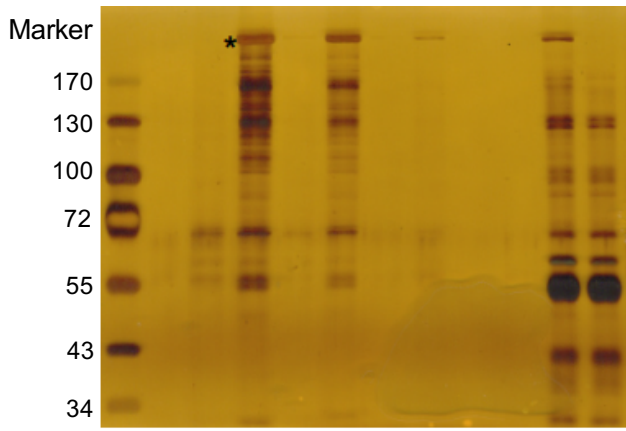

HectD1

$\lg G-H$

G

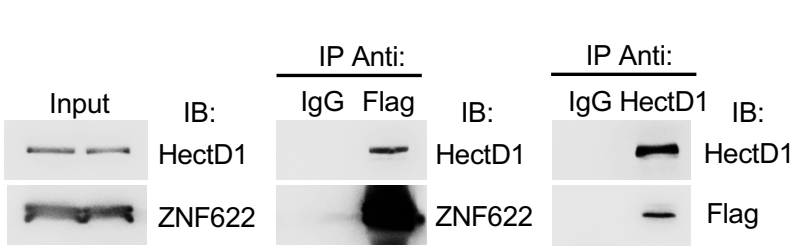

M
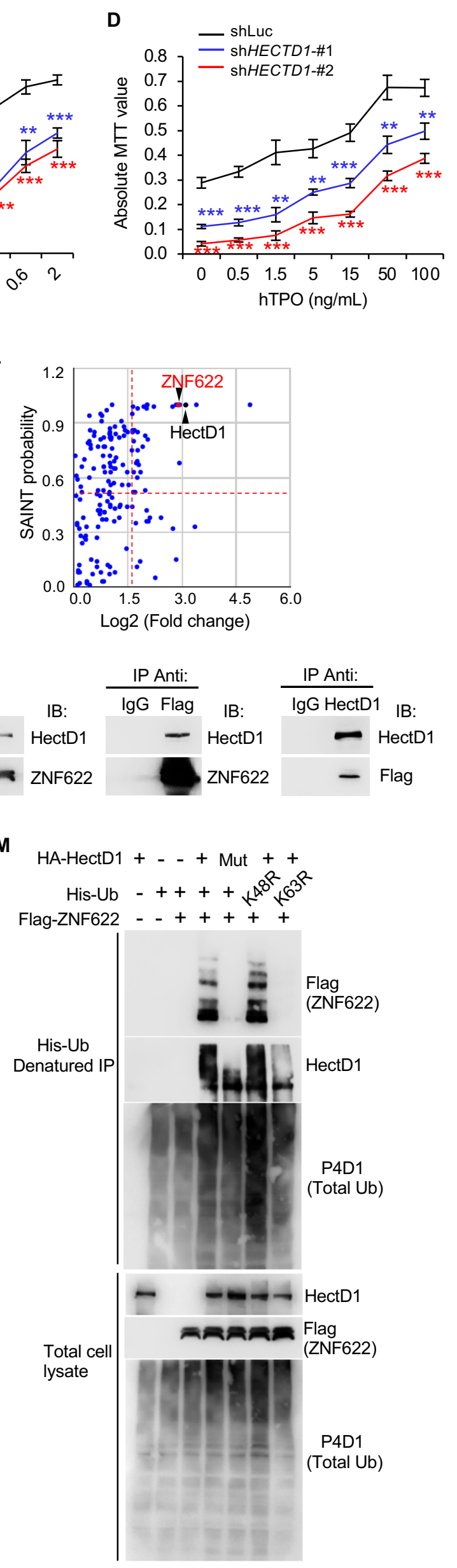

$\mathbf{F}$

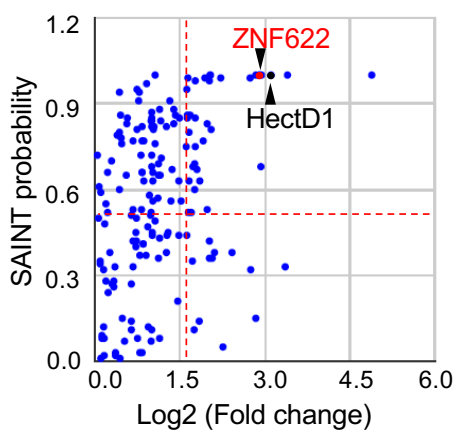

H

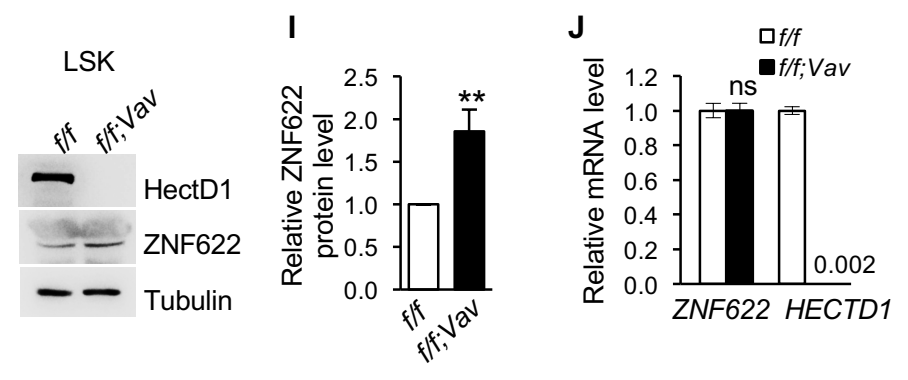

K

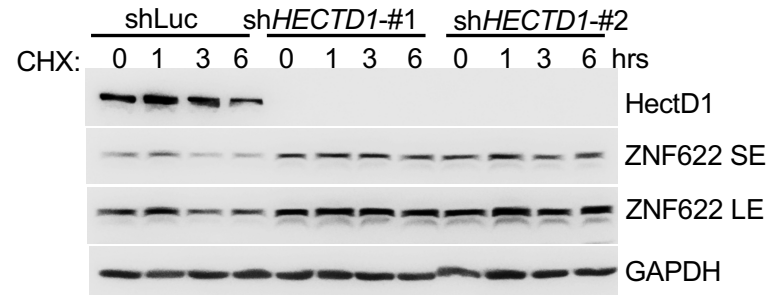

$\mathbf{L}$
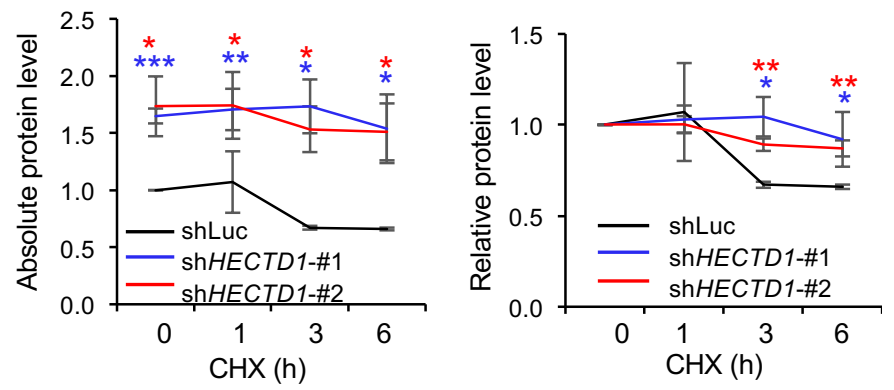
Figure 4. Lv, et al

A

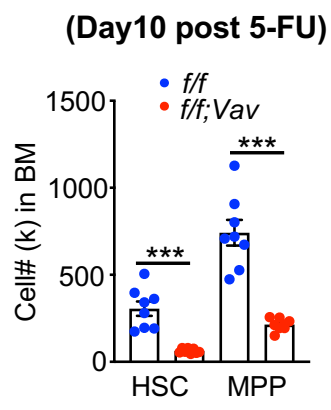

B

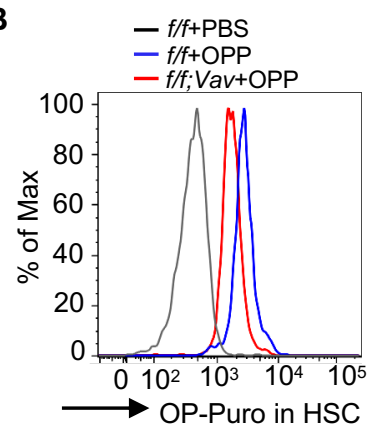

E

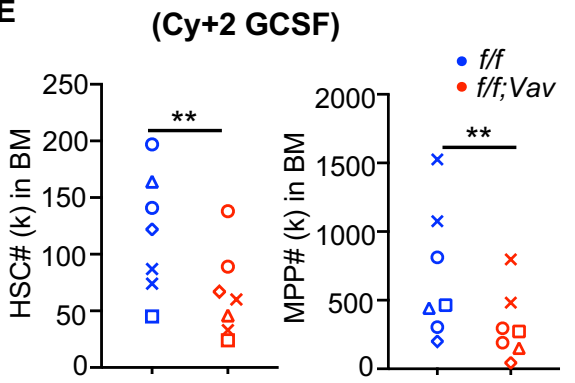

$\mathbf{2} \quad f / f+\mathrm{PBS}$
- $f / f+\mathrm{OPP}$

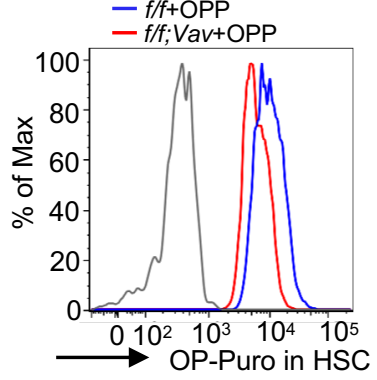

C

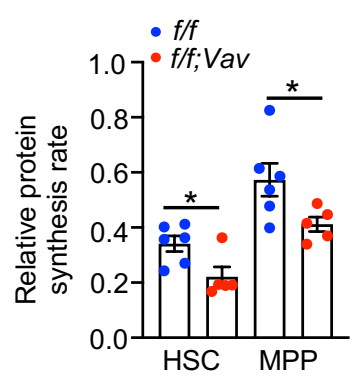

G

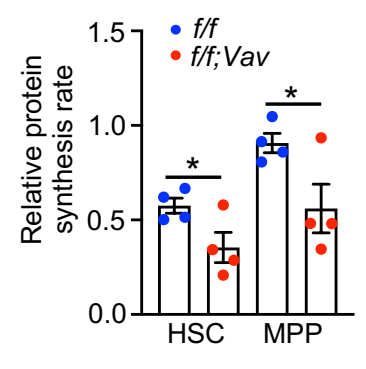

D

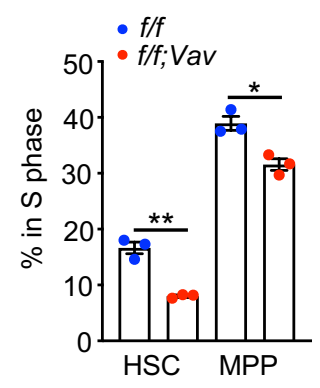

H

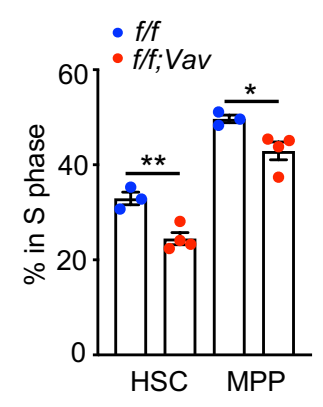

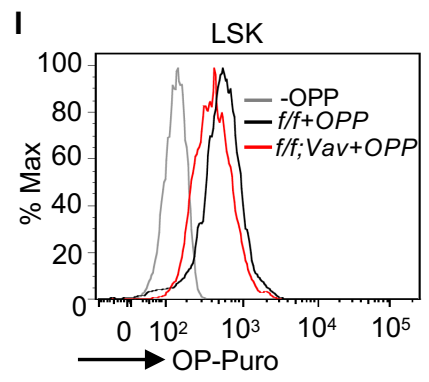
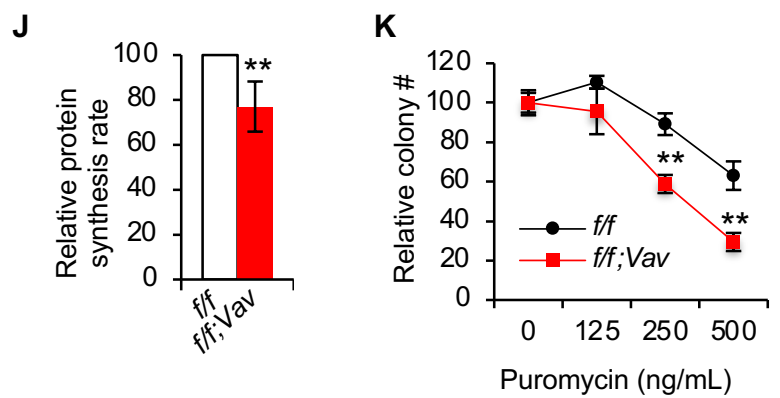


\section{Figure 5. Lv, et al}
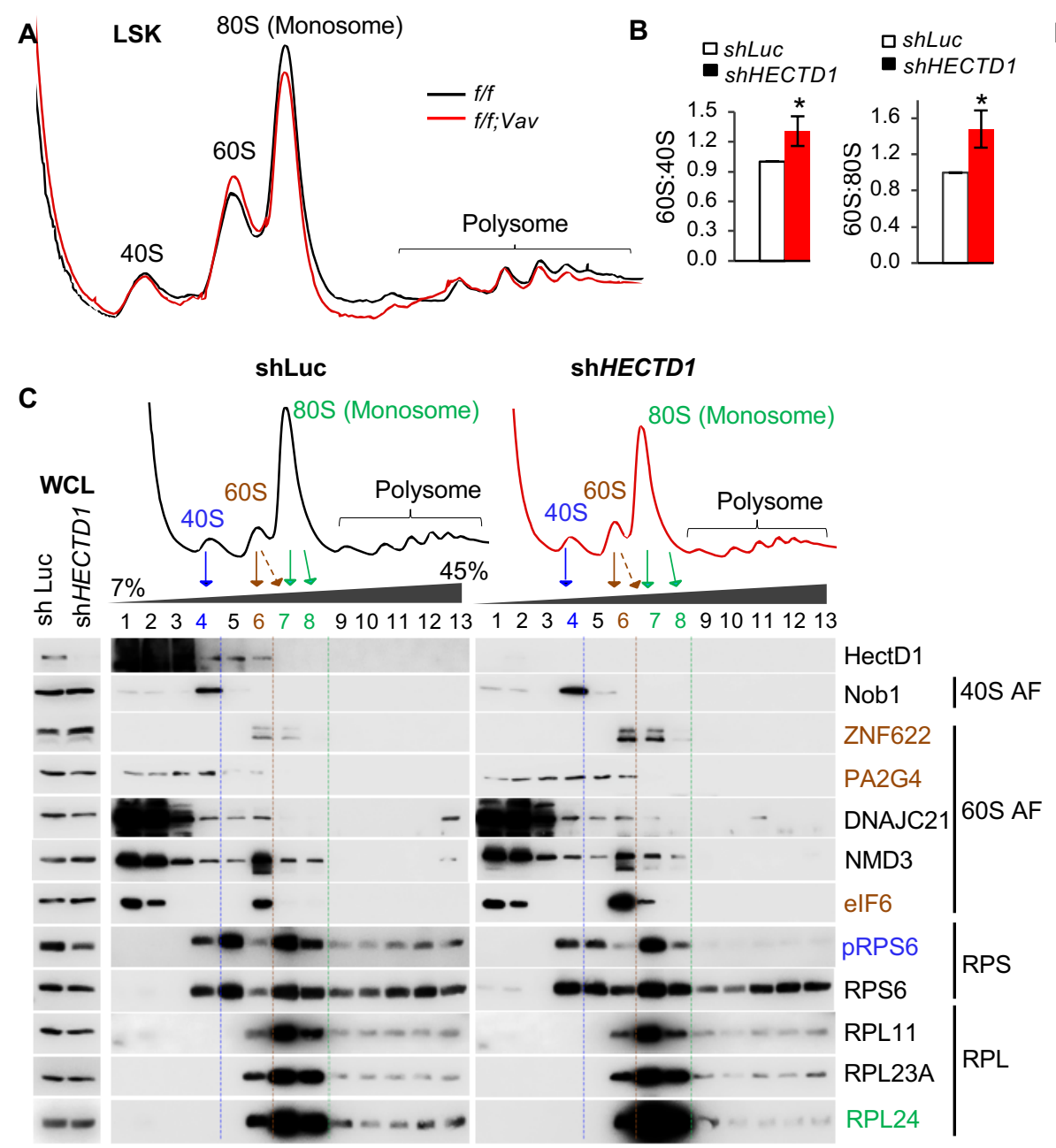

E

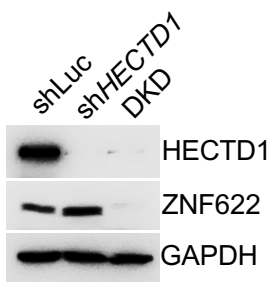

$\mathbf{F}$

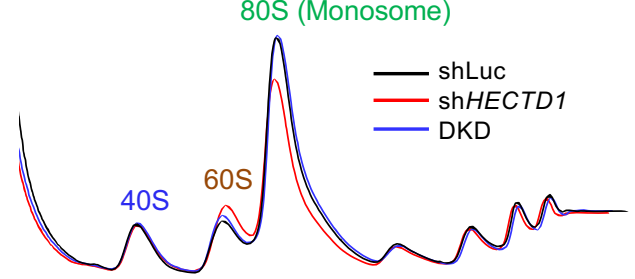

D

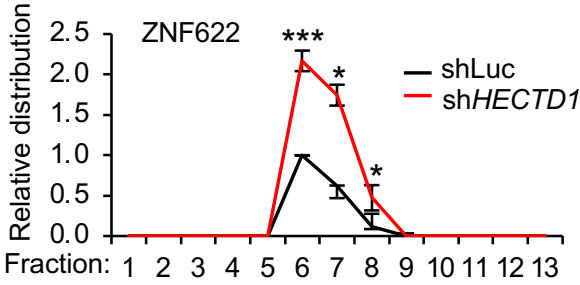

Fraction: $1 \begin{array}{llllllllll}2 & 3 & 4 & 5 & 6 & 7 & 8 & 9 & 1011 & 1213\end{array}$

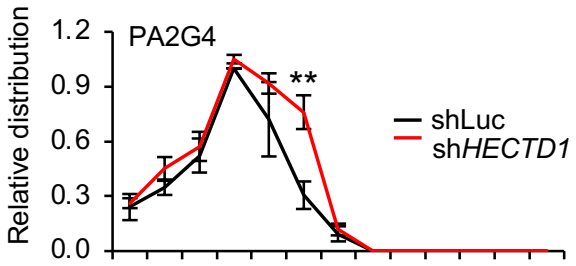

Fraction:1 $2 \begin{array}{lllllllll}3 & 4 & 5 & 6 & 7 & 8 & 9 & 1011 & 1213\end{array}$

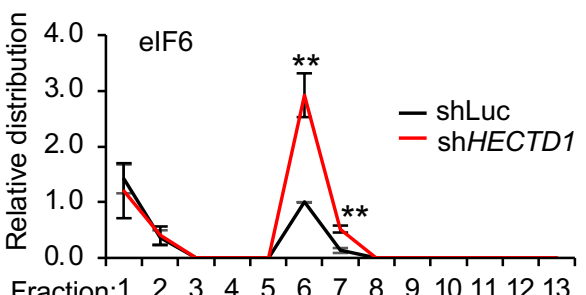

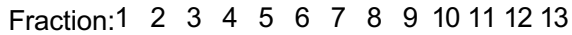

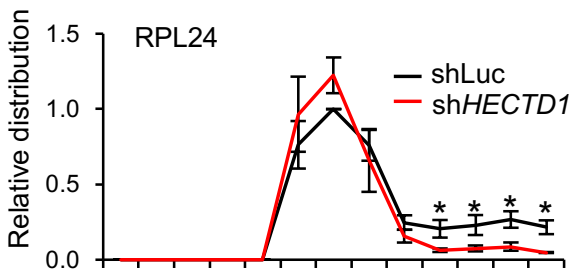

Fraction: $1 \quad 2 \quad 3 \quad 4 \quad 5 \quad 6 \quad 7 \quad 8 \quad 910111213$
H

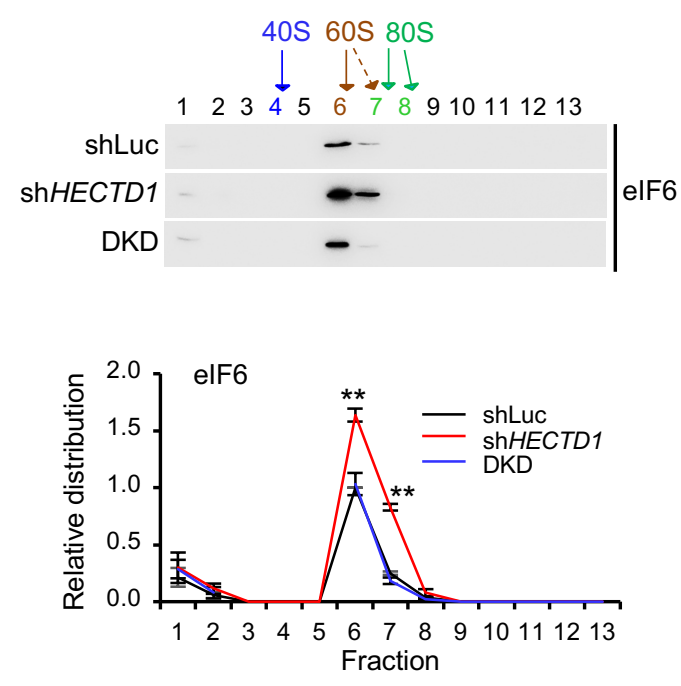

\section{I}

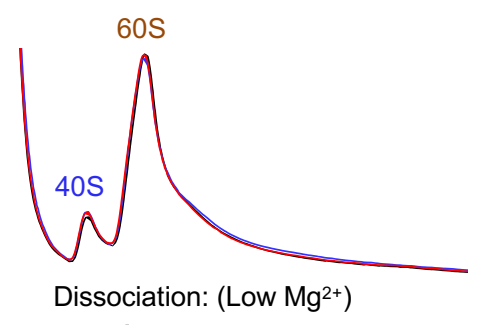

$\downarrow$ Add $\mathrm{Mg}^{2+}$

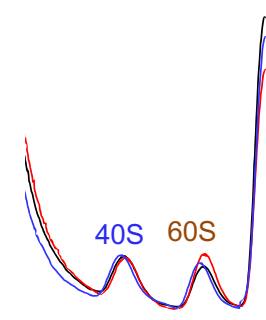

G

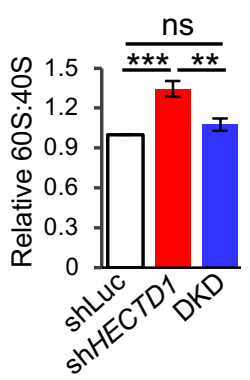

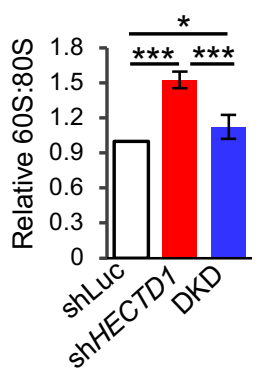

J
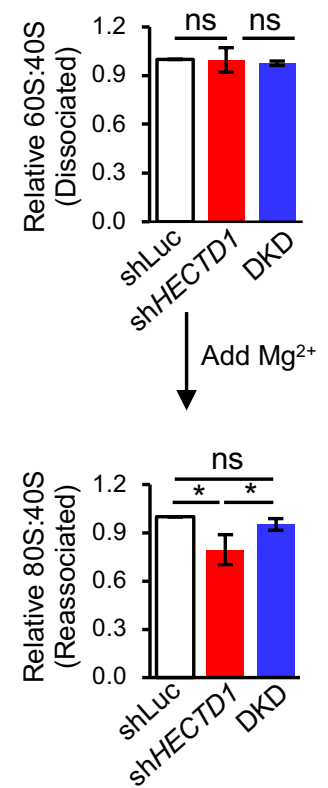
Figure 6. Lv, et al
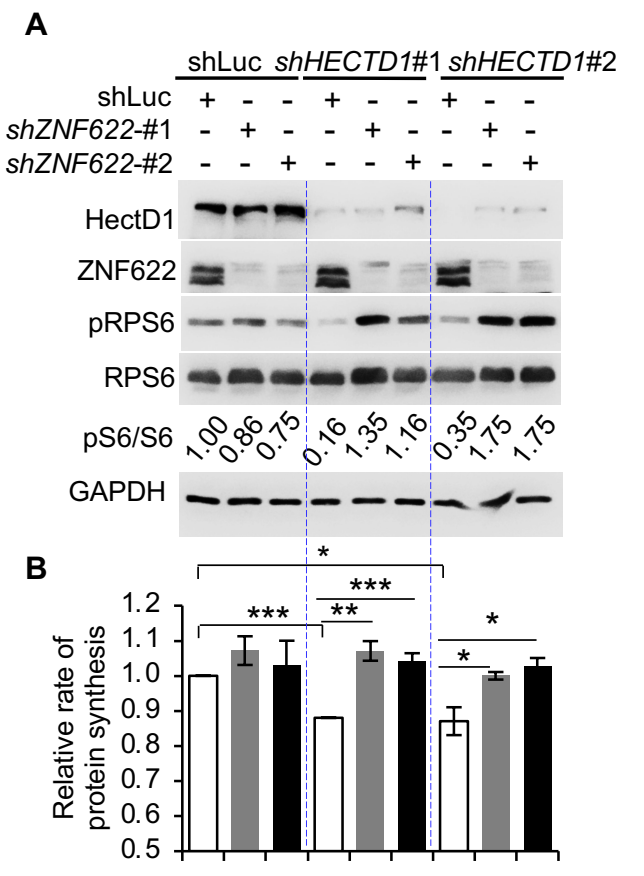

G

O $f / f+s h L u c$

- $f / f+s h Z n f 622 \# 1$

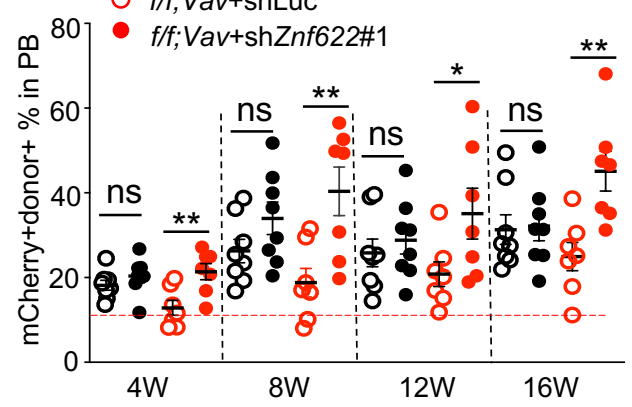

C

$\stackrel{\circlearrowright}{\frac{0}{\omega}} \frac{s h m Z N F 622}{\# 1 \# 2 \# 3}$

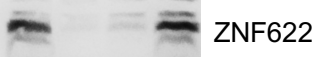

$-\infty$ GAPDH
Seseses Hsp90

D

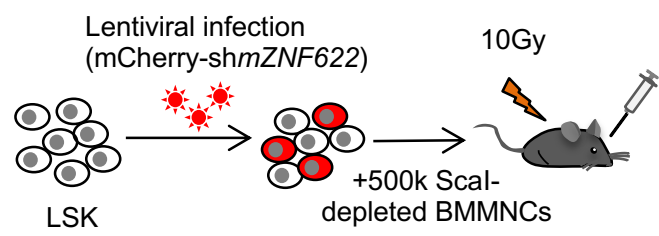

E

- $f f f: V a v+s h L u c$

- f/f;Vav+shZnf622\#1

- f/f;Vav+shZnf622\#2

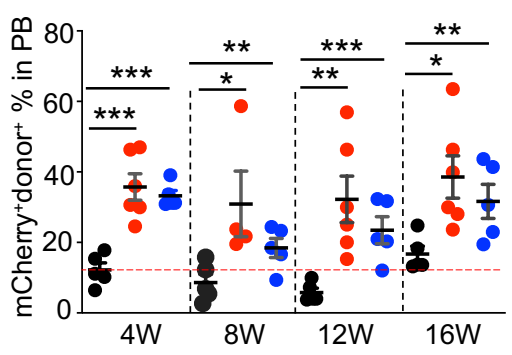

F

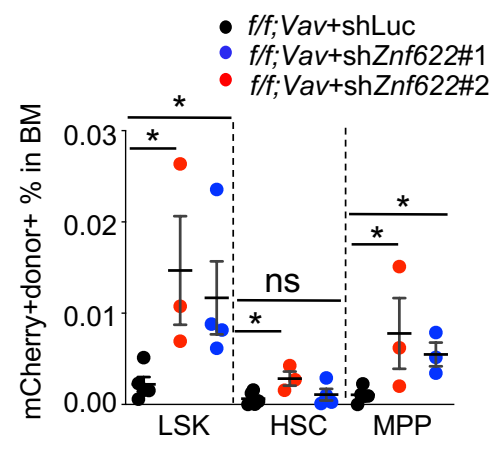

H

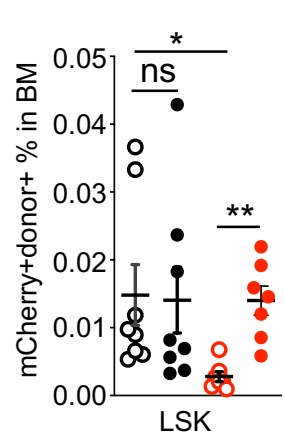

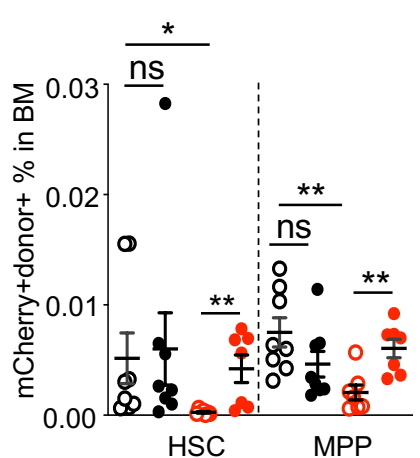

I

Secondary BMT

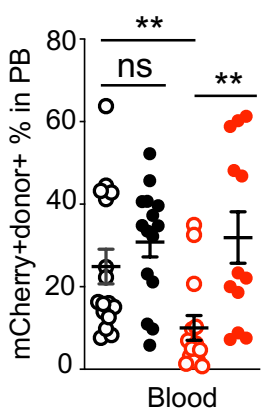


A

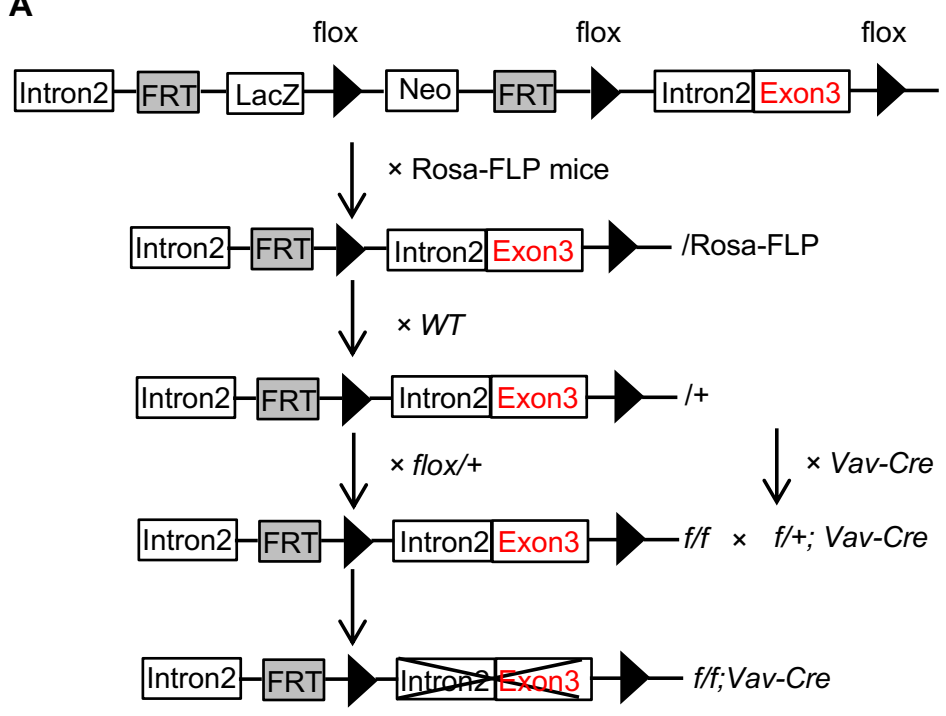

Exon 3 excision results in a 50 AA HectD1 truncated protein.
B

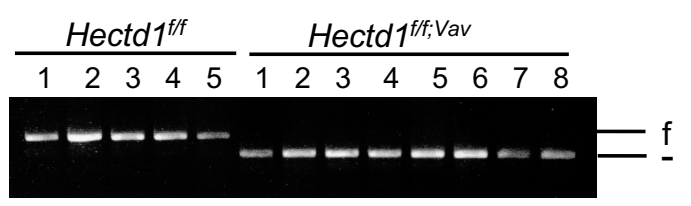

C

Exon3 Primer pair 1
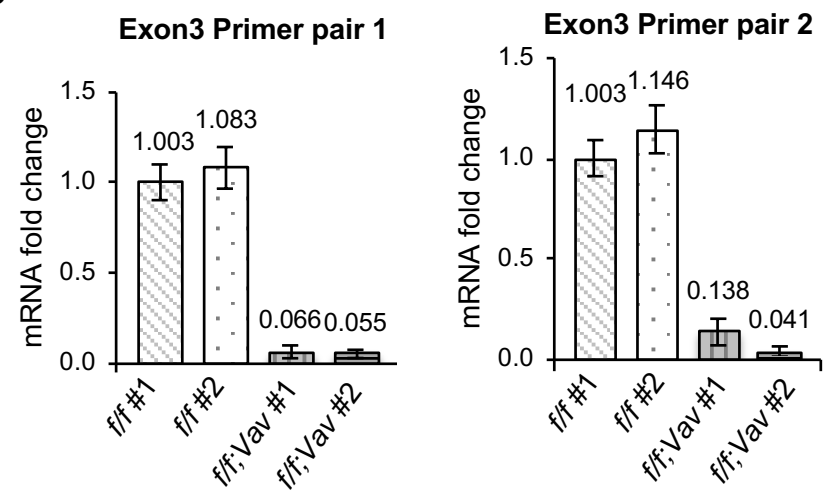

E

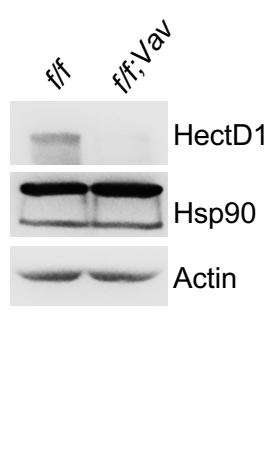

Figure S1. Generation of hematopoietic-specific Hectd1 conditional knockout mice. Related to Figure 1.

(A) Strategy of generating Vav-cre mediated conditional Hectdl knockout (Hectd ${ }^{\text {ff; }}{ }^{\text {Vav }}$ ) mice. Exon3 was flanked by floxP sites. Deletion of exon 3 results in an early stop codon in exon4, producing a 50 aa (amino acid) truncated protein.

(B) Genotyping of individual bone marrow-derived hematopoietic colonies from Hectd ${ }^{\mathrm{fff}}$ and Hectd $1^{\mathrm{ff} \text {; Vav }}$ mice to evaluate Hectdl deletion efficiency.

(C) RT-qPCR analysis to evaluate the deletion efficiency of Hectdl at the mRNA level. Hectdl contains 43 exons. qPCR primer pairs targeting to exon 3 are designed and used for qPCR analysis. BM cells from two mice of each genotype were tested.

(D) As negative controls, qPCR primer pairs targeted to Hectdl exon 6, 22, 38 were designed and used to confirm the specificity of Hectdl excision in mice.

(E) Western blot analysis of BM cells to confirm Hectdl knockout efficiency at the protein level. Data are represented by mean $\pm \mathrm{SD}$ in $(\mathrm{C}, \mathrm{D})$. 
A

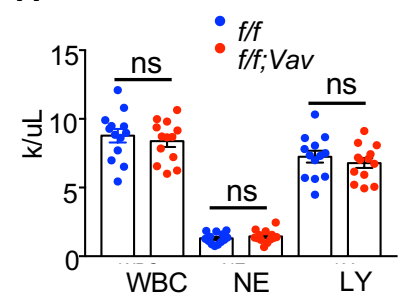

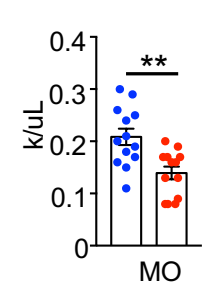

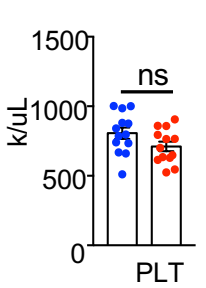

E

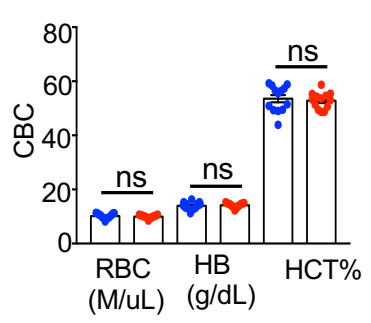

B
C

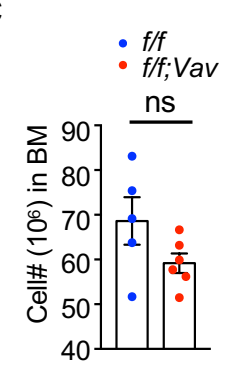

D $\quad: f / f f$ fff; $\operatorname{Vav}$

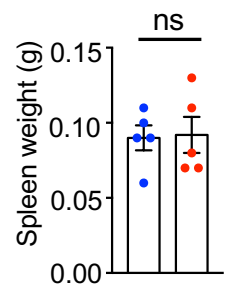

$0.20] \cdot f / f$

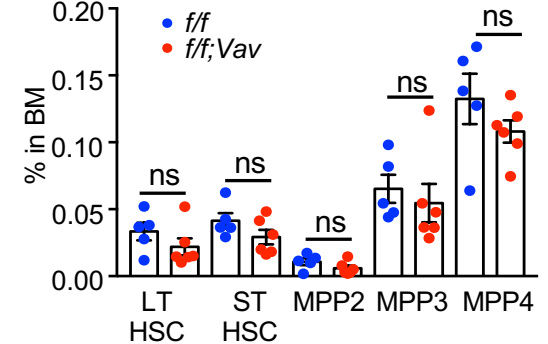

$\mathbf{F}$

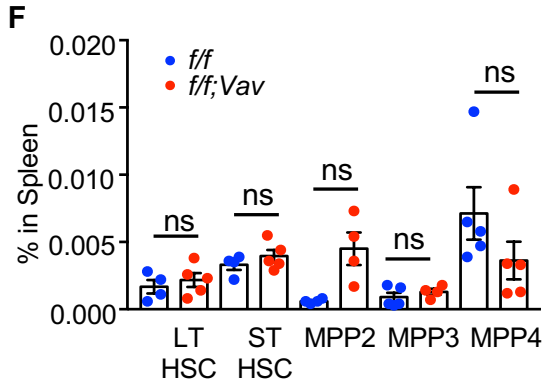

G

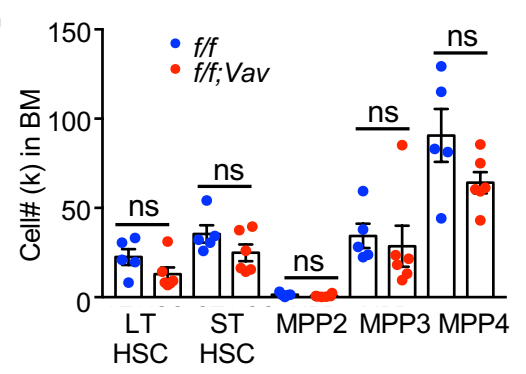

H

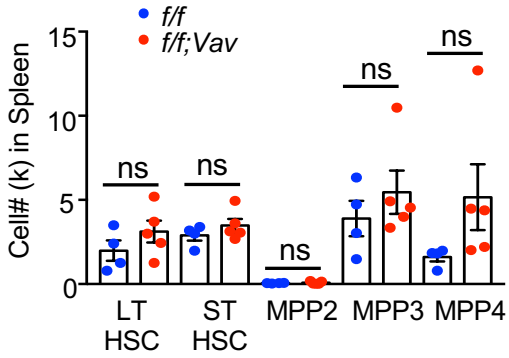

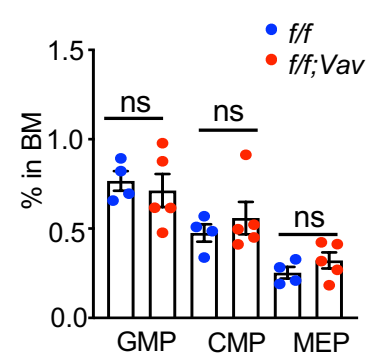

L

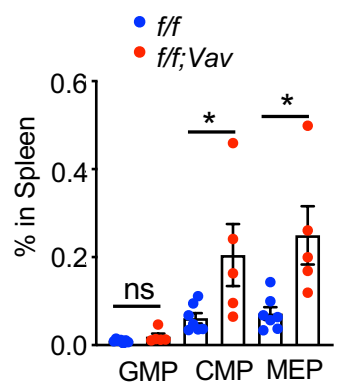

0

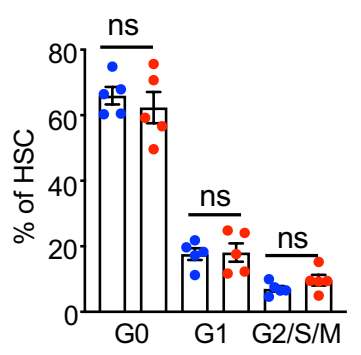

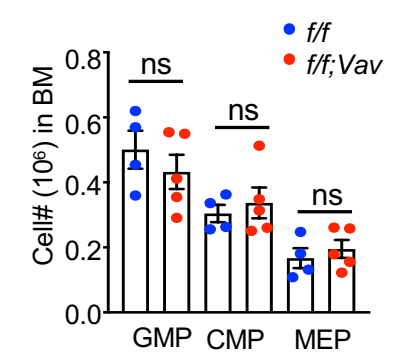

M

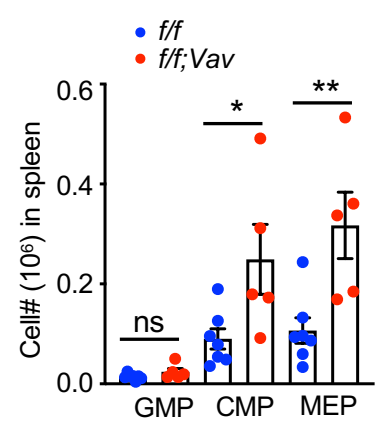

K

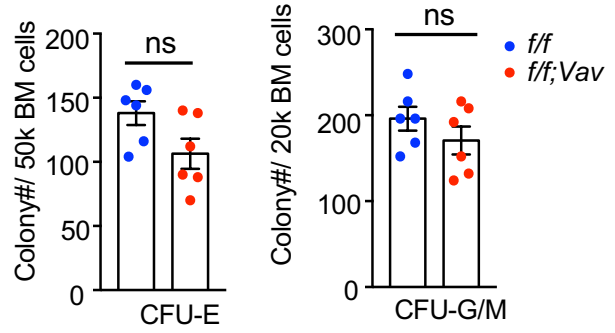

$\mathbf{N}$
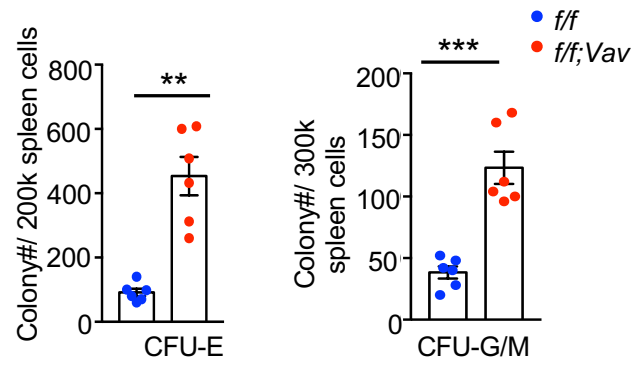
Figure S2. Hematopoietic-specific Hectd1 knockout mice show relatively normal steady-state hematopoiesis. Related to Figure 1.

(A) Complete blood count (CBC) analysis of peripheral blood (PB) from Hectd1 ${ }^{\text {fff }}\left(\mathrm{n}=13\right.$ ) and Hectd1 ${ }^{\text {ff; Vav }}$ ( $\mathrm{n}=13$ ) mice. WBC, whole blood count; NE, neutrophils; LY, lymphocytes; MO, monocytes; PLT, platelets; RBC, red blood cells; HB, hemoglobin; HCT, hematocrit.

(B) Lineage cell percentages in the PB of Hectd $1^{f f f}(\mathrm{n}=8)$ and Hectd $1^{\text {ff; } V a v}(\mathrm{n}=11)$ mice were analyzed by flow cytometry.

(C) Total BM cellularity of Hectdl $I^{f / f}(\mathrm{n}=5)$ and Hectdl $1^{\text {ff; }: \text { Vav }}(\mathrm{n}=6)$ mice.

(D) Spleen weight of Hectd ${ }^{f f f}(\mathrm{n}=5)$ and Hectdl ${ }^{\text {ff; }: \operatorname{Vav}}(\mathrm{n}=5)$ mice.

$(\mathrm{E}, \mathrm{F})$ Flow cytometry analysis of the frequency of various HSPC subpopulations in BM (E) and spleen (F) of Hectd $1^{f f f}$ $(\mathrm{n}=5)$ and Hectd $1^{f f ; \text { Vav }}(\mathrm{n}=6)$ mice. Long-term hematopoietic stem cell (LT-HSC) is defined as CD150 CD48-F1k2-LSK cells. Short-term HSC (ST-HSC): CD150CD48-Flk2-LSK. MPP2: CD150 CD48 ${ }^{+}$Flk2-LSK. MPP3: CD150-CD48 ${ }^{-}$Flk2 ${ }^{-}$ LSK. MPP4: CD150- Flk2 ${ }^{+}$LSK.

$(\mathrm{G}-\mathrm{H})$ Cell numbers of HSPC subpopulations in BM $(\mathrm{G})$ and spleen $(\mathrm{H})$ of Hectd $1^{\mathrm{fff}}(\mathrm{n}=5)$ and Hectd1 ${ }^{\text {ff;:Vav }}(\mathrm{n}=5)$ mice.

(I, J) Frequency (I) and cell number (J) of different hematopoietic progenitors in BM of Hectdlfff $(\mathrm{n}=5)$ and Hectdl ff;:Vav $(n=6)$ mice as determined by flow cytometry are shown.

(K) Colony-forming units (CFU)-E (left) and CFU-G/M (right) of the BM are shown.

$(\mathrm{L}, \mathrm{M})$, Frequency $(\mathrm{L})$ and cell number $(\mathrm{M})$ of different hematopoietic progenitors in the spleen of Hectd $1^{\mathrm{fff}}(\mathrm{n}=5)$ and Hectd $1^{\text {ff; } \text { :Vav }}(\mathrm{n}=6)$ mice are shown.

(N) CFU-E (left) and CFU-G/M (right) analysis of the spleen are shown.

(O-P) Cell cycle analysis of HSCs (O) and MPPs $(\mathrm{P})$ in primary Hectd $1^{f f f}(\mathrm{n}=5)$ and Hectdl ${ }^{\mathrm{f} f ; \text { Vav }}(\mathrm{n}=5)$ mice as determined by Ki67 and DAPI.

(Q) Cell apoptosis analysis of LSKs, HSCs and MPPs in primary Hectdl $1^{f f f}(\mathrm{n}=4)$ and Hectd1 $1^{\text {ff;:Vav }}(\mathrm{n}=3)$ mice by Annexin V staining.

All the experiments above were performed in 8-10 weeks old young mice. Each symbol represents an individual mouse; bars indicate mean values; error bars indicate SE. *: $\mathrm{p}<0.05$; **: $\mathrm{p}<0.01$; ***: $\mathrm{p}<0.001$; ns: not significant, as determined by two-tailed Student's $t$-tests. 
A

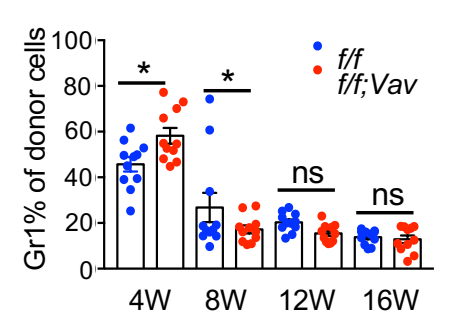

E

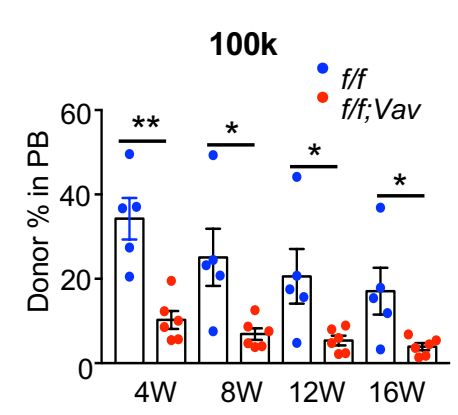

H

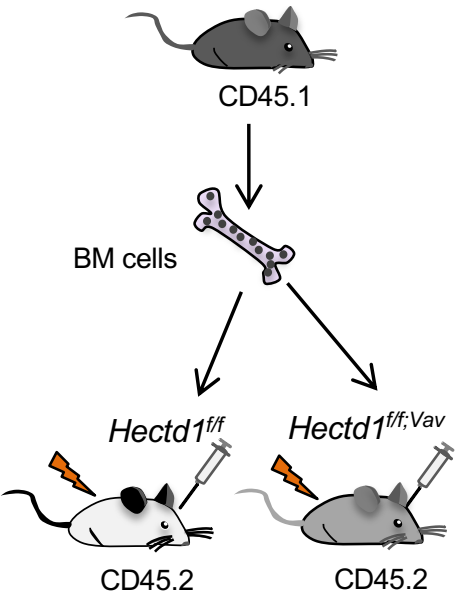

L

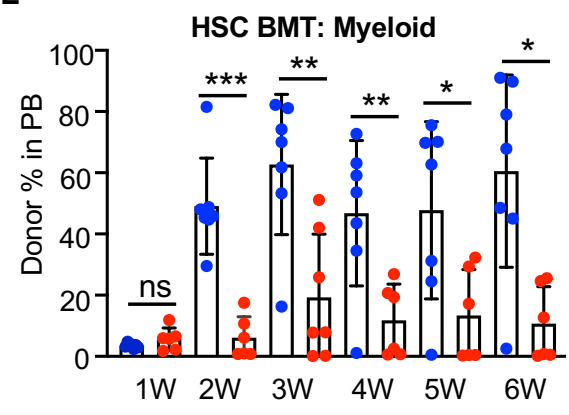

M

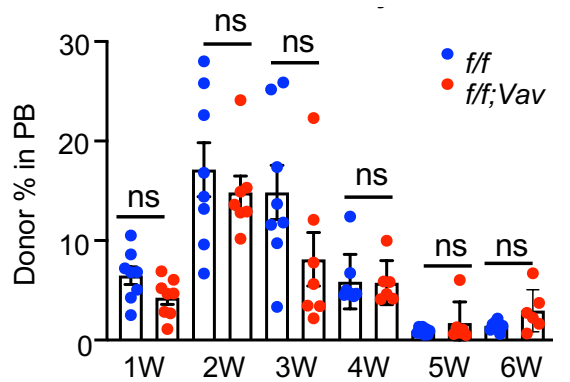

C

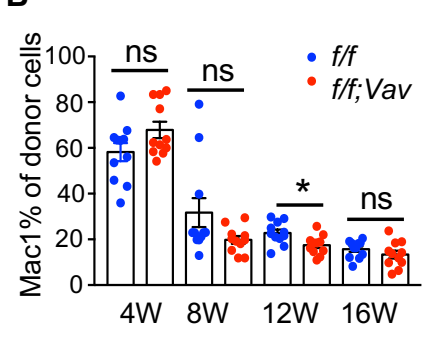

D

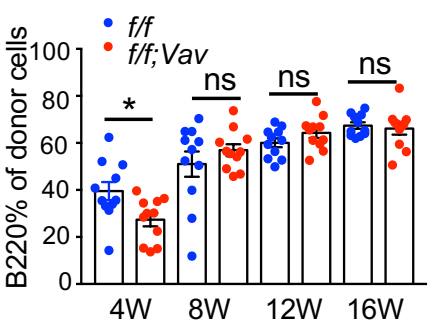

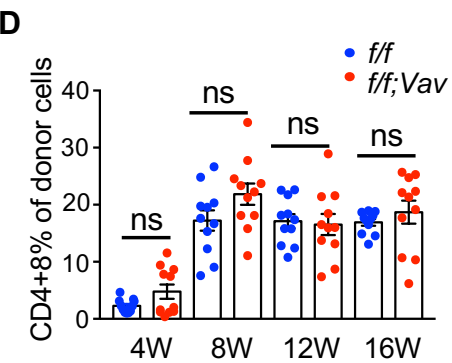

F

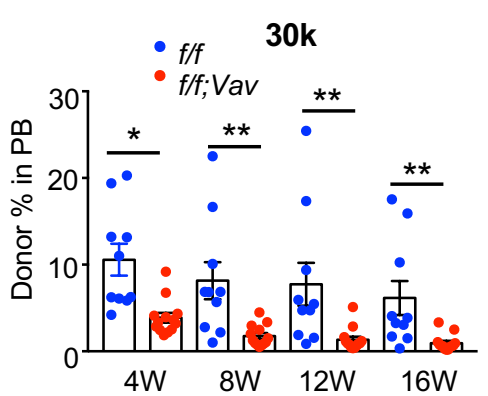

G

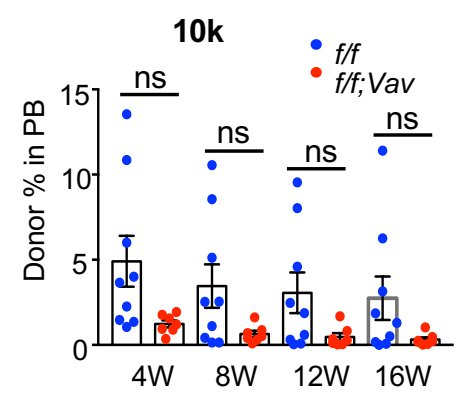

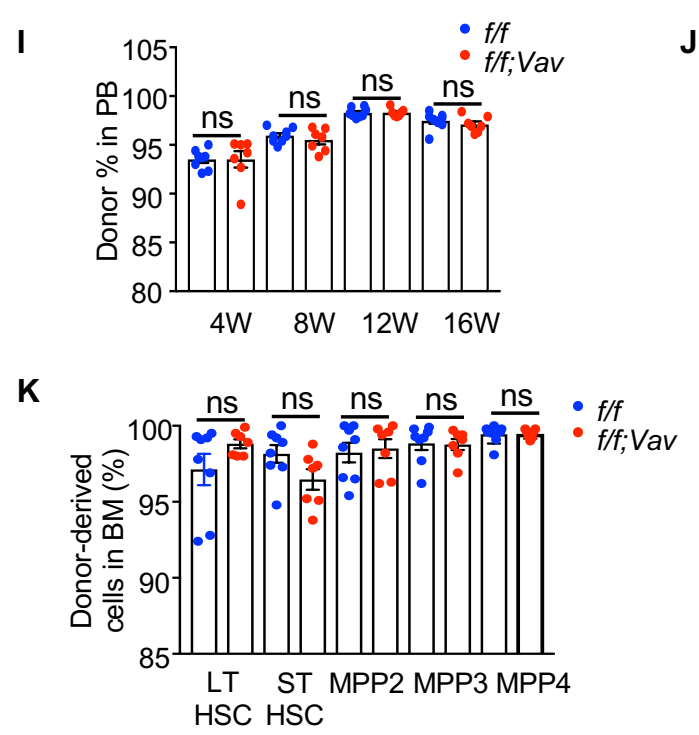
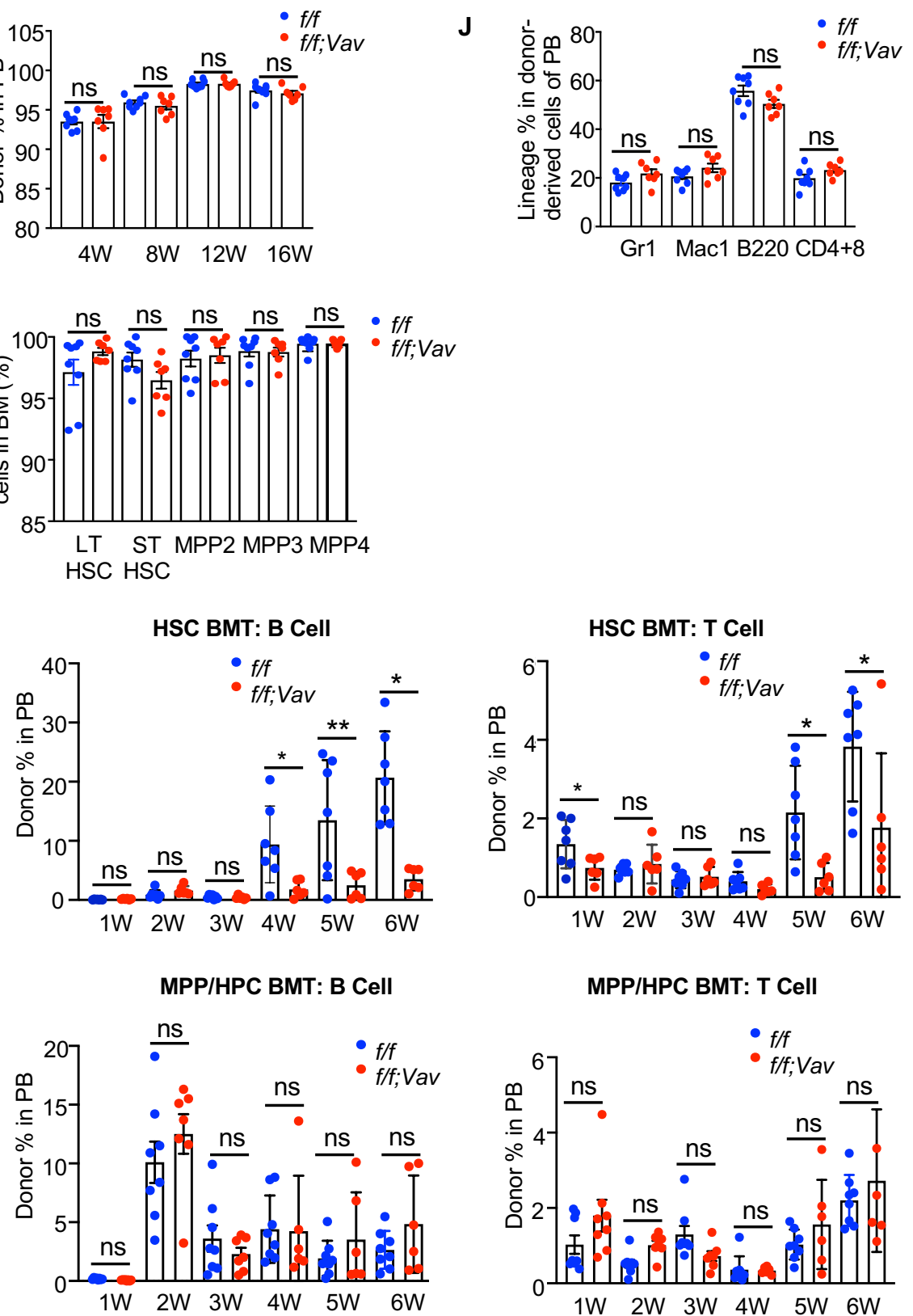

MPP/HPC BMT: T Cell

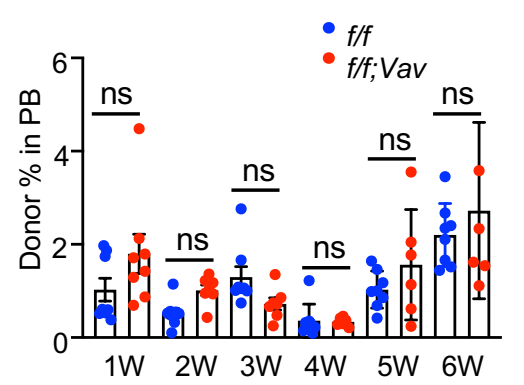


Figure S3. Loss of Hectd1 does not impair hematopoietic differentiation, bone marrow niche or MPP reconstitution in transplanted mice. Panels S3A-S3K are related to figure 1 and panels S3L-S3M are related to figure 2.

(A-D) Loss of Hectd1 does not impair hematopoietic differentiation in transplanted mice. Examination of donor HSC differentiation in competitive total BM transplanted mice. $1^{*} 10^{6}$ unfractionated BM cells from Hectd $I^{f f f}$ and Hectd $I^{f f ; \text { Vav }}$ mice were injected with the same number of competitor BM cells into lethally irradiated recipient mice. Donor chimerisms in different lineages of the PB were shown. (A) Granulocytes, (B) monocytes, (C) B cells and (D) T cells. Hectd $1^{f / f}(\mathrm{n}=11)$ and Hectd ${ }^{f f f ; V a v}(\mathrm{n}=11)$.

(E-G) Hectd1 deficiency leads to a decreased donor reconstitution in limiting dilution BMT. Donor cell percentage in recipients' $\mathrm{PB}$ injected with $100 \mathrm{k}(\mathrm{E}), 30 \mathrm{k}(\mathrm{F}), 10 \mathrm{k}(\mathrm{G})$ donor BM cells mixed with $300 \mathrm{k}$ competitor BM cells were examined

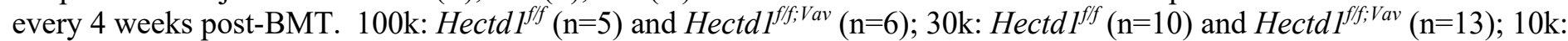
$\operatorname{Hectd}^{\mathrm{fff}}(\mathrm{n}=9)$ and Hectd $I^{\mathrm{ff} ; \mathrm{Vav}}(\mathrm{n}=7)$.

(H-K) Hectd1 deficiency does not affect bone marrow niche as shown in reciprocal BMT.

(H) Experimental scheme of reciprocal BMT. $1 \times 10^{6}$ total BM cells from SJL mice (CD45.1) were transplanted into lethallyirradiated Hectd $1^{f f f}(\mathrm{n}=8)$ and Hectd $1^{\text {ff;:Vav }}(\mathrm{n}=7)$ littermates (CD45.2).

(I) Donor chimerism was analyzed by flow cytometry every 4 weeks post-BMT.

(J) Donor-derived cells in each lineage 16 weeks post-BMT are shown.

(K) Donor percentages in different HSPC subpopulations in the BM of Hectdl fff and Hectd $1^{f f ; \text { Vav }}$ recipient mice are shown. (L-M) HectD1 is required for HSC but not MPP/HPC reconstitution ability.

(L) Percentages of donor HSC-derived myeloid cells $\left(\mathrm{Gr} 1 / \mathrm{Mac1}^{+}\right)$, B cells $\left(\mathrm{B} 220^{+}\right)$and $\mathrm{T}$ cells $\left(\mathrm{CD} 4^{+} \mathrm{CD} 8^{+}\right)$are shown.

(M) Percentages of donor MPP/HPC-derived myeloid cells $\left(\mathrm{Gr} 1 / \mathrm{Mac1}^{+}\right)$, B cells $\left(\mathrm{B} 220^{+}\right)$and $\mathrm{T}$ cells $\left(\mathrm{CD} 4^{+} \mathrm{CD} 8^{+}\right)$are shown.

In all relevant panels, each symbol represents an individual mouse; bars indicate mean frequencies; error bars indicate SE. *: $\mathrm{p}<0.05 ; * *: \mathrm{p}<0.01 ; * * *: \mathrm{p}<0.001 ; \mathrm{ns}$, not significant, as determined by two-tailed Student's $t$-tests. 


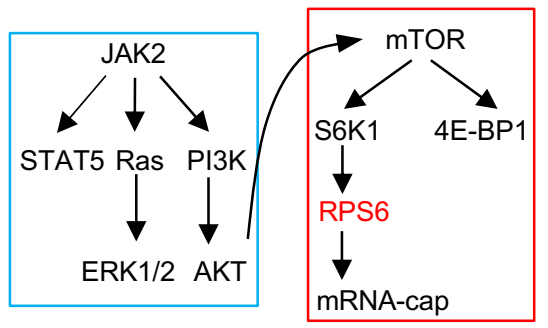

B

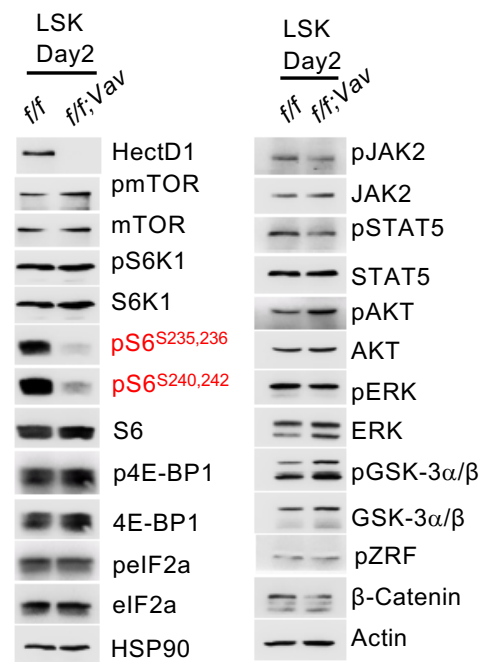

E

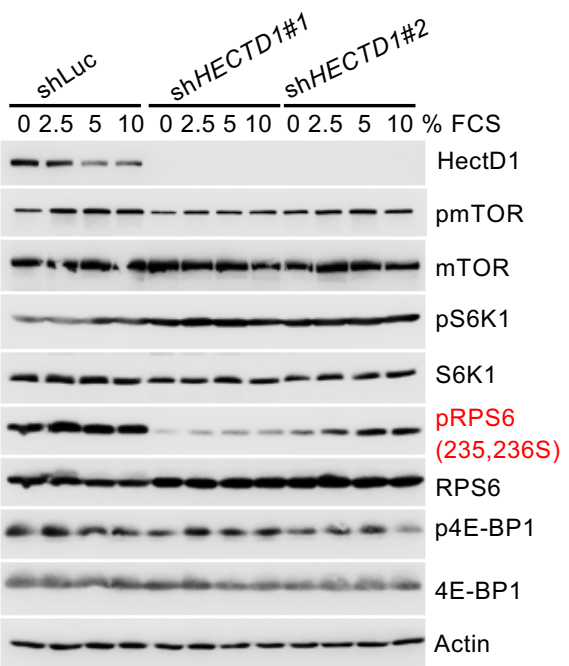
TPO $\frac{\text { ShLuC }}{0103060120} \frac{\text { ShHECTD1 }}{0103060120 \mathrm{~min}}$

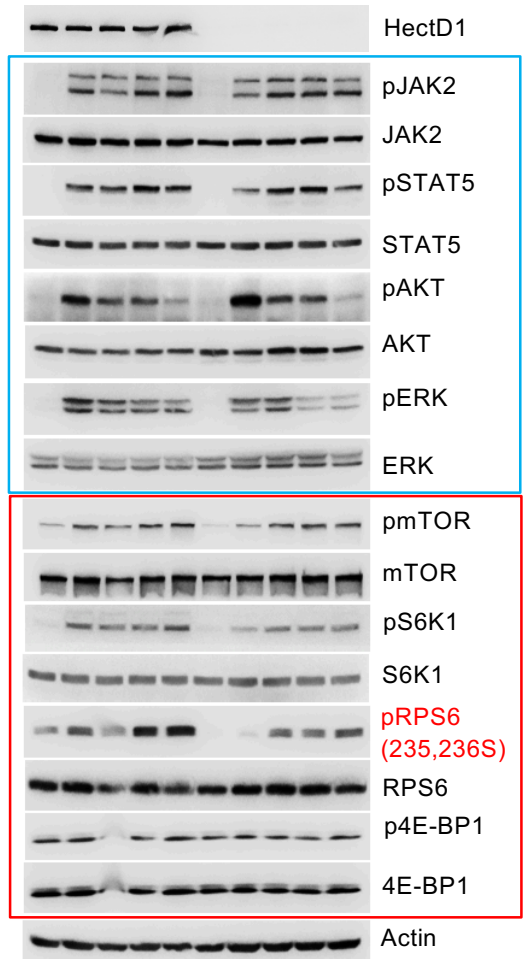

F

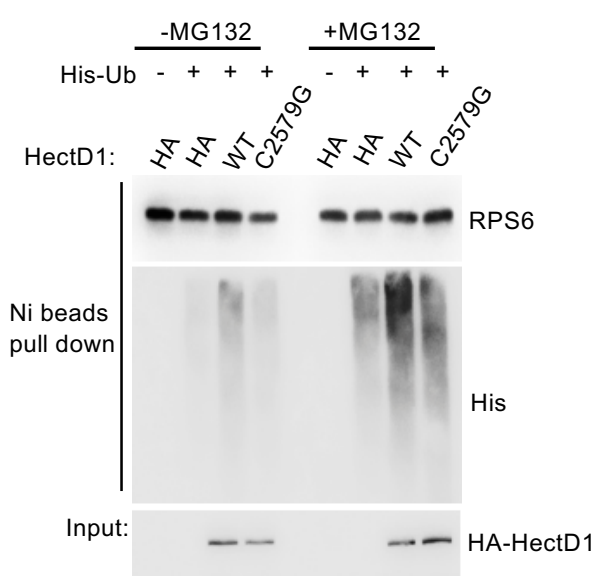
TPO $\frac{\text { shLuc }}{031030100031030100} \mathrm{ng} / \mathrm{mL}$

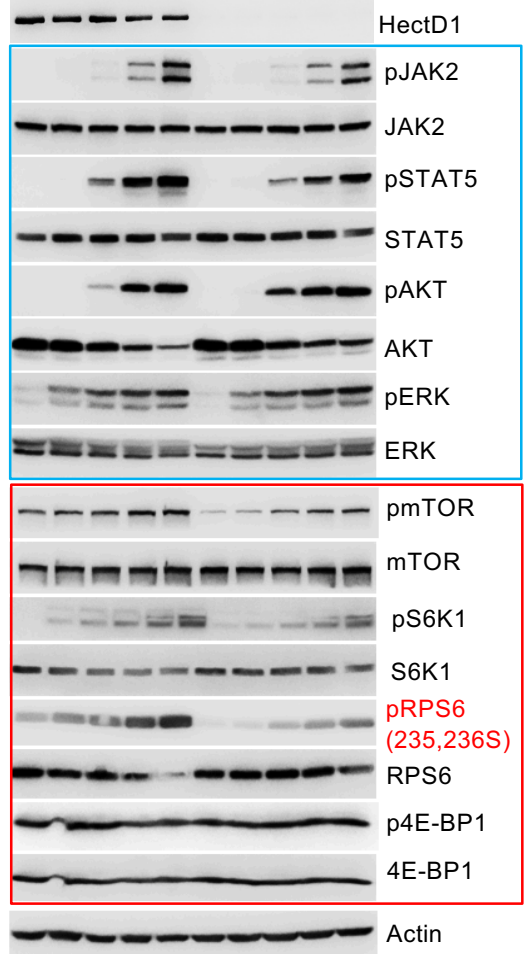

G

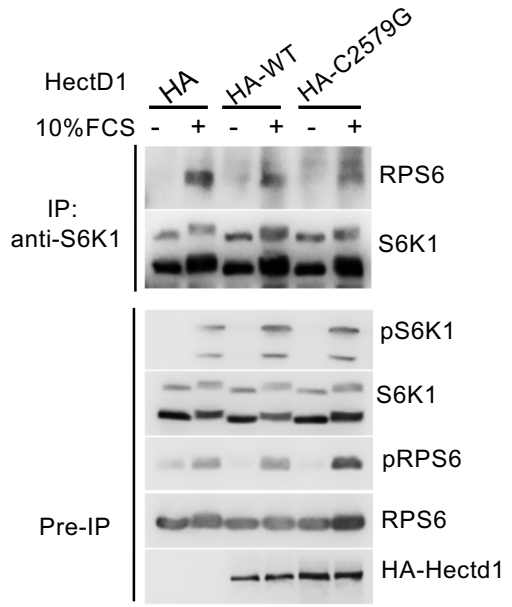

H

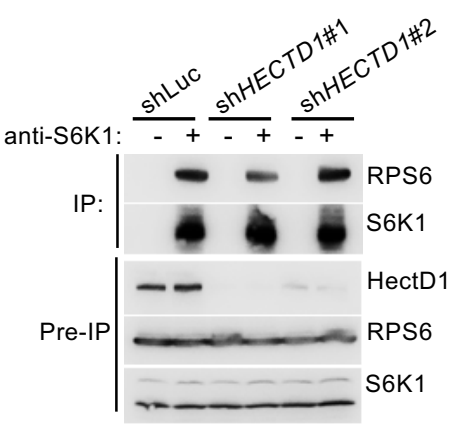

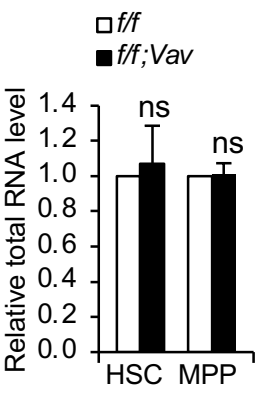

J

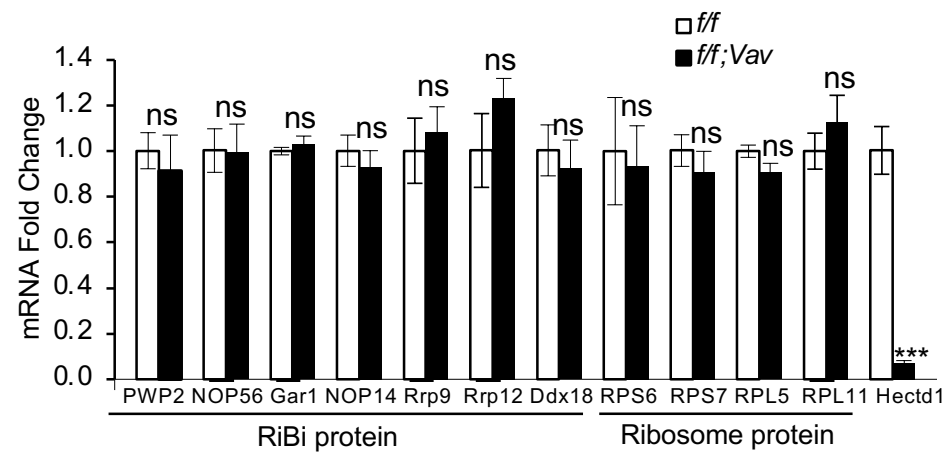


Figure S4. Hectd1 deficiency decreases RPS6 phosphorylation independent of the mTOR pathway. Related to figure 3.

(A-B) Hectd1 deficiency decreases RPS6 phosphorylation in 2 day-cultured LSK cells.

(A) A diagram showing different signaling pathways that we examined in LSKs and TF-1 cells.

(B) Two-day cultured LSKs from Hectd $1^{\text {fff }}$ and Hectd $1^{\text {ff;:Vav }}$ mice were used to examine various signaling molecules in the mTOR pathway, peIF2a, and JAK/STAT, PI3K-AKT, MAPK pathways by WB.

(C-E) HECTD1 depletion in TF-1 cells recapitulates the signaling properties observed in Hectd1-deficient LSK cells. (C-D) Signal kinetics (C) and sensitivity to cytokines (D) of JAK-STAT, PI3K-AKT, RAS-ERK and mTOR pathways were compared in shLuc and shHECTD1 TF-1/MPL cells stimulated with hTPO.

(E) Activation of the mTOR pathway was compared in shLuc vs shHECTDl cells upon stimulation of a graded concentration of fetal calf serum (FCS). Phospho-RPS6 (pRPS6) are highlighted in red.

(F-J) HectD1 does not affect RPS6 ubiquitination, S6K1-RPS6 interaction or the transcription of RiBi genes.

(F) 293T cells were transfected with constructs expressing HA control or HA-tagged HectD1 WT or HectdD1 E3-dead mutant C2579G. 48hrs later, cells were treated with or without proteasome inhibitor MG132 for 2hr, followed by His-Ub pull down under denatured conditions.

(G) 293 cells transfected with HA control or HA-tagged HectD1 WT or mutant C2579G were either untreated or deprived of serum. Activation of S6K1 and RPS6 by serum is shown in the WB (Pre-IP). S6K1-RPS6 interaction was assessed by S6K1 immunoprecipitation (IP) followed by WB with anti-RPS6 antibodies.

(H) HectD1 depletion does not affect S6K1-RPS6 interaction as determined by IP/WB in TF-1 cells.

(I) Total RNA was extracted and compared in HSCs and MPPs of Hectd ${ }^{f f f}$ and Hectd ${ }^{\text {ff; }}{ }^{\text {Vav }}$ mice.

(J) qRT-PCR analysis of the gene expression of RiBi proteins and ribosome proteins in HSCs of Hectd ${ }^{f f f}$ and Hectd ${ }^{\text {ff; Vav }}$ mice. $n=3$ in each group. 
A

\begin{tabular}{|c|c|c|c|c|}
\hline GO cellular component & $\begin{array}{l}\text { \# identified/ } \\
\text { in category }\end{array}$ & $\begin{array}{l}\text { Fold } \\
\text { enrichment }\end{array}$ & & FDR \\
\hline DNA replication factor $\mathrm{C}$ complex (GO:0005663) & $2 / 5$ & $>100$ & $3.18 \mathrm{E}-04$ & 1.49E-02 \\
\hline 3M complex (GO:1990393) & $2 / 5$ & $>100$ & $3.18 \mathrm{E}-04$ & $1.45 \mathrm{E}-02$ \\
\hline paraspeckles (GO:0042382) & $2 / 6$ & 84.32 & $4.22 \mathrm{E}-04$ & 1.77E-02 \\
\hline proteasome regulatory particle, lid subcomplex & & & & \\
\hline (GO:0008541) & $2 / 8$ & 63.24 & $6.75 E-04$ & 2.47E-02 \\
\hline $\begin{array}{l}\text { chaperonin-containing T-complex (GO:0005832) } \\
\text { eukaryotic translation initiation factor } 4 \mathrm{~F} \text { complex }\end{array}$ & $2 / 11$ & 45.99 & 1.16E-03 & 3.77E-02 \\
\hline (GO:0016281) & $2 / 13$ & 38.92 & $1.56 \mathrm{E}-03$ & 4.75E-02 \\
\hline exocyst (GO:0000145) & $3 / 20$ & 37.94 & 9.85E-05 & 6.01E-03 \\
\hline proteasome regulatory particle (GO:0005838) & $3 / 22$ & 34.5 & 1.27E-04 & 7.53E-03 \\
\hline proteasome accessory complex (GO:0022624) & $3 / 25$ & 30.36 & $1.80 \mathrm{E}-04$ & 9.77E-03 \\
\hline Flemming body (GO:0090543) & $3 / 31$ & 24.48 & $3.23 \mathrm{E}-04$ & 1.44E-02 \\
\hline cytosolic small ribosomal subunit (GO:0022627) & $4 / 47$ & 21.53 & 4.85E-05 & $3.25 \mathrm{E}-03$ \\
\hline cytoplasmic stress granule (GO:0010494) & $5 / 68$ & 18.6 & $1.02 \mathrm{E}-05$ & 8.56E-04 \\
\hline mitochondrial nucleoid (GO:0042645) & $3 / 44$ & 17.25 & 8.42E-04 & 2.97E-02 \\
\hline nucleoid (GO:0009295) & $3 / 44$ & 17.25 & 8.42E-04 & 2.92E-02 \\
\hline polysome (GO:0005844) & $5 / 75$ & 16.86 & $1.60 \mathrm{E}-05$ & 1.24E-03 \\
\hline proteasome complex (GO:0000502) & $4 / 66$ & 15.33 & $1.68 \mathrm{E}-04$ & 9.67E-03 \\
\hline endopeptidase complex (GO:1905369) & $4 / 67$ & 15.1 & $1.78 \mathrm{E}-04$ & 9.93E-03 \\
\hline ficolin-1-rich granule (GO:0101002) & $7 / 124$ & 14.28 & 8.54E-07 & 2.15E-04 \\
\hline ficolin-1-rich granule lumen (GO:1904813) & $7 / 4 / 124$ & 14.28 & 8.54E-07 & $1.91 \mathrm{E}-04$ \\
\hline small ribosomal subunit (GO:0015935) & $4 / 79$ & 12.81 & $3.24 \mathrm{E}-04$ & $1.42 \mathrm{E}-02$ \\
\hline cytosolic ribosome (GO:0022626) & $5 / 111$ & 11.39 & 9.54E-05 & $6.00 \mathrm{E}-03$ \\
\hline growth cone (GO:0030426) & $8 / 187$ & 10.82 & 1.01E-06 & 1.85E-04 \\
\hline peptidase complex (GO:1905368) & $4 / 94$ & 10.76 & $6.09 E-04$ & 2.41E-02 \\
\hline site of polarized growth (GO:0030427) & $8 / 195$ & 10.38 & 1.37E-06 & 2.30E-04 \\
\hline midbody (GO:0030496) & $7 / 182$ & 9.73 & $9.74 \mathrm{E}-06$ & 8.52E-04 \\
\hline nuclear matrix (GO:0016363) & $4 / 108$ & 9.37 & 1.00E-03 & 3.37E-02 \\
\hline ribosome (GO:0005840) & $8 / 247$ & 8.19 & 7.41E-06 & $6.78 \mathrm{E}-04$ \\
\hline $\begin{array}{l}\text { cytoplasmic ribonucleoprotein granule } \\
\text { (GO:0036464) }\end{array}$ & $7 / 224$ & 7.91 & $355 \mathrm{E}-05$ & $255 \mathrm{~F}-03$ \\
\hline ribonucleoprotein granule (GO:0035770) & $7 / 235$ & 7.54 & 4.77E-05 & 3.31E-03 \\
\hline secretory granule lumen (GO:0034774) & $9 / 320$ & 7.11 & $5.88 \mathrm{E}-06$ & 7.39E-04 \\
\hline cytoplasmic vesicle lumen (GO:0060205) & 9/324 & 7.03 & $6.48 \mathrm{E}-06$ & $6.52 \mathrm{E}-04$ \\
\hline vesicle lumen (GO:0031983) & $9 / 326$ & 6.98 & $6.80 \mathrm{E}-06$ & $6.52 \mathrm{E}-04$ \\
\hline distal axon (GO:0150034) & $8 / 307$ & 6.59 & 3.39E-05 & 2.53E-03 \\
\hline ribonucleoprotein complex (GO:1990904) & $18 / 709$ & 6.42 & $3.54 \mathrm{E}-10$ & 1.78E-07 \\
\hline ribosomal subunit (GO:0044391) & $5 / 197$ & 6.42 & $1.22 \mathrm{E}-03$ & 3.83E-02 \\
\hline
\end{tabular}

B

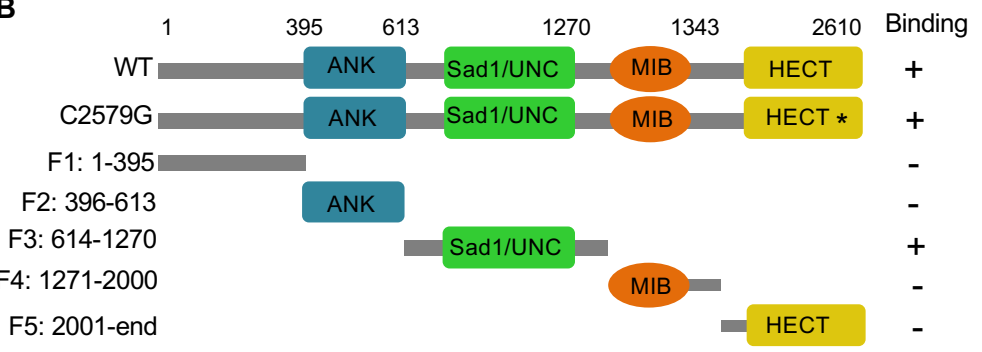

C

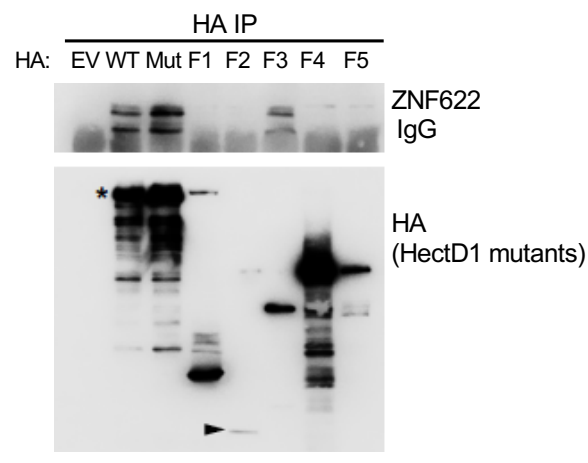

Input:

ZNF622

D

$\begin{array}{llllll}4 & 28 & 67 & 91 & 252 & 330\end{array} 477$ Binding

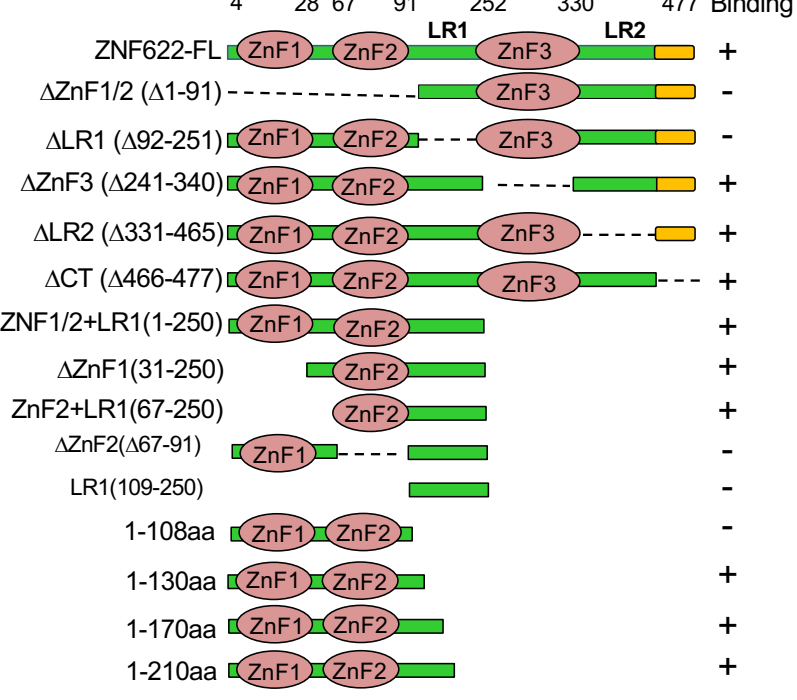

E

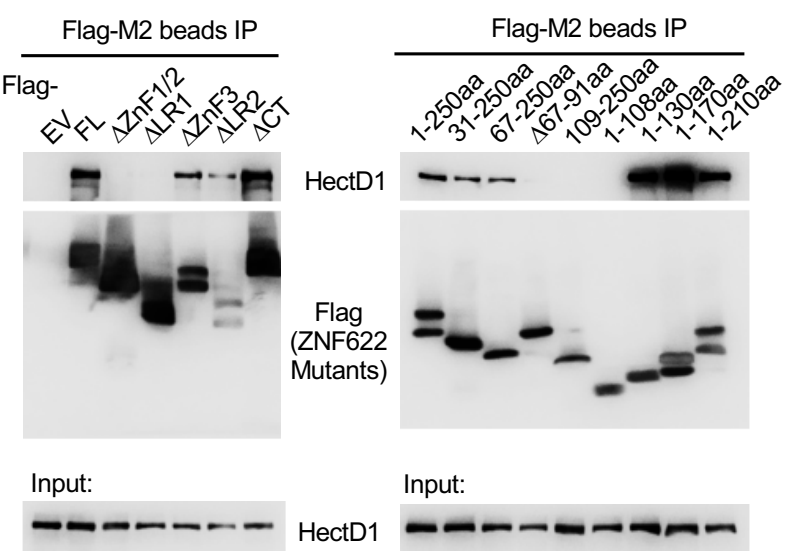

F

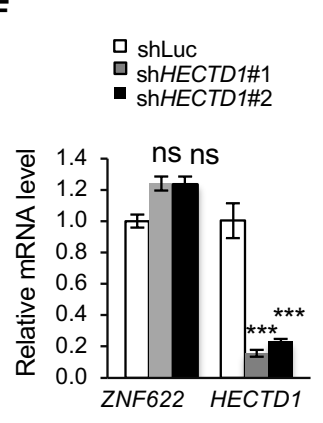

H

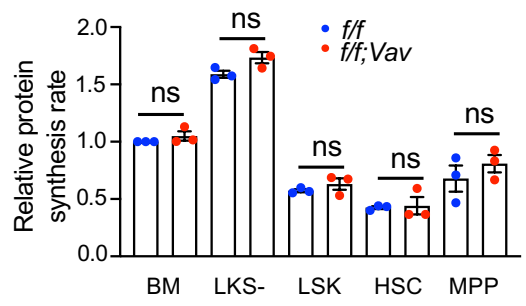

G

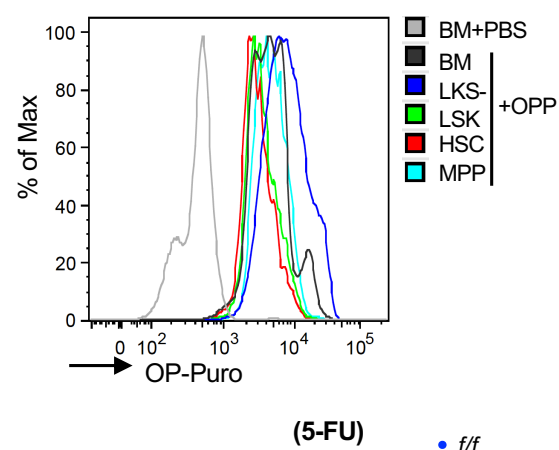

I

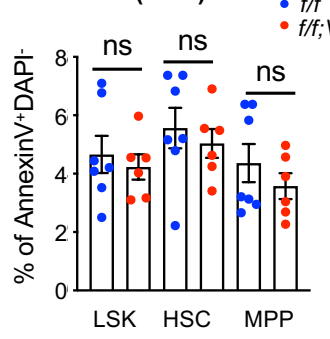


Figure S5. Panels S5A-S5F are related to figure3. Panels S5G-S5I, related to figure 4.

(A) Gene Ontology (GO) cellular component analysis of HectD1 interactors identified in three biological replicates of HAHectD1 immunoprecipitation/mass spectrometry (IP/MS). GO categories with fold enrichment $>5$ and FDR cutoff of 5\% are shown.

(B-E) Mapping the interacting domains/regions of HectD1 and ZNF622 by IP/WB.

(B) Schematic demonstration of HectD1 WT, E3-dead mutant (C2579G) and deletion mutants (Fragment1-5). Positive interaction is labeled with "+"; no interaction "-".

(C) HA-IP/WB in293T cells transfected with HA-tagged HectD1 WT or indicated mutants. Endogenous ZNF622 was immunoblotted. The full-length HectD1 band was marked by asterisk. Fragment 2 (F2) encompassing amino acid 396-613 was lowly expressed and marked by a solid arrow.

(D) Schematic demonstration of full-length ZNF622 and its deletion mutants. Positive interaction is labeled with "+"; no interaction "-".

(D) Flag-IP/WB in293T cells transfected with Flag-tagged ZNF622 FL or indicated truncates/mutants. Endogenous HectD1 was immunoblotted.

(F) qRT-PCR analysis of the mRNA level of ZNF622 in TF-1 cells with shLuc or shHECTD1\#1 and \#2. $\mathrm{n}=3$ in each group. Data are represented by mean \pm SD. ns, not significant; $* * *: \mathrm{p}<0.001$, as determined by unpaired two-tailed Student's $t$ tests.

(G-I) Both protein synthesis rate and cell apoptosis are not changed in primary Hectd1 ${ }^{\text {ff; Vav }}$ mice. $^{\text {(A) }}$

(A) Representative histogram of OP-Puro intensity in unfractionated BM cells and different subpopulations of stem and progenitor cells of a control mouse.

(B) Quantification of relative protein synthesis rate in unfractionated BM cells and different subpopulations of stem and progenitor cells of Hectd $1^{f / f}(\mathrm{n}=3)$ and Hectd $1^{\text {ff; Vav }}(\mathrm{n}=3)$ mice.

(C) Cell apoptosis analysis of LSKs, HSCs and MPPs in 5-FU challenged Hectd $1^{f f f}(\mathrm{n}=7)$ and Hectd1 ${ }^{\text {ff; Vav }}$ ( $\left.\mathrm{n}=6\right)$ mice by AnnexinV staining. Each symbol represents an individual mouse; bars indicate mean values; error bars indicate SE. ns: not significant, $\mathrm{p}>0.05$; as determined by two-tailed Student's $t$-tests. 
A

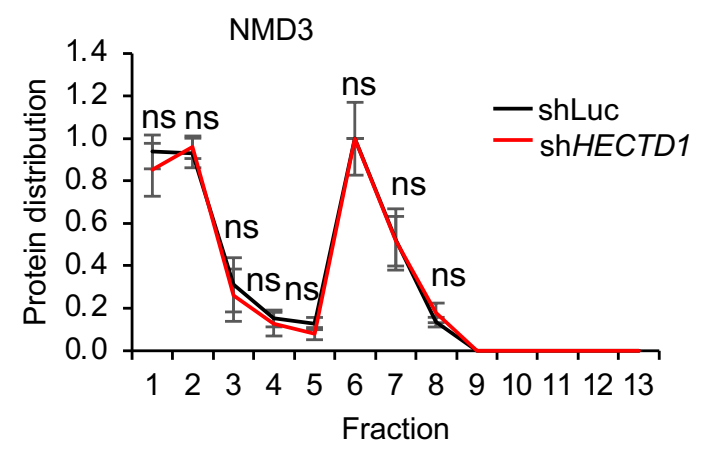

C

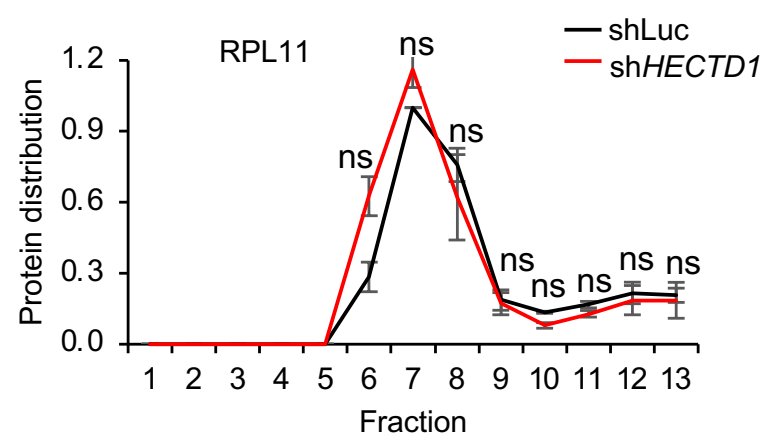

E

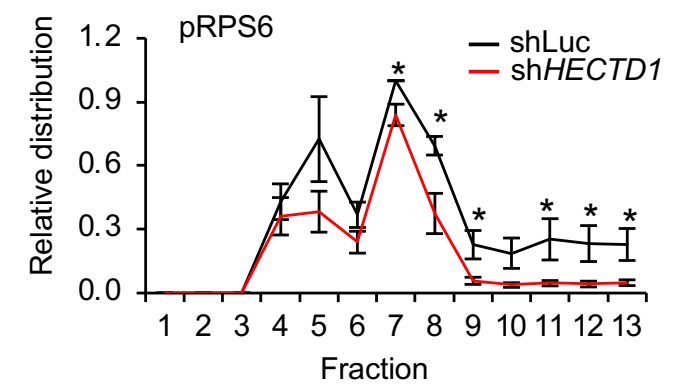

$\mathbf{F}$

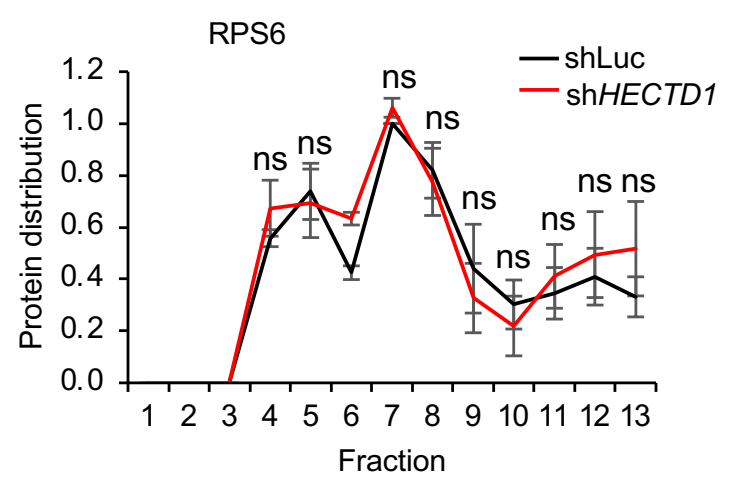

B

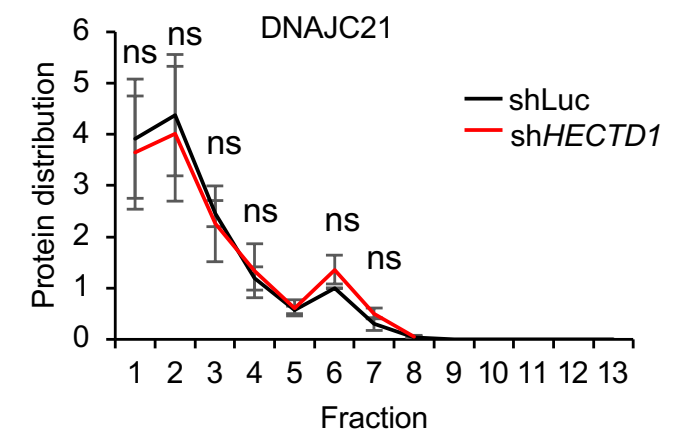

D

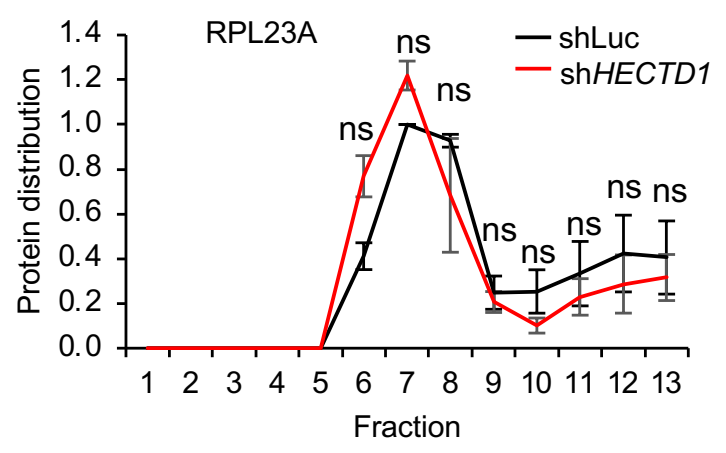

G

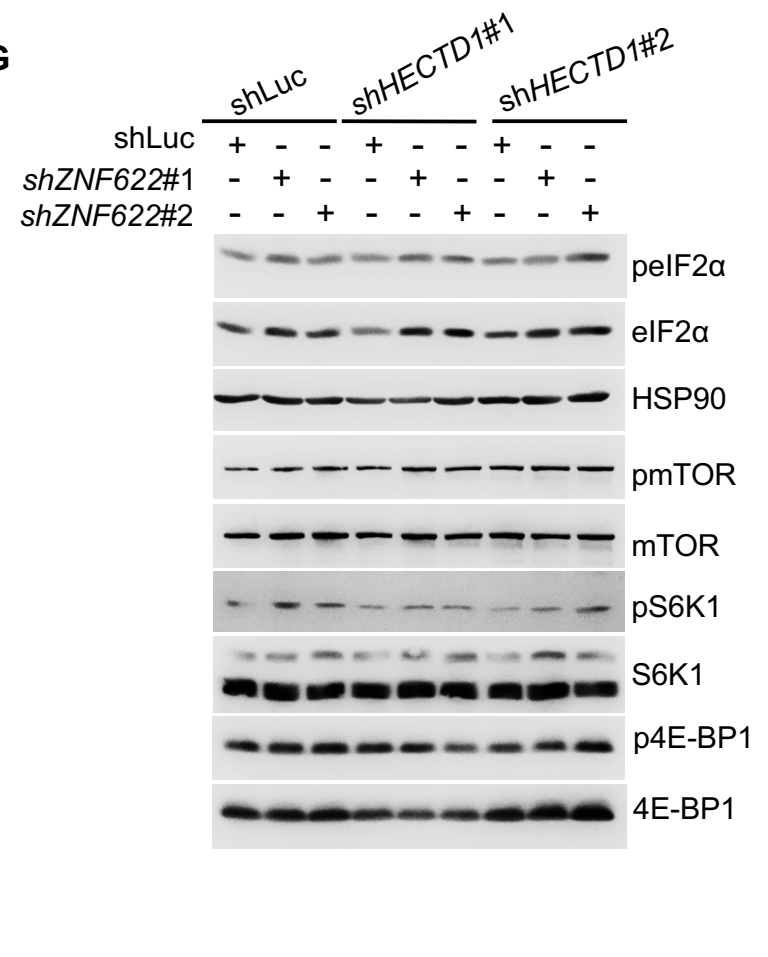

Figure S6. HECTD1 depletion reduces phospho-RPS6, but not NMD3 or select RPLs in ribosome fractions. Panels S6A-S6F are related to figure 5; S6G, related to figure 6.

(A-F) Quantification of the relative distribution of indicated proteins in three independent polysome profiling assays of shLuc and shHECTD1 TF-1 cells. Data are represented by mean \pm SE. $*$ : $\mathrm{p}<0.05$; ns, not significant, as determined by unpaired two-tailed Student's $t$-tests.

(G) Knockdown of ZNF622 does not change the phosphorylation of eIF2a or mTOR activation. WB of indicated proteins in TF-1 cells with single or double knockdown of HECTD1 and ZNF622. 


\title{
Click here to access/download Supplemental Videos and Spreadsheets
Tabel S1. HectD1 interactors by IP-MS. Related to
Figure 3.xlsx Supplemental Videos and Spreadsheets
Tabel S1. HectD1 interactors by IP-MS. Related to
Figure 3.xlsx \\ Supplemental Videos and Spreadsheets
Tabel S1. HectD1 interactors by IP-MS. Related to
Figure 3.xlsx
}

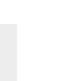

保 
Click here to access/download Supplemental Videos and Spreadsheets Table S2.Primers used are shown. Related to Star Methods.xlsx 